\title{
CONFORMAL DIMENSION VIA p-RESISTANCE: SIERPIŃSKI CARPET
}

\author{
Jaroslaw Kwapisz \\ Montana State University, Department of Mathematical Sciences \\ Bozeman MT 59717-2400, U.S.A.; jarek@math.montana.edu
}

\begin{abstract}
We put forth the notion of p-resistance as a proxy for the combinatorial p-modulus and demonstrate its effectiveness by studying the (Ahlfors regular) conformal dimension of the Sierpiński carpet. Specifically, we construct large resistor network approximating the carpet, establish weak-sup and sub-multiplicativity of their p-resistances, identify the conformal dimension as the associated critical exponent, and provide numerical approximations and rigorous two-sided bounds. In particular, we prove that the conformal dimension of the carpet exceeds $1+\ln 2 / \ln 3$, the Hausdorff dimension of the Cantor comb contained therein. A conjectural construction (and a numerical picture) of the quasi-symmetric uniformization of the carpet emerges as a byproduct.
\end{abstract}

\section{Introduction}

We give a mixture of theoretical and numerical results pertaining to the problem of finding the (Ahlfors regular) conformal dimension of the Sierpiński carpet, following the trailblazing numerical work in Malo's PhD thesis [26] and inspired by Barlow and Bass's treatment of the spectral dimension in [4]. Our overarching goal is to import into this context the concept of p-resistance and show that it is a fruitful proxy for the combinatorial p-modulus (or p-extremal length), which became a well established tool in this subject $[12,7,17,13,10]$. As will be explained, the basic qualitative results about p-resistance can be deduced from their p-modulus counterparts found in Carrasco Piaggio's [13] and Bourdon and Kleiner's [9]. However, the resistance based approach is quite effective for more delicate quantitative questions and facilitates radical speedup of numerical computations. It comes with two dualities (convex and topological) and lends itself to elementary yet powerful arguments based on combining and rearranging networks. To present the ideas in the simplest non-trivial case, we confine attention to the classical Sierpiński carpet, but such p-electrical engineering could be deployed in much greater generality, including the comprehensive setting in [13]. The ultimate hope is to apply our techniques to Cannon's conjecture, approached along the lines of [8].

The following introduction attempts a reasonably self-contained presentation of the main results and ideas. More detailed statements and proofs are relegated to sections 3 through 8 . We also include preliminary Section 2, to fix notations and review the principles for direct current (DC) circuits at (non-physical) exponent $\mathrm{p}>1$, not hitherto used in the context of conformal dimension. The many figures are an integral part of our results and arguments, and most should be viewed in color to be fully intelligible.

https://doi.org/10.5186/aasfm.2020.4515

2010 Mathematics Subject Classification: Primary 28A80, 30L10, 30C75, 31B15, 65 E05.

Key words: Ahlfors regular, conformal dimension, p-resistance, p-extremal length, quasisymmetric uniformization. 


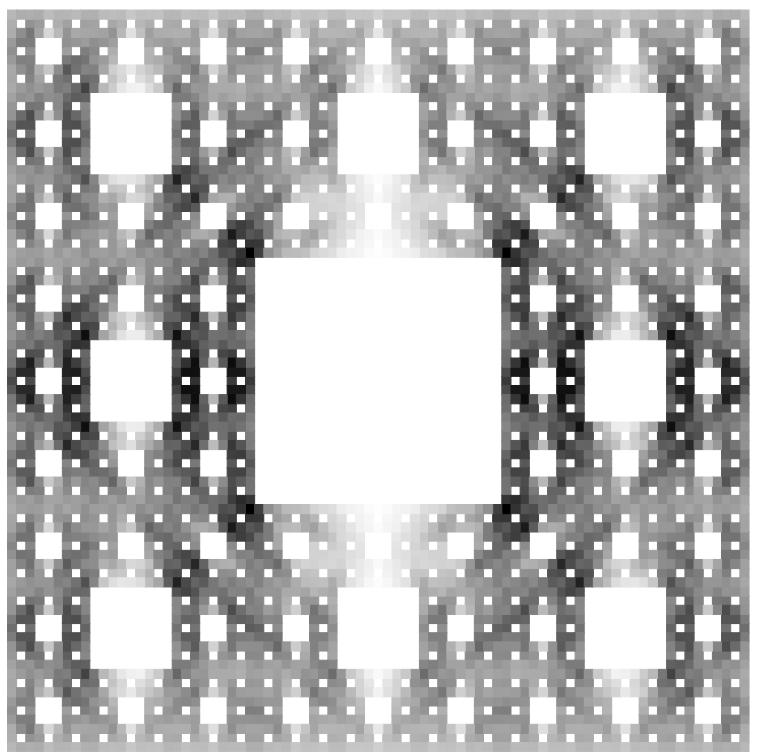

Figure 1.1. Sierpiński carpet approximant $X_{4}$, shaded by the approximate intensity of the $\mathrm{p}$-current flowing from the bottom to the top (for $\mathrm{p}=1.7965$ ).

1.1. Sierpiński carpet. Taking $\mathbf{N}_{0}:=\{0,1,2, \ldots\}$, the classical Sierpiński carpet $X$ (introduced in [30]) is the subset of the plane $\mathbf{R}^{2}$ given by the intersection $X=\bigcap_{n \in \mathbf{N}_{0}} X_{n}$ where $X_{0}=[0,1]^{2}$ is the unit square and the approximants $X_{n}$ are defined recursively by the rule that $X_{n+1}$ results from removing the (open) middle square of side length $3^{-n-1}$ from each of the $8^{n}$ squares making up $X_{n}$ (see Figure 1.1). $X$ is a metric space with the metric $d_{E}$ induced from the standard Euclidean distance on $\mathbf{R}^{2}$. It is infinitely ramified - cannot be disconnected by removing finitely many points - and, as such, provides a proving ground for a number of challenges in analysis on fractals (see e.g. [3]).

1.2. Conformal dimension. The Ahlfors regular conformal dimension of the carpet is the following infimum of the Hausdorff dimensions

$$
\operatorname{dim}_{A R}(X):=\left\{\operatorname{dim}_{H D}(X, d): d \sim_{q s} d_{E}\right\}
$$

where $d$ ranges over all Ahlfors regular metrics on $X$ and $\sim_{q s}$ denotes quasi-symmetric equivalence. The reader should consult [24, 18] for definitions and a discussion of the challenge presented by the dimension of the carpet. The simplicity of the construction of $X$ does not betray the difficulty of pinning down $\operatorname{dim}_{A R}(X)$. In that regard we obtain the following approximation:

\section{Numerical prediction:}

$$
\operatorname{dim}_{A R}(X) \approx 1.7965 .
$$

Strictly speaking, this is a prediction, an educated guess about the consecutive digits of $\operatorname{dim}_{A R}(X)$. It should be compared with the best effort to date found in Malo's thesis [26], where two digits of $\operatorname{dim}_{A R}(X)$ are predicted based on evidence suggesting that it sits between 1.7 and 1.8. While we have high confidence in at least the first four digits in 1.7965, we also provide rigorous computer assisted bounds (Sections 5 and 7 ), including the following:

\section{Rigorous computer assisted bounds:}

$$
1.765225 \leq \operatorname{dim}_{A R}(X) \leq 1.806703 .
$$


The above bounds (and the number 1.7965) were found by computations on the level $n=8$ approximant $X_{8}$, where a quasi-Newton iteration was used to solve a nonlinear system with roughly $10^{7}$ variables. Once a value of $\mathrm{p}$ is fixed, the proof of a bound (by this $\mathrm{p}$ ) amounts to a simple act of evaluating the p-norm of the solution. With more computing effort, the spread between the upper and lower bounds could be narrowed; in particular, a lower bound by 1.783499 is readily achievable (Proposition 7.8), but we had to stop somewhere. We give details on the numerical procedures used in Section 8.

Effectiveness of our methods is not solely due to the computing power deployed. To demonstrate this point (and bow to the traditional standards of mathematical discourse), we state below much weaker bounds obtained in the simplest case, when $n=1$ and hand computation is feasible.

Theorem 1.1.

$$
1.707491 \leq \operatorname{dim}_{A R}(X) \leq 1.847596
$$

This already improves on the previous state of art:

$$
1.6309 \approx 1+\frac{\ln 2}{\ln 3} \leq \operatorname{dim}_{A R}(X) \leq \frac{\ln ((9+\sqrt{41}) / 2))}{\ln 3} \approx 1.858,
$$

due to Tyson [31] and Kigami [21], respectively. Incidentally, Tyson's lower bound comes from $X$ containing the Cantor comb, the product of the middle-thirds-Cantor set and segment $[0,1]$. Its sharpness has been an open question for some time (see, e.g., Problem 15.22 in [18]). Conceptually, our affirmative answer rests on rigorous expression of the intuition that the top-to-bottom current in $X$ has to spill beyond the comb and utilize more of the carpet, as illustrated by Figure 1.1 (see also Figure 7.20).

Note: In his new work [22], Kigami constructed an even more coprehensive version of Carassco Piagio's [13] framework with p-modulus replaced by p-energy, which is equivalent to the p-resistance defined via potentials (as in Propositon 2.3). As an application, he showed that $\operatorname{dim}_{A R}(X)$ is boundeed above by the spectral dimension studied by Barlow and Bass. For the Sierpiński carpet, [5] predicted the spectral dimension to be approximately 1.8052 by computing up to the level $n=7$. This is better then our rigorous numerical upper bound 1.8067 obtained for $n=8$ (cf. Proposition 5.4). However, unlike 1.8067, the number 1.8052 is derived by Shank's transform based extrapolation - much like our prediction 1.7965 . On the flip side, the numerical problem being linear for $\mathrm{p}=2$, the spectral dimension could be further approximated by using modern computers to levels $n=8$ or 9 . Additionally, in [2], Albin et al. develop a theory of p-modulus for families of paths on graphs, including the requisite convex duality and a discussion of applications and open problems. Note that they use the edge-weighted version of the p-modulus, which coincides with the p-conductance (the reciprocal of the p-resistance), but is not quite the same as the vertex-weighted p-modulus we borrowed from [13, 26].

1.3. Uniformization picture. While chasing the digits of $\operatorname{dim}_{A R}(X)$ may not seem like a worthwhile pursuit, it sheds light on the existence of a metric realizing the infimum in the definition of $\operatorname{dim}_{A R}(X)$, a problem of great interest in connection with Kapovich-Kleiner Conjecture [20,6]. We propose one concrete way of generating such an optimal metric by constructing a sequence of maps $\left(F_{n}\right)_{n \in \mathbf{N}}$ defined on graph approximations $\mathcal{G}_{n}$ to $X$ (depicted in Figure 1.3 and detailed in subsection 1.4, ahead). The conjecture is that $F_{n}$ converge (in the Gromov-Hausdorff sense) to a quasisymmetric homeomorphism $F: X \rightarrow X_{\text {slit }}$ where $X_{\text {slit }}$ is a planar slit carpet, a subset 
obtained by making infinitely many vertical slits in the interior of a rectangle in $\mathbf{R}^{2}$ (see [25]). In such case, the optimal metric $d$ would coincide with the pull back via $F$ of the natural path metric on $X_{\text {slit }}$, and $F$ could be viewed as a quasi-symmetric uniformization of Sierpiński carpet. While $F_{n}$ are constructed in subsection 1.9, after necessary preliminaries are gathered, the reader is invited now to ponder the depiction of $F_{5}$ in Figure 1.2. This is how Sierpiński carpet (approximately) should look to a complex analyst. ( $F_{6}$ offers too much detail to render on a printed page.)

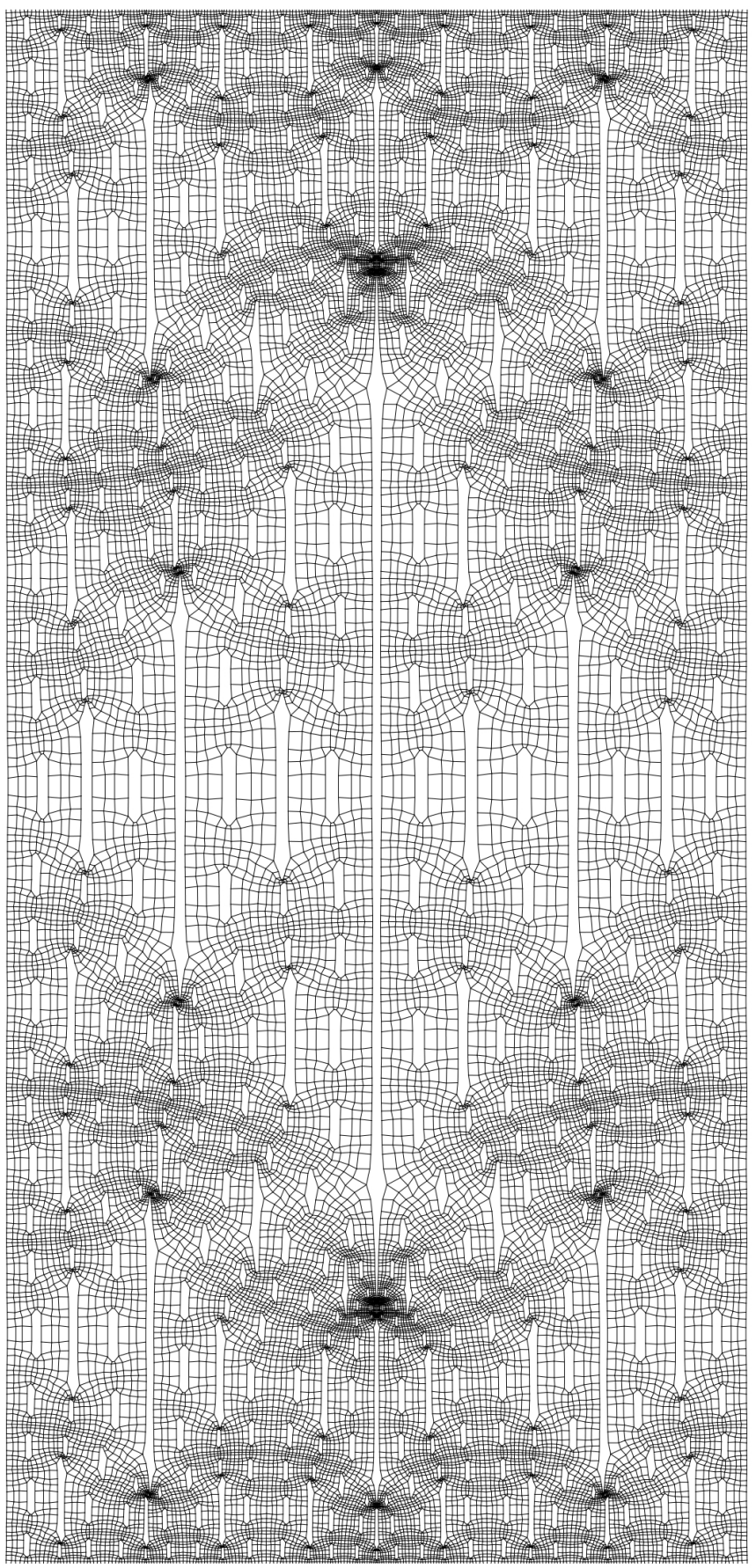

Figure 1.2. Slit carpet uniformizing $X$ (at approximation level $n=5$ ). 
1.4. p-Resistance. Our approach to $\operatorname{dim}_{A R}(X)$ is inspired by [4] where Barlow and Bass study the spectral dimension of $X$ by using the ordinary electrical resistance of its approximations. We simply replace the classical DC circuit theory by its counterpart at a variable exponent $\mathrm{p}>1$, as is done by substituting the $L^{\mathrm{p}}$-norm in the place of the $L^{2}$-norm representing the energy dissipated by the current. This is of course an old exploit used for a variety of theoretical and practical pursuits, particularly underlying the standard theory of $\mathrm{p}$-harmonic functions and the $\mathrm{p}$-Laplacian (see e.g. [16, 23, 11, 1, 19]). In the theory of conformal dimension, it offers an alternative to the methods based on the p-modulus or p-extremal length. Specifically, we study the p-resistance across an approximation to $X$ presented as a resistor network, a suitable finite graph with non-negative weights (resistances) over the edges. The most straightforward approximations, much like the standard graph approximations in [26], are the graphs $\mathcal{G}_{n}=\left(\mathcal{V}_{n}, \mathcal{E}_{n}\right)$ where the vertex set $\mathcal{V}_{n}$ consists of the vertices of the squares making up the $X_{n}$ and the edge set $\mathcal{E}_{n}$ collects the sides of those squares. (We work with oriented graphs but the p-resistance does not depend on the orientation, so we often suppress it, as is done in Figure 1.3.) For visual appeal, we chose not to include in $\mathcal{G}_{n}$ the horizontal edges along the top and bottom of $X_{n}$. (Those edges will carry no current a priori.) The resistance of an edge $e \in \mathcal{E}_{n}$ is $r(e)=\frac{1}{2}$, unless $e$ lies in the boundary of $X_{n}$, in which case $r(e)=1$. We note that the values of the resistances of the individual edges in $\mathcal{E}_{n}$ are immaterial for the determination of $\operatorname{dim}_{A R}(X)$, as long as they are uniformly bounded from 0 and $\infty$ (Theorem 3.1). The specific resistance choices we made are dictated by the recursive rules generating the networks $\mathcal{G}_{n}$ (in Section 6), which influence our approximations to $\operatorname{dim}_{A R}(X)$.

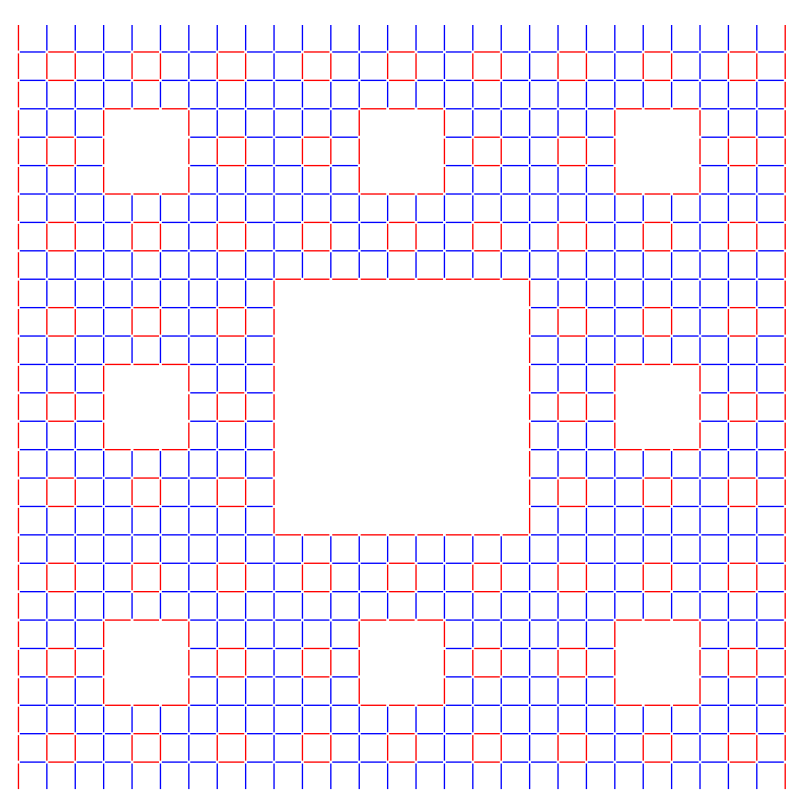

Figure 1.3. Standard network $\mathcal{G}_{3}$ : edge resistance is $r(e)=\frac{1}{2}$ for blue and $r(e)=1$ for red edges.

Denoting by $\mathcal{A}_{n}$ and $\mathcal{B}_{n}$ the sets of top and bottom vertices, respectively, we consider flows from $\mathcal{A}_{n}$ to $\mathcal{B}_{n}$; namely, functions $\mathcal{J}: \mathcal{E}_{n} \rightarrow \mathbf{R}$ that satisfy Kirchhoff's first law, i.e., have vanishing divergence, $\operatorname{div} \mathcal{J}(v)=0$, at each vertex $v \in \mathcal{V}_{n} \backslash$ $\left(\mathcal{A}_{n} \cup \mathcal{B}_{n}\right)$. (Detailed definitions are given in Section 2.) The flux of $\mathcal{J}$ is $J(\mathcal{J}):=$ 
$\sum_{v \in \mathcal{B}_{n}} \operatorname{div} \mathcal{J}(v)=\sum_{v \in \mathcal{A}_{n}}-\operatorname{div} \mathcal{J}(v)$, and its p-power is

$$
P(\mathcal{J}):=\sum_{e \in \mathcal{E}_{n}} r(e)^{\mathrm{p}^{*} / \mathrm{p}}|\mathcal{J}(e)|^{\mathrm{p}^{*}}
$$

where $\mathrm{p}^{*}$ is the Hölder exponent dual to $\mathrm{p}$, i.e., $1 / \mathrm{p}+1 / \mathrm{p}^{*}=1$. The $\mathrm{p}$-resistance from $\mathcal{A}_{n}$ to $\mathcal{B}_{n}$ is the result of a minimization over all flows from $\mathcal{A}_{n}$ to $\mathcal{B}_{n}$ :

$$
R_{n}(\mathrm{p}):=\min _{\mathcal{J}} \frac{P(\mathcal{J})^{\mathrm{p} / \mathrm{p}^{*}}}{J(\mathcal{J})^{\mathrm{p}}}
$$

where (by homogeneity) $J_{0}:=J(\mathcal{J})$ can be set to any non-zero value, say $J_{0}=1$. The function $\mathcal{J} \mapsto P(\mathcal{J})$ is strictly convex on the finite dimensional affine space of unit flux flows $\mathcal{J}$, and the minimum is uniquely realized. We refer to the optimal flow as a p-current (or simply current when it is clear what $\mathrm{p}$ is used) and call (1.3) a current power minimization problem.

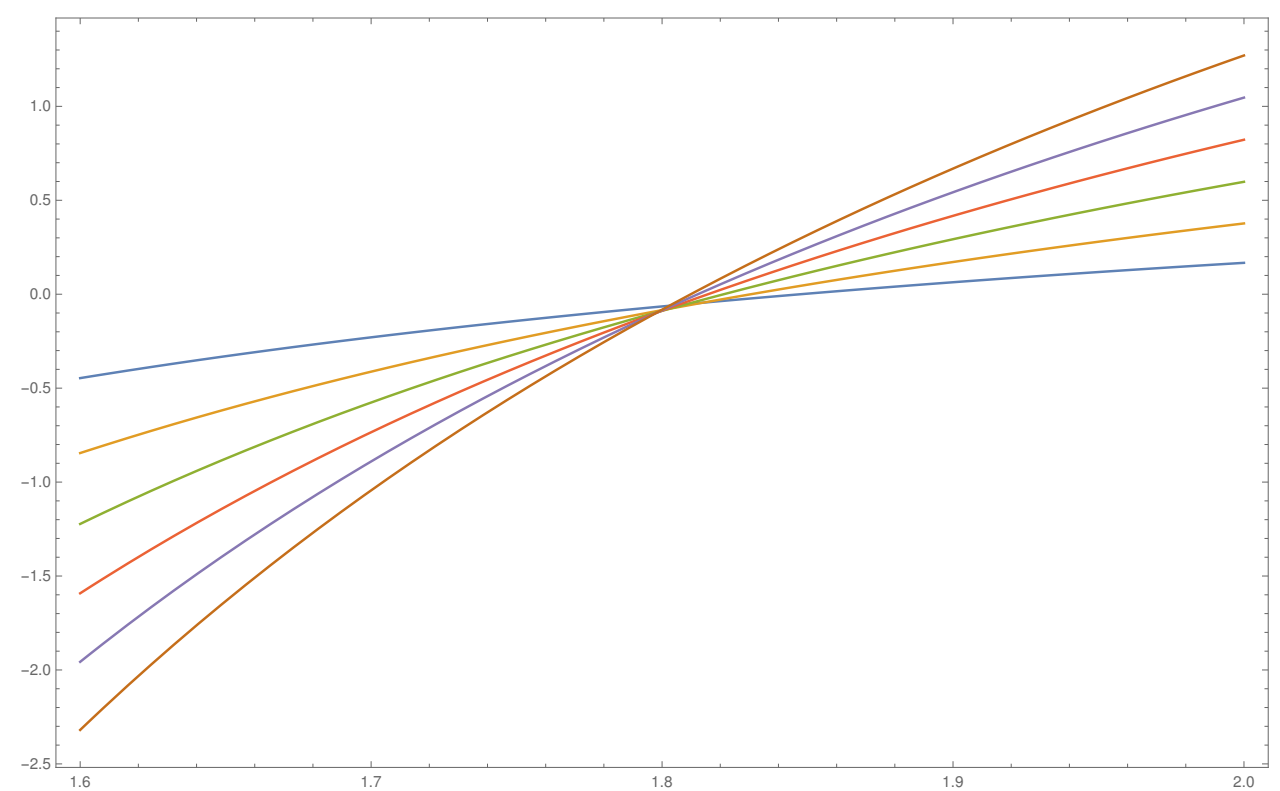

Figure 1.4. Log of the power ratio $\ln \rho_{n}(\mathrm{p})=\frac{\mathrm{p}^{*}}{\mathrm{p}} \ln \left(\frac{R_{n}(\mathrm{p})}{1 / 2}\right)$ as a function of $\mathrm{p}$, steepening with increasing $n$ (for $n=1, \ldots, 6)$.

We computed the current with flux $J_{0}=2$ through $\mathcal{G}_{n}$ and its power $P_{n}$ for $n \leq 8$ (Section 8). For $n=0, \mathcal{J}(e)=1$ on the two parallel resistors constituting $\mathcal{G}_{0}$, so $P_{0}=2$ and $R_{0}(\mathrm{p})=1 / 2$. Figure 1.4 shows plots ${ }^{1}$ of the logarithm of the power ratio

$$
\rho_{n}(\mathrm{p}):=\frac{P_{n}}{P_{0}}=\left(\frac{R_{n}(\mathrm{p})}{R_{0}(\mathrm{p})}\right)^{\frac{\mathrm{p}^{*}}{\mathrm{p}}} .
$$

We depicted $\ln \rho_{n}(\mathrm{p})$, in lieu of $R_{n}(\mathrm{p})$, because that is the quantity that will serve best to articulate our estimates. In particular, the zeros of $\ln \rho_{n}(\mathrm{p})$ will give the upper bounds (in Section 5). The plots of $\ln R_{n}(\mathrm{p})$ are similar.

1.5. Critical exponent. The gateway to our approximation to $\operatorname{dim}_{A R}(X)$ is identification of this dimension as the critical exponent for the p-resistance:

\footnotetext{
${ }^{1}$ Due to the numerical cost, for $n=7,8$, we used a narrower range of $\mathrm{p}$ and the corresponding plots are not included in Figure 1.4.
} 
Theorem 1.2. The Ahlfors-regular conformal dimension $\operatorname{dim}_{A R}(X)$ coincides with the resistance dimension of $X$, defined as

$$
\mathrm{p}_{\text {res }}:=\inf \left\{\mathrm{p}>1: \limsup _{n \rightarrow \infty} R_{n}(\mathrm{p})=\infty\right\} .
$$

We note that, a priori, the set of $\mathrm{p}$ under the inf above is either one of $\left(\mathrm{p}_{\mathrm{res}}, \infty\right)$ or $\left[\mathrm{p}_{\mathrm{res}}, \infty\right)$ (due to monotonicity of $\mid \mathcal{J}(e) \mathrm{p}^{\mathrm{p}}$ as a function of $\mathrm{p}^{*}$; cf. Corollary 1.4). The actual behavior of $R_{n}(\mathrm{p})$ is revealed by the plots in Figure 1.4, suggestively pivoting about a single point and tending to $\pm \infty$ on its opposite sides with the increasing of $n$ (although, zoomed Figure 8.25 reveals more nuance). Malo [26] uses the analogous characterization based on the combinatorial p-modulus and derived from a sweeping result, Theorem 1.3 in Carrasco Piaggio's [13] — itself rooted in Pansu's approach to the dimension in [27] and preceded by unpublished work of Keith and Kleiner (see remarks after Theorem 1.2 in [13] and at the end of sec. 3 in [9]). In particular, to prove the theorem one only has to verify that the p-resistance is comparable to the p-modulus, or rather to its reciprocal, the (combinatorial) extremal p-length (defined in subsection 1.6).

While Theorem 1.2 is a manifestation of a general principle, it is the self-similarity that affords the following result, which is also the departure point for our rigorous estimates of $\operatorname{dim}_{A R}(X)$.

Theorem 1.3. The sequence $\left(R_{n}(\mathrm{p})\right)_{n \in \mathbf{N}}$ is weakly sub- and sup-multiplicative, i.e., there are $\alpha(\mathrm{p}), \beta(\mathrm{p})>0$ (explicitly given and continuous in $\mathrm{p}$ ) so that, for $m, n \in \mathbf{N}$,

$$
\alpha(\mathrm{p})^{-\mathrm{p}} R_{n}(\mathrm{p}) R_{m}(\mathrm{p}) \leq R_{n+m}(\mathrm{p}) \leq \beta(\mathrm{p})^{\mathrm{p}} R_{n}(\mathrm{p}) R_{m}(\mathrm{p}) .
$$

For $\mathrm{p}=2$, the inequalities of Theorem 1.3 follow from those in [4], and the general case is similar; although, we use different networks and have to accommodate the lack of Hilbert space structure on $L^{\mathrm{p}}$. In a nutshell, weak sub-multiplicativity arises from gluing smaller networks to form bigger ones. Weak sup-multiplicativity is in the same vein once viewed on the other side of the topological duality of planar networks (which we discuss in subsection 1.8). We note that $[9,13]$ already show $^{2}$ sup-multiplicativity for extremal p-length in a general approximately self-similar setting, and Lemma 4.4 in [9] also tackles sub-multiplicativity for the specific case of the Sierpiński carpet. Thus Theorem 1.3 could be derived by simply using that the extremal p-length and the p-resistance are comparable (Theorem 3.1). However, beyond giving an alternative proof, we offer constructions of reasonably small constants $\alpha(\mathrm{p})$ and $\beta(\mathrm{p})$. E.g., beside the more involved formulas (4.15) and (6.5) valid for all $p>1$, for $p \in(1,2)$ - which is the range of primary interest - we are able to use Clarkson's inequality to obtain $\alpha(\mathrm{p})=2^{-1 / \mathrm{p}}$ (Corollary 5.3 ); and also $\beta(p)=2^{3 / \mathrm{p}-2}$ (Corollary 7.5), albeit the latter is for an alternative sequence of approximating networks, as discussed below. (For the original network see formula (6.7).) Naturally, one has to pay attention to what families of curves or networks are considered, as careful choices yield better constants.

Concerning $\operatorname{dim}_{A R}(X)$, Theorem 1.3 can be applied as follows: checking that $\beta(\mathrm{p})^{\mathrm{p}} R_{m}(\mathrm{p})<1$ for a specific $\mathrm{p}$ guarantees that $R_{n}(\mathrm{p})$ converges geometrically to 0 (as $n \rightarrow \infty$ ) and thus gives a lower bound, $\mathrm{p}_{\text {res }} \geq \mathrm{p}$. Similarly $\alpha(\mathrm{p})^{-\mathrm{p}} R_{m}(\mathrm{p})>1$ yields an upper bound $\mathrm{p}_{\text {res }} \leq \mathrm{p}$. Although $\alpha(\mathrm{p})$ and $\beta(\mathrm{p})$ are computed explicitly, the

\footnotetext{
${ }^{2}$ Their results are phrased in terms of the p-modulus, the reciprocal of the extremal p-lengthwhich switches "sub-" and "sup-".
} 
resulting bounds on $\mathrm{p}_{\text {res }}$ are not the best and can be improved by verifying weaker conditions: $\sup _{n} R_{n+m}(\mathrm{p}) / R_{n}(\mathrm{p})<1$ and $\inf _{n} R_{n+m}(\mathrm{p}) / R_{n}(\mathrm{p})>1$, respectively, for a specific value of $m$. (We used $m=1, \ldots, 8$.) This is the technical heart of this paper and not without some important nuance, which we would like to address now, even before all the definitions are in place.

For the lower bound, the naive idea is to use that $\mathcal{G}_{n+m}$ can be glued from copies of $\mathcal{G}_{m}$ and deduce $\sup _{n} R_{n+m}(\mathrm{p}) / R_{n}(\mathrm{p})<1$ from $R_{m}(\mathrm{p}) / R_{0}(\mathrm{p})<1$, which is equivalent to the power ratio bound $\rho_{m}(\mathrm{p})<1$. However, gluing flows on $\mathcal{G}_{m}$ into one flow on the bigger network $\mathcal{G}_{n+m}$ is impossible and one is lead to use alternative networks $\tilde{\mathcal{G}}_{n}$ (see Figure 7.22) with resistances comparable to those of $\mathcal{G}_{n}$. In particular, the power ratio relevant for the lower bound is not that plotted in Figure 1.4; see Figure 7.23 instead.

For the upper bound, we pass to the topologically dual network $\mathcal{G}_{n}^{*}$ (see Figure 1.6), in order to trade $\inf _{n} R_{n+m}(\mathrm{p}) / R_{n}(\mathrm{p})>1$ for $\sup _{n} R_{n+m}^{*}(\mathrm{p}) / R_{n}^{*}(\mathrm{p})<1$ and then mimic what we did for the lower bound. Luckily, the gluing of flows in the dual network $\mathcal{G}_{n}^{*}$ is easy and readily reduces the problem to a power ratio bound. Keep in mind that this power ratio bound is on the other side of the topological duality and the requisite inequality will translate (via (1.21)) to $\rho_{m}(\mathrm{p})>1$ in terms of the power ratio of the original network $\mathcal{G}_{n}$ (as plotted in Figure 1.4). Overall, the sup-multiplicativity and the upper bounds are technically easier and thus we give their exposition first (Sections 4 and 5), with the sub-multiplicativity and the lower bounds to follow (Sections 6 and 7 ).

Before proceeding with a more detailed exposition, we record the following corollary of Theorem 1.3.

Corollary 1.4. At $\mathrm{p}=\mathrm{p}_{\mathrm{res}}, R_{X}^{-}:=\liminf _{n \rightarrow \infty} R_{n}(\mathrm{p})$ and $R_{X}^{+}:=\limsup _{n \rightarrow \infty} R_{n}(\mathrm{p})$ satisfy

$$
\beta(\mathrm{p})^{-\mathrm{p}} \leq R_{X}^{-} \leq R_{X}^{+} \leq \alpha(\mathrm{p})^{\mathrm{p}} .
$$

For $\mathrm{p} \neq \mathrm{p}_{\text {res }}$, the limit $R_{X}(\mathrm{p}):=\lim _{n \rightarrow \infty} R_{n}(\mathrm{p})$ exists, and it equals 0 for $\mathrm{p}<\mathrm{p}_{\text {res }}$ and $\infty$ for $\mathrm{p}>$ pres.

Proof. Note that $R_{m}(\mathrm{p}) \leq \alpha(\mathrm{p})^{\mathrm{p}}$ for $\mathrm{p}=\mathrm{p}_{\text {res }}$ because, should it fail (for some $m \in$ $\mathrm{N})$, then $\alpha(\mathrm{p})^{-\mathrm{p}} R_{m}(\mathrm{p})>1$ and $\lim _{n \rightarrow \infty} R_{n}(\mathrm{p})=0$ for all $\mathrm{p}$ near $\mathrm{p}_{\text {res }}$, contradicting the definition of $\mathrm{p}_{\text {res }}$. Consequently, $R_{X}^{+} \leq \alpha\left(\mathrm{p}_{\mathrm{res}}\right)^{\mathrm{p}_{\mathrm{res}}}$. The rest of the proof is left for the reader.

The question whether the limiting resistance $R_{X}:=R_{X}\left(\mathrm{p}_{\mathrm{res}}\right)$ is well defined is open and constitutes a stepping stone toward the existence of the extremal metric.

1.6. Resistance vs extremal length. Our next main point is the numerical superiority of p-resistance over the combinatorial extremal p-length, as revealed below by a brief look at the latter.

Write $\Gamma_{n}$ for the collection of vertex sets of all paths connecting $\mathcal{A}_{n}$ to $\mathcal{B}_{n}$ in $\mathcal{G}_{n}$ (treated as an undirected graph). The (discrete) extremal p-length of the family $\Gamma_{n}$ is defined as

$$
\Lambda_{n}(\mathrm{p}):=\max _{\mathrm{m}} \frac{\left(\min _{\gamma \in \Gamma_{n}} \sum_{v \in \gamma} \mathrm{m}(v)\right)^{\mathrm{p}}}{\sum_{v \in \mathcal{V}_{n}} \mathrm{~m}(v)^{\mathrm{p}}}
$$

where the maximum is taken over all non-zero functions $\mathrm{m}: \mathcal{V}_{n} \rightarrow[0, \infty)$, called weights. For most purposes $\Lambda_{n}(\mathrm{p})$ is interchangeable with $R_{n}(\mathrm{p})$ because the two are comparable: we show that there is $C>0$, independent of $n$ and $\mathrm{p}$, such that 
$C^{-1} R_{n}(\mathrm{p}) \leq \Lambda_{n}(\mathrm{p}) \leq C R_{n}(\mathrm{p})$ (see Section 3). In particular, $\Lambda_{n}(\mathrm{p})$ exhibit the same type of critical behavior as $R_{n}(\mathrm{p})$, with a critical jump from 0 to $+\infty$ at

$$
\mathrm{p}_{\mathrm{res}}=\mathrm{p}_{\mathrm{el}}:=\inf \left\{\mathrm{p}>1: \limsup _{n \rightarrow \infty} \Lambda_{n}(\mathrm{p})=+\infty\right\}
$$

As already mentioned, the equality $\mathrm{p}_{\mathrm{el}}=\operatorname{dim}_{A R}(X)$ was shown in [26] based on its variant in [13] where, roughly speaking, all paths with definite diameter are considered.

Compare the overall structures of (1.6) and (1.3). The max-min nature of (1.6) makes it more complicated but some of this complexity can be removed. By scaling, one restricts to $\mathrm{m}$ for which the minimal length $l_{\mathrm{p}}\left(\Gamma_{n}, \mathrm{~m}\right):=\min _{\gamma \in \Gamma_{n}} \sum_{v \in \gamma} \mathrm{m}(v) \geq 1$. Such $\mathrm{m}$, called admissible weights, form a convex set. The reciprocal of $\Lambda_{n}(\mathrm{p})$, called the $\mathrm{p}$-modulus of $\Gamma_{n}$, is found by minimization over this set of the ( $\mathrm{p}$-th power of) p-norm $\|\mathrm{m}\|_{\mathrm{p}}^{\mathrm{p}}:=\sum_{v \in \Gamma_{n}} \mathrm{~m}(v)^{\mathrm{p}} ;$ specifically:

$$
\Lambda_{n}(\mathrm{p})^{-1}=\inf \left\{\|\mathrm{m}\|_{\mathrm{p}}^{\mathrm{p}}: \mathrm{m}: \mathcal{V}_{n} \rightarrow[0, \infty) \text { admissible }\right\} .
$$

However, this convex minimization is inherently hampered by the huge number of admissibility constraints: one for each $\gamma \in \Gamma_{n}$. In contrast, the convex minimization for $R_{n}(\mathrm{p})$ only has local divergence constraints at the vertices in $\mathcal{V}_{n} \backslash\left(\mathcal{A}_{n} \cup \mathcal{B}_{n}\right)$ and a single total flux constraint. The cardinality of $\Gamma_{n}$ vastly exceeds that of $\mathcal{V}_{n}$ with increasing $n$.

In [26], Malo computed numerical approximations to $\Lambda_{n}(\mathrm{p})$ for $n=2,3,4,5$ and several values of $\mathrm{p}$ (including 1.7 and 1.8). The long running time for the algorithm limited the precision of $\Lambda_{n}(\mathrm{p})$ to two digits for $n=4$ and one significant digit for $n=$ 5. As a result the $10^{-1}$ wide bracketing of $\mathrm{p}_{\mathrm{el}}, 1.7 \leq \mathrm{p}_{\mathrm{el}} \leq 1.8$, had to be done based on the monotonicity properties of the numerical approximations to $\Lambda_{2}(\mathrm{p}), \Lambda_{3}(\mathrm{p}), \Lambda_{4}(\mathrm{p})$, $\Lambda_{5}(\mathrm{p})$, which were taken as an indirect indication of whether $\lim \sup _{n \rightarrow \infty} \Lambda_{n}(\mathrm{p})$ is finite or not. We were able to compute $R_{n}(\mathrm{p})$ to 8 digit precision for $n=1, \ldots, 8$ at the expense of less than 24 hours of a single core processing time (for each value of p). This represents roughly $10^{3}$ gain in speed and $10^{6}$ gain in precision. We report on those computations in Section 8 but outline their theoretical foundations below.

1.7. p-Ohm law and convex duality. Computation of the p-resistance $R_{n}(\mathrm{p})$ with explicit error bounds is readily done by adapting the Lagrange multipliers approach of the standard DC circuit theory. One introduces an auxiliary function $\mathcal{U}: \mathcal{V}_{n} \rightarrow \mathbf{R}$, called a potential, such that $\mathcal{U}$ is constant on $\mathcal{A}_{n}$ and $\mathcal{B}_{n}$, say $\left.\mathcal{U}\right|_{\mathcal{A}_{n}}=0$ and $\left.\mathcal{U}\right|_{\mathcal{B}_{n}}=U_{0}$ for some (yet to be determined) $U_{0} \in \mathbf{R}$. For every vertex $v \in \mathcal{V}_{n} \backslash\left(\mathcal{A}_{n} \cup \mathcal{B}_{n}\right), \mathcal{U}(v)$ plays a role of the multiplier enforcing $\operatorname{div} \mathcal{J}(v)=0$. The potential drop $U_{0}$ is the Lagrange multiplier for the flux constraint $J(\mathcal{J})=J_{0}$. This yields a Lagrangian (Section 2),

$$
\mathcal{L}(\mathcal{J}, \mathcal{U}):=U_{0} J_{0}+\sum_{e \in \mathcal{E}_{n}} \frac{r(e)^{\mathrm{p}^{*} / \mathrm{p}}}{\mathrm{p}^{*}}|\mathcal{J}(e)|^{\mathrm{p}^{*}}-\nabla \mathcal{U}(e) \mathcal{J}(e)
$$

where $\nabla \mathcal{U}$ is the discrete gradient of $\mathcal{U}$ (i.e. $\nabla \mathcal{U}(e):=\mathcal{U}\left(e_{+}\right)-\mathcal{U}\left(e_{-}\right)$with $e_{-}$and $e_{+}$denoting the initial and terminal vertices of $e$ ). The associated Euler-Lagrange equation is what we call $\mathrm{p}-\mathrm{Ohm}$ law:

$$
\nabla \mathcal{U}(e)=(r(e) \mathcal{J}(e))^{\mathrm{p}^{*} / \mathrm{p}} .
$$

Above, and elsewhere, $(t)^{\mathrm{p}^{*} / \mathrm{p}}$ is a shorthand for $\operatorname{sign}(t)|t|^{\mathrm{p}^{*} / \mathrm{p}}$. One seeks $\mathcal{J}: \mathcal{E}_{n} \rightarrow \mathbf{R}$ that is a flow from $\mathcal{A}_{n}$ to $\mathcal{B}_{n}$ and a potential $\mathcal{U}: \mathcal{V}_{n} \rightarrow \mathbf{R}$, including unknown $U_{0} \in$ 
$\mathbf{R}$, that solve the system consisting of (1.10) coupled with the following boundary conditions:

$$
J(\mathcal{J})=J_{0}=1,\left.\quad \mathcal{U}\right|_{\mathcal{A}_{n}}=0,\left.\quad \mathcal{U}\right|_{\mathcal{B}_{n}}=U_{0} .
$$

For $n \leq 8$, the number of unknowns reaches $10^{7}$ yet no more than 11 steps of a quasi-Newton method suffice to compute $R_{n}(\mathrm{p})$ with 8 digit precision.

In convex optimization nomenclature, simultaneously solving for the primal variable, $\mathcal{J}$, and its dual variable, $\mathcal{U}$, is called primal-dual approach (to the current power minimization). Explicit error bounds come from convex duality (which also enters other arguments). At the true optimum, we have

$$
R_{n}(\mathrm{p})^{1 / \mathrm{p}}=\frac{U_{0}}{P\left(\mathcal{U}_{\mathrm{opt}}\right)^{1 / \mathrm{p}}}=\frac{P\left(\mathcal{J}_{\mathrm{opt}}\right)^{1 / \mathrm{p}^{*}}}{J_{0}} \quad \text { with } \quad P\left(\mathcal{U}_{\mathrm{opt}}\right)=P\left(\mathcal{J}_{\mathrm{opt}}\right)=U_{0} J_{0}
$$

where (overloading the notation) $P(\mathcal{U})$ denotes the potential p-power defined as

$$
P(\mathcal{U}):=\sum_{e \in \mathcal{E}_{n}} \frac{|\nabla \mathcal{U}(e)|^{\mathrm{p}}}{r(e)} .
$$

For approximate optimizers $\mathcal{J}_{\text {approx }}$ and $\mathcal{U}_{\text {approx }}$ (really, any flow/potential pair satisfying the boundary conditions $)$, generally $P\left(\mathcal{U}_{\text {approx }}\right) \neq P\left(\mathcal{J}_{\text {approx }}\right)$, and the above duality relation relaxes to

$$
\frac{U_{0}}{P\left(\mathcal{U}_{\text {approx }}\right)^{1 / \mathrm{p}}} \leq R_{n}(\mathrm{p})^{1 / \mathrm{p}} \leq \frac{P\left(\mathcal{J}_{\text {approx }}\right)^{1 / \mathrm{p}^{*}}}{J_{0}} .
$$

The gap between the two extremes of (1.14) gives an explicit error bound on the approximation to $R_{n}(\mathrm{p})^{1 / \mathrm{p}}$.

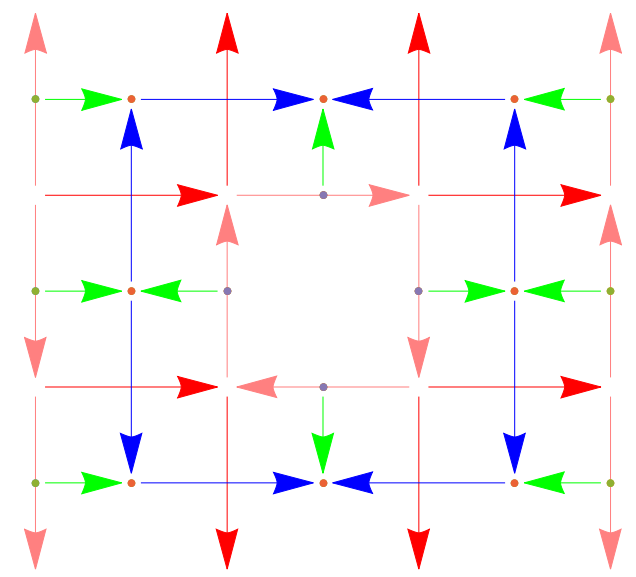

Figure 1.5. Network $\mathcal{G}_{1}$ (red and pink) and its topological dual $\mathcal{G}_{1}^{*}$ (blue and green). Edge resistance is $2^{-1}$ for red, $2^{\mathrm{p}^{*}} / \mathrm{p}$ for blue, and 1 for green and pink. (Midpoints of boundary edges of $\mathcal{G}_{1}$ are vertices of $\mathcal{G}_{1}^{*}$. Edge orientations are immaterial.)

1.8. Topological duality. The last conceptual piece underlying our techniques (and required to state the conjecture about the extremal metric) is the discrete analogue of the classical duality between the potential and the stream function in two-dimensional fluid dynamics. We start with dualizing the graphs $\mathcal{G}_{n}=\left(\mathcal{V}_{n}, \mathcal{G}_{n}\right)$ to obtain graphs $\mathcal{G}_{n}^{*}=\left(\mathcal{V}_{n}^{*}, \mathcal{E}_{n}^{*}\right)$. The edges in $\mathcal{E}_{n}^{*}$ and $\mathcal{E}_{n}$ are in bijective correspondence, and the edge dual to $e$ is denoted by $e^{*}$. In our renderings, see Figure 1.5, $e^{*}$ is obtained by rotating $e$ by $90^{\circ}$ about its midpoint, with the additional provisopurely for figure making convenience - that $e^{*}$ is shortened to half-length by clipping 
at the midpoint if that point is on the boundary of $X_{n}$. (Section 2 discusses dualizing in more detail, and the construction of $\mathcal{G}_{n}^{*}$ is in Section 4.) The resistances of the edges in $\mathcal{E}_{n}^{*}$ are determined by the rule

$$
r(e)^{1 / \mathrm{p}} r^{*}\left(e^{*}\right)^{1 / \mathrm{p}^{*}}=1 .
$$

To avoid confusion with the convex duality (between $\mathcal{J}$ and $\mathcal{U}$ ) we use the term topological duality and employ $*$ superscript. Yet, be forewarned that $*$ in object ${ }^{*}$ plays two distinct roles: it either signifies operation of taking the dual of object (e.g., $\mathcal{G}_{n}^{*}$ and $e^{*}$ are duals of $\mathcal{G}_{n}$ and $e$ ) or just serves as a reminder that object ${ }^{*}$ lives on the other side of the duality (as in $\mathcal{V}_{n}^{*}$ and $r^{*}(\cdot)$ ). Which is the case should be clear from the context.

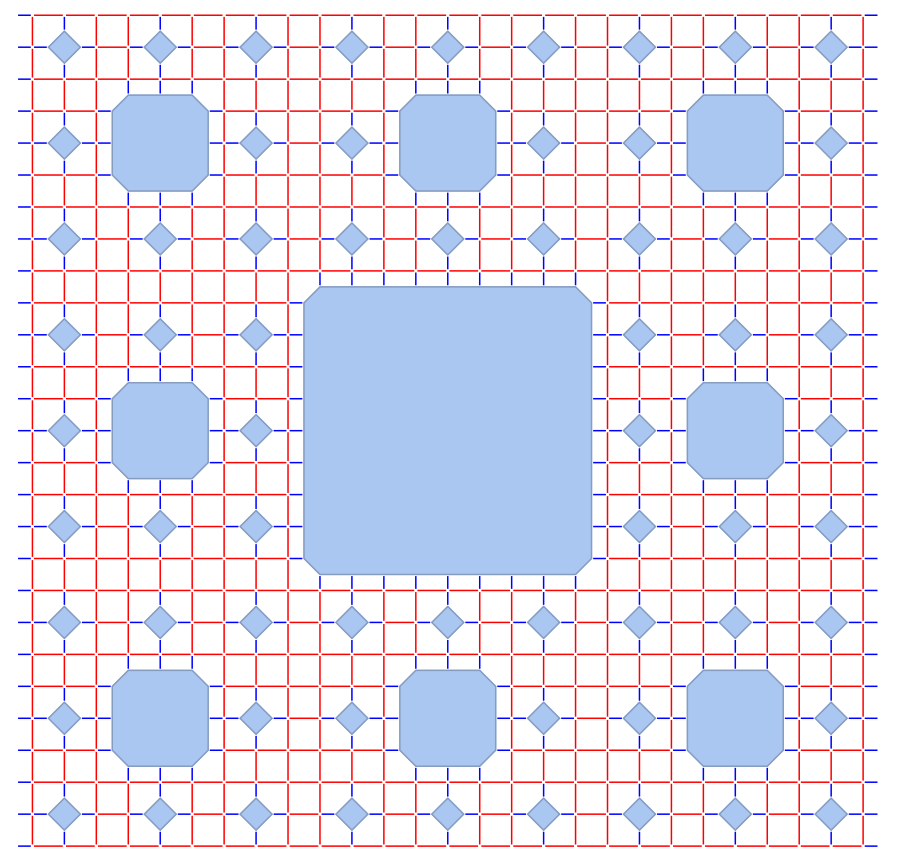

Figure 1.6. Network $\mathcal{G}_{3}^{*}$ with superconducting islands (blue). (Edge resistances are $r^{*}(e)=$ $2^{\mathrm{p}^{*} / \mathrm{p}}=2^{\mathrm{p}^{*}-1}$ for blue and $r^{*}(e)=1$ for red.)

Denoting by $\mathcal{A}_{n}^{*}$ and $\mathcal{B}_{n}^{*}$ the leftmost and rightmost vertices in $\mathcal{V}_{n}^{*}$ (respectively), we say that $\mathcal{J}^{*}$ is a flow from $\mathcal{A}_{n}^{*}$ to $\mathcal{B}_{n}^{*}$ iff $\operatorname{div} \mathcal{J}^{*}(v)=0$ for every $v \in \mathcal{V}_{n}^{*}$ that is not a on the boundary of $X_{n}$, with the additional proviso that, for any bounded component $C$ of $\mathbf{R}^{2} \backslash X_{n}$

$$
\operatorname{div} \mathcal{J}^{*}(C):=\sum_{v \in C} \operatorname{div} \mathcal{J}^{*}(v)=0
$$

Intuitively, $C$ shorts the terminals of the resistors in $\mathcal{E}_{n}^{*}$ touching $C$; we think of such $C$ as superconducting islands. The $\mathrm{p}^{*}$-power of a flow $\mathcal{J}^{*}$ is denoted by $P^{*}\left(\mathcal{J}^{*}\right)$, and reads (cf. (1.2))

$$
P^{*}\left(\mathcal{J}^{*}\right):=\sum_{e \in \mathcal{E}_{n}^{*}} r^{*}(e)^{\mathrm{p} / \mathrm{p}^{*}}\left|\mathcal{J}^{*}(e)\right|^{\mathrm{p}} .
$$

The $\mathrm{p}^{*}$-resistance from $\mathcal{A}_{n}^{*}$ to $\mathcal{B}_{n}^{*}$ results from minimizing over all $\mathcal{J}^{*}$ as above:

$$
R_{n}^{*}(\mathrm{p}):=\min _{\mathcal{J}^{*}} \frac{P^{*}\left(\mathcal{J}^{*}\right)^{\mathrm{p}^{*} / \mathrm{p}}}{J^{*}\left(\mathcal{J}^{*}\right)^{\mathrm{p}^{*}}},
$$


where $J^{*}\left(\mathcal{J}^{*}\right)$ is the flux of $\mathcal{J}^{*}$ from $\mathcal{A}_{n}^{*}$ to $\mathcal{B}_{n}^{*}$, which can again be normalized to $J^{*}\left(\mathcal{J}^{*}\right)=J_{0}^{*}=1$. (Note that we chose to treat $R_{n}^{*}(\mathrm{p})$ as a function of $\mathrm{p}$, not $\mathrm{p}^{*}$.) Just like we did for $\mathcal{G}_{n}, R_{n}^{*}(\mathrm{p})$ can be found via Lagrange multipliers, which yield p*-Ohm law

$$
\nabla \mathcal{U}^{*}\left(e^{*}\right)=\left(r^{*}\left(e^{*}\right) \mathcal{J}^{*}\left(e^{*}\right)\right)^{\mathrm{p} / \mathrm{p}^{*}} .
$$

Therefore, one seeks a flow $\mathcal{J}^{*}: \mathcal{E}_{n}^{*} \rightarrow \mathbf{R}$ from $\mathcal{A}_{n}^{*}$ to $\mathcal{B}_{n}^{*}$ and a potential $\mathcal{U}^{*}: \mathcal{V}_{n}^{*} \rightarrow \mathbf{R}$, including the potential drop $U_{0}^{*} \in \mathbf{R}$, solving the system consisting of (1.19) with boundary conditions

$$
J^{*}\left(\mathcal{J}^{*}\right)=J_{0}^{*}=1,\left.\quad \mathcal{U}^{*}\right|_{\mathcal{A}_{n}^{*}}=0,\left.\quad \mathcal{U}^{*}\right|_{\mathcal{B}_{n}^{*}}=U_{0}^{*},\left.\quad \mathcal{U}^{*}\right|_{C}=\operatorname{Const}(C)
$$

where $C$ ranges over all bounded components of the complement of $X_{n}$ (and Const $(C)$ is a $C$ dependent constant).

The upshot of the topological duality (Proposition 2.5) is the following relation between the resistances:

$$
R_{n}(\mathrm{p})^{1 / \mathrm{p}} R_{n}^{*}(\mathrm{p})^{1 / \mathrm{p}^{*}}=1
$$

What is more, the optimal currents and potentials are linked via

$$
\mathcal{J}^{*}\left(e^{*}\right)=\nabla \mathcal{U}(e) \text { and } \mathcal{J}(e)=\nabla \mathcal{U}^{*}\left(e^{*}\right) .
$$

1.9. Uniformization conjecture. We are ready to define the conjectural uniformizing map behind Figure 1.2. The idea is to use the potential $\mathcal{U}$ (associated to the top-to-bottom current $\mathcal{J}$ ) and its dual counterpart $\mathcal{U}^{*}$ as coordinates. One way to do this is to set

$$
F_{n}(v):=\left(\mathcal{U}(v), \mathcal{U}_{\text {avg }}^{*}(v)\right)
$$

where $\mathcal{U}_{\text {avg }}^{*}(v)$ is the average of $\mathcal{U}^{*}$ over the vertices of $\mathcal{G}_{n}^{*}$ that are near $v \in \mathcal{G}_{n}$. (In Figure 1.2, we used the vertices of $\mathcal{G}_{n}^{*}$ that belong to the $3^{-n} \times 3^{-n}$ square centered at $v$.)

Linear interpolation between the vertices extends $F_{n}$ to edges and yields a map defined on the underlying subset $\left|\mathcal{G}_{n}\right| \subset \mathbf{R}^{2}$ of the network $\mathcal{G}_{n}$, equipped with the Euclidean distance. $Y_{n}:=F_{n}\left(\left|\mathcal{G}_{n}\right|\right)$ is a connected subset of $\mathbf{R}^{2}$ (shown in Figure 1.2 for $n=5$ ) equipped with the path metric induced by the Euclidean distance.

Conjecture 1.5. (Uniformization) The maps $F_{n}:\left|\mathcal{G}_{n}\right| \rightarrow Y_{n}$ converge in the Gromov-Hausdorff sense to a quasi-symmetric homeomorphism $F: X \rightarrow X_{\text {slit }}$ where $X_{\text {slit }}$ is a planar slit Carpet (with the path metric induced by the Euclidean distance). The carpet is a quasi-symmetric uniformization of $X$ in the sense that $\operatorname{dim}_{H D}\left(X_{\text {slit }}\right)=$ $\operatorname{dim}_{A R}(X)=\mathrm{p}_{\text {res }}$. Moreover, the limits $R\left(\mathrm{p}_{\mathrm{res}}\right):=\lim _{n \rightarrow \infty} R_{n}\left(\mathrm{p}_{\mathrm{res}}\right)$ and $R^{*}\left(\mathrm{p}_{\mathrm{res}}\right):=$ $\lim _{n \rightarrow \infty} R_{n}^{*}\left(\mathrm{p}_{\mathrm{res}}\right)$ exist and the underlying rectangle of $X_{\text {slit }}$ has dimensions $R\left(\mathrm{p}_{\mathrm{res}}\right) \times$ $R^{*}\left(\mathrm{p}_{\text {res }}\right)$. (Note: from (1.21), if one of the limits exists so does the other.)

An analogous conjecture can be stated in a continuous PDE setting by using $F_{n}: X_{n} \rightarrow \mathbf{R}^{2}$ where $F_{n}(x, y):=\left(\mathcal{U}(x, y), \mathcal{U}^{*}(x, y)\right)$ is a pair of conjugate pres $^{-}$ harmonic functions on the approximant $X_{n}$ satisfying a continuous version of our boundary conditions. Whichever setting one chooses, the key to the existence of the optimal metric is likely to lie in a priori bounds on a suitable renormalization operator. A construction of such an operator will be given elsewhere. 


\section{Principles of DC circuits at exponent $p>1$}

2.1. Flows in resistor networks. A network of resistors, or simply a network, is a finite directed graph $\mathcal{G}$ with non-negative weights over the edges. It is formalized as a quintuple $\mathcal{G}=(\mathcal{V}, \mathcal{E},+,-, r)$. Here $\mathcal{V}$ and $\mathcal{E}$ are two finite sets, the vertex set and the edge set. $\pm: \mathcal{E} \mapsto \mathcal{V}$ are two maps, sending an edge $e$ to vertices denoted by $e_{-}$and $e_{+}$, respectively, and called the start and end vertices of $e$. $r: \mathcal{E} \rightarrow(0, \infty)$ is a function assigning to an edge $e$ weight $r(e)$, of which we think as the resistance of $e$. Incidentally, allowing $r(e)$ to also take values 0 and $\infty$ is often convenient but we do not do so at the outset to keep our formulations simpler. (In particular, this will simplify assertions about uniqueness of current/potential.) Although we prefer working with directed graphs, keep in mind that p-resistance will not depend on the orientation of the edges and we shall freely modify $\mathcal{G}$ by reversing some edges (which corresponds to an obvious modification of the quintuple). In particular, by a path we mean a path in the underlying undirected graph. To formalize this, call a sequence of edges $\gamma=\left(e_{1}, \ldots, e_{n}\right)$ a strong path in $\mathcal{G}$ if $e_{i}^{+}=e_{i+1}^{-}$for $i=1, \ldots, n-1$. Then a sequence of edges is a path if it can be turned into a strong path upon reversing some of the edges. There are two such strong paths, and picking one amounts to choosing an orientation of the path. Later we shall require that $\mathcal{G}$ is planar (for the sake of topological duality) but we do not make this assumption just yet.

We shall call functions $\mathcal{J}: \mathcal{E} \rightarrow \mathbf{R}$ vectorfields on $\mathcal{G}$ and define the divergence of $\mathcal{J}$ at $v \in \mathcal{V}$ as

$$
\operatorname{div} \mathcal{J}(v):=\sum_{e: e_{+}=v} \mathcal{J}(e)-\sum_{e: e_{-}=v} \mathcal{J}(e)
$$

We adopt the metaphor whereby $\mathcal{J}(e)$ is viewed as a flow rate of charges through $e$ (with $\mathcal{J}(e)>0$ when flowing from $e_{-}$to $e_{+}$). Thus $\operatorname{div} \mathcal{J}(v)$ is the excess of the inflow over the outflow (at the vertex $v$ ). Note that the net excess is zero, $\sum_{v \in \mathcal{V}} \operatorname{div} \mathcal{J}(v)=0$. (Sums over the empty set are defaulted to zero.) Also, reversing the orientation of $e$ and replacing $\mathcal{J}(e)$ by $-\mathcal{J}(e)$ will be immaterial.

Given a subset $\mathcal{O} \subset \mathcal{V}$, a vectorfield $\mathcal{J}: \mathcal{E} \rightarrow \mathbf{R}$ is a flow over $\mathcal{O}$ iff, for all $v \in \mathcal{O}, \operatorname{div} \mathcal{J}(v)=0$. This is to say that Kirchhoff's first law is satisfied at all $v \in \mathcal{O}$, the charge is conserved. In our considerations the network $\mathcal{G}$ will have a pair of distinguished disjoint non-empty subsets of vertices, $\mathcal{A}, \mathcal{B} \subset \mathcal{V}$, to which we refer as input and output sets, respectively. A flow in $\mathcal{G}$ from $\mathcal{A}$ to $\mathcal{B}$ is a flow over $\mathcal{O}:=\mathcal{V} \backslash(\mathcal{A} \cup \mathcal{B})$, and the associated flux (from $\mathcal{A}$ to $\mathcal{B}$ ) is

$$
J(\mathcal{J}):=-\sum_{v \in \mathcal{A}} \operatorname{div} \mathcal{J}(v)=\sum_{v \in \mathcal{B}} \operatorname{div} \mathcal{J}(v) .
$$

Note that the equality is a consequence of vanishing of divergence on $\mathcal{V} \backslash(\mathcal{A} \cup \mathcal{B})$ and that $J(\mathcal{J})>0$ intuitively means that the net flow is from $\mathcal{A}$ to $\mathcal{B}$.

We call $\mathcal{J}$ a unit flow from $\mathcal{A}$ to $\mathcal{B}$ iff $J(\mathcal{J})=1$. To ensure that a flow with nonzero flux exists we make a standing assumption that some vertex in $\mathcal{A}$ is connected to some vertex of $\mathcal{B}$, i.e., there is a path between the two. A triple $(\mathcal{G}, \mathcal{A}, \mathcal{B})$ is what we call a marked network. When there is no risk of confusion, the marking will be tacitly assumed and a flow in $\mathcal{G}$ from $\mathcal{A}$ to $\mathcal{B}$ will be simply referred to as a flow.

2.2. p-Resistance and power minimization. From now on, unless stated otherwise, $\mathrm{p}, \mathrm{p}^{*} \in(1, \infty)$ with $1 / \mathrm{p}+1 / \mathrm{p}^{*}=1$. Keep in mind that $\mathrm{p} / \mathrm{p}^{*}=\mathrm{p}-1$ and $\mathrm{p}^{*} / \mathrm{p}=\mathrm{p}^{*}-1$. The $\mathrm{p}$-power of $\mathcal{J}: \mathcal{E} \rightarrow \mathbf{R}$ is the $P(\mathcal{J})$ defined by $(1.2)$. 
Proposition 2.1. (Flow optimization and $\mathrm{p}$-Ohm law) For $\mathrm{p}>1$, given any $J_{0} \neq 0$, the following minimum over all flows from $\mathcal{A}$ to $\mathcal{B}$ with flux $J(\mathcal{J})=J_{0}$

$$
R(\mathrm{p}):=\min _{\mathcal{J}} \frac{P(\mathcal{J})^{\mathrm{p} / \mathrm{p}^{*}}}{J_{0}^{\mathrm{p}}}
$$

is attained at a unique flow $\mathcal{J}: \mathcal{E} \rightarrow \mathbf{R}$. This optimal flow $\mathcal{J}$ is uniquely characterized by the fact that it satisfies the $\mathrm{p}-O h m$ law (1.10) for some $\mathcal{U}: \mathcal{V} \rightarrow \mathbf{R}$ and $U_{0} \in \mathbf{R}$, subject to boundary conditions (1.11). Moreover, $R(\mathrm{p})$ does not depend on $J_{0}$ and is called the p-resistance of a marked network $(\mathcal{G}, \mathcal{A}, \mathcal{B})$.

Proof. Clearly, we may well minimize $P(\mathcal{J}) / J_{0}^{\mathrm{p}^{*}}$. The claimed independence on $J_{0}$ follows from invariance of this quotient under scaling of $\mathcal{J}$. The existence and uniqueness of the minimizing $\mathcal{J}$ is the result of $P(\cdot)$ being a strictly convex coercive function on the finite dimensional space of all $\mathcal{J}: \mathcal{E} \rightarrow \mathbf{R}$, in which the flows from $\mathcal{A}$ to $\mathcal{B}$ with the prescribed flux $J_{0}$ constitute a non-empty affine subspace. (Strict convexity follows from that of $t \mapsto|t|^{\mathrm{p}^{*}}$.)

If we introduce an auxiliary function $\mathcal{U}: \mathcal{V} \rightarrow \mathbf{R}$ as stipulated by the proposition (i.e. $\left.\mathcal{U}\right|_{\mathcal{A}}=0$ and $\left.\mathcal{U}\right|_{\mathcal{B}}=U_{0}$ for some variable $U_{0} \in \mathbf{R}$ ), then the Lagrangian accounting for the zero divergence and the flux constraints is

$$
\begin{aligned}
\mathcal{L}(\mathcal{J}, \mathcal{U}) & =\sum_{e \in \mathcal{E}} \frac{r(e)^{\mathrm{p}^{*} / \mathrm{p}}}{\mathrm{p}^{*}}|\mathcal{J}(e)|^{\mathrm{p}^{*}}-\sum_{v \notin \mathcal{A} \cup \mathcal{B}} \mathcal{U}(v) \operatorname{div} \mathcal{J}(v)-U_{0}\left(J(\mathcal{J})-J_{0}\right) \\
& =U_{0} J_{0}+\sum_{e \in \mathcal{E}} \frac{r(e)^{\mathrm{p}^{*} / \mathrm{p}}}{\mathrm{p}^{*}}|\mathcal{J}(e)|^{\mathrm{p}^{*}}-\nabla \mathcal{U}(e) \mathcal{J}(e)
\end{aligned}
$$

where $\nabla \mathcal{U}(e):=\mathcal{U}\left(e^{+}\right)-\mathcal{U}\left(e^{-}\right)$, and the second equality follows via summation by parts:

$$
\begin{aligned}
\sum_{e \in \mathcal{E}} \nabla \mathcal{U}(e) \mathcal{J}(e) & =\sum_{e \in \mathcal{E}}\left(\mathcal{U}\left(e_{+}\right)-\mathcal{U}\left(e_{-}\right)\right) \mathcal{J}(e) \\
& =\sum_{v \in \mathcal{V}} \mathcal{U}(v)\left(\sum_{e: e^{+}=v} \mathcal{J}(e)-\sum_{e: e^{-}=v} \mathcal{J}(e)\right) \\
& =\sum_{v \in \mathcal{V}} \mathcal{U}(v) \operatorname{div} \mathcal{J}(v) \\
& =\sum_{v \notin \mathcal{A} \cup \mathcal{B}} \mathcal{U}(v) \operatorname{div} \mathcal{J}(v)+\sum_{v \in \mathcal{B}} U_{0} \operatorname{div} \mathcal{J}(v) \\
& =\sum_{v \notin \mathcal{A} \cup \mathcal{B}} \mathcal{U}(v) \operatorname{div} \mathcal{J}(v)+U_{0} J(\mathcal{J}) .
\end{aligned}
$$

The p-Ohm law (1.10) is the Euler-Lagrange equation $\partial \mathcal{L} / \partial \mathcal{J}=0$. (The other Euler-Lagrange equation $\partial \mathcal{L} / \partial \mathcal{U}=0$ amounts to the divergence and flux constraints on $\mathcal{J}$.)

Finally, if a flow $\mathcal{J}$ and a function $\mathcal{U}$ satisfy the boundary conditions (for some $\left.J_{0}, U_{0}\right)$ as well as the p-Ohm law, then the Euler-Lagrange equations are satisfied. This makes $\mathcal{J}$ a relative critical point of the power function. By strict convexity, such a point is unique and hence coincides with the optimal flow.

We call the unique optimal flow in the proposition a p-current with flux $J_{0}$. 
Remark 2.2. From the proof, any flow $\mathcal{J}$ with $J(\mathcal{J})=0$ for which there is a potential $\mathcal{U}$ (satisfying the boundary conditions for some $U_{0}$ ) such that the p-Ohm law holds is a minimizer and thus must be constant and equal to zero, $\mathcal{J} \equiv 0$, by the strict convexity argument.

2.3. Flow-potential (convex) duality. As mentioned in the introduction, the p-resistance also arises from minimizing the power of functions $\mathcal{U}: \mathcal{V} \rightarrow \mathbf{R}$, called potentials. We shall consider two such functions $\mathcal{U}$ and $\mathcal{U}^{\prime}$ as equivalent iff $\mathcal{U}-\mathcal{U}^{\prime}$ is locally constant, i.e., $\nabla\left(\mathcal{U}-\mathcal{U}^{\prime}\right)(e)=0$ for all $e \in \mathcal{E}$. Thus the equivalence class of $\mathcal{U}$ is encoded by its gradient $\nabla \mathcal{U}: \mathcal{E} \rightarrow \mathbf{R}$. Such gradients are characterized among all functions $\mathcal{K}: \mathcal{E} \rightarrow \mathbf{R}$ by Kirchhoff's second law stipulating vanishing of the work along loops:

$$
\operatorname{work}_{\gamma}(\mathcal{K})=0 \quad(\forall \gamma \text { closed loop in } \mathcal{G}) .
$$

Here a loop is an oriented path that starts and ends at the same vertex. The work along an oriented path is $\operatorname{work}_{\gamma}(\mathcal{K}):=\sum_{i} \mathcal{K}\left(e_{i}\right)$ where $\left(e_{1}, \ldots, e_{n}\right)$ is the corresponding strong path (along which we replace $\mathcal{K}$ by $-\mathcal{K}$ for each reversed edge).

Proposition 2.3. (Potential optimization and convex duality) Given any $U_{0} \neq$ 0 , taking the p-power $P(\mathcal{U})$ defined as in (1.13), the p-resistance $R(\mathrm{p})$ defined by (2.1) coincides with the maximum of $\frac{U_{0}^{\mathrm{p}}}{P(\mathcal{U})}$ taken over over all $\mathcal{U}: \mathcal{V} \rightarrow \mathbf{R}$ satisfying $\left.\mathcal{U}\right|_{\mathcal{A}}=0$ and $\left.\mathcal{U}\right|_{\mathcal{B}}=U_{0}$

$$
R(\mathrm{p})=\max _{\mathcal{U}} \frac{U_{0}^{\mathrm{p}}}{P(\mathcal{U})}
$$

The maximum is attained at $\mathcal{U}$ that is unique (up to a locally constant function) and satisfies the p-Ohm law (1.10) for some flow $\mathcal{J}$ and $J_{0} \in \mathbf{R}$, subject to boundary conditions (1.11). Moreover, if $U_{0}, J_{0} \neq 0$, for any function $\mathcal{U}: \mathcal{V} \rightarrow \mathbf{R}$ and a flow $\mathcal{J}: \mathcal{E} \rightarrow \mathbf{R}$ satisfying boundary conditions (1.11), we have the convex duality gap bounds:

$$
\frac{U_{0}}{P(\mathcal{U})^{1 / \mathrm{p}}} \leq R(\mathrm{p})^{1 / \mathrm{p}} \leq \frac{P(\mathcal{J})^{1 / \mathrm{p}^{*}}}{J_{0}}
$$

Proof. As before, the function $\mathcal{K} \rightarrow \sum_{e \in \mathcal{E}} \frac{|\mathcal{K}(e)|^{\mathrm{p}}}{r(e)}$ is strictly convex and coercive on the space of all $\mathcal{K}: \mathcal{E} \rightarrow \mathbf{R}$. This space has a non-empty affine subspace consisting of all $\mathcal{K}$ that are gradients of $\mathcal{U}$ obeying the boundary conditions and that satisfy Kirchhoff's second law. Therefore $P(\mathcal{U})$ is minimized by some $\mathcal{U}$ that is unique up to the equivalence.

We have to produce a flow $\mathcal{J}$ satisfying the $\mathrm{p}-\mathrm{Ohm}$ law. The first order condition on the optimal $\mathcal{U}$ is $\left.\frac{d}{d \tau}\right|_{\tau=0} P(\mathcal{U}+\tau \delta \mathcal{U})=0$, with the arbitrary perturbation $\delta \mathcal{U}: \mathcal{V} \rightarrow \mathbf{R}$ satisfying $\left.\delta \mathcal{U}\right|_{\mathcal{A}}=\left.\delta \mathcal{U}\right|_{\mathcal{B}}=0$. It yields

$$
\sum_{e \in \mathcal{E}} \mathrm{p} \frac{(\nabla \mathcal{U}(e))^{\mathrm{p}-1}}{r(e)} \nabla \delta \mathcal{U}(e)=\mathrm{p} \sum_{v \in \mathcal{V}} \operatorname{div}\left(\frac{(\nabla \mathcal{U})^{\mathrm{p}-1}}{r}\right)(v) \delta \mathcal{U}(v)=0
$$

where we did summation by parts as in (2.2). (Recall that $(t)^{\mathrm{p}-1} \operatorname{stands}$ for $\operatorname{sign}(t)|t|^{\mathrm{p}-1}$ $=\frac{d}{d t}|t|^{\mathrm{p}}$.) As a result, any optimal $\mathcal{U}$ satisfies over $\mathcal{V} \backslash(\mathcal{A} \cup \mathcal{B})$ the discrete $\mathrm{p}$-Laplace equation:

$$
\operatorname{div}\left(\frac{(\nabla \mathcal{U})^{\mathrm{p}-1}}{r}\right)=0
$$


Such $\mathcal{U}$ are called p-harmonic on $\mathcal{V} \backslash(\mathcal{A} \cup \mathcal{B})$. Now, use the p-Ohm law to define a vectorfield $\mathcal{J}: \mathcal{E} \rightarrow \mathbf{R}$ associated to $\mathcal{U}, \mathcal{J}(e):=\frac{(\nabla \mathcal{U}(e))^{\mathrm{p} / \mathrm{p}^{*}}}{r(e)}$. This $\mathcal{J}$ is a flow due to (2.6). $\mathcal{J}$ is clearly non-zero if $U_{0} \neq 0$ and there is a path from $\mathcal{A}$ to $\mathcal{B}$, as we assumed. Then also $J_{0} \neq 0$ by Remark 2.2 .

To show the duality gap, for any $\mathcal{U}: \mathcal{V} \rightarrow \mathbf{R}$ and a flow $\mathcal{J}: \mathcal{E} \rightarrow \mathbf{R}$ (from $\mathcal{A}$ to $\mathcal{B})$ satisfying boundary conditions $(1.11)$ with the $U_{0} \neq 0$ and $J_{0} \neq 0$, equality $(2.2)$ and Hölder inequality yield

$$
U_{0} J_{0}=\sum_{e} \nabla \mathcal{U}(e) \mathcal{J}(e) \leq P(\mathcal{J})^{1 / \mathrm{p}^{*}} P(\mathcal{U})^{1 / \mathrm{p}}
$$

This gives the bounds (2.5). In particular, $\max _{\mathcal{U}} \frac{U_{0}}{P(\mathcal{U})^{1 / \mathrm{p}}} \leq R(\mathrm{p})^{1 / \mathrm{p}} \leq \min _{\mathcal{J}} \frac{P(\mathcal{J})^{1 / \mathrm{p}^{*}}}{J_{0}}$. To get (2.4), note that these are in fact equalities because Hölder inequality becomes an equality if we let $\mathcal{U}$ be optimal and $\mathcal{J}$ be the associated flow. (Also, by further scaling of $\mathcal{J}$, we can adjust its flux to any desired $J_{0} \neq 0$.)

When $\mathcal{J}$ and $\mathcal{U}$ are related by the p-Ohm equation, we call $\mathcal{U}$ a potential of $\mathcal{J}$ and say that $\mathcal{J}$ is induced by $\mathcal{U}$. If $\mathcal{J}$ is additionally a flow from $\mathcal{A}$ to $\mathcal{B}$ and $\mathcal{U}$ satsfies $\left.\mathcal{U}\right|_{\mathcal{A}}=0$ and $\left.\mathcal{U}\right|_{\mathcal{B}}=U_{0}$ (for some $U_{0} \neq 0$ ) then we call $(\mathcal{J}, \mathcal{U})$ a current-potential pair for the marked network $(\mathcal{G}, \mathcal{A}, \mathcal{B})$. Note that then $\mathcal{J}$ is a p-current (is optimal) and $\mathcal{U}$ is p-harmonic.

Corollary 2.4. (Joule's law) If $\mathcal{J}$ and $\mathcal{U}$ are a current-potential pair then $P(\mathcal{J})=P(\mathcal{U})$, and the common value $P:=P(\mathcal{J})=P(\mathcal{U})$ satisfies

$$
P=J_{0} U_{0}=\left(\frac{U_{0}}{R(\mathrm{p})^{1 / \mathrm{p}}}\right)^{\mathrm{p}}=\left(R(\mathrm{p})^{1 / \mathrm{p}} J_{0}\right)^{\mathrm{p}^{*}} .
$$

Proof. That $P(\mathcal{J})=P(\mathcal{U})$ follows from the $\mathrm{p}$-Ohm law and the definitions of the powers. Because we have equality in $(2.7), U_{0} J_{0}=P^{1 / \mathrm{p}} P^{1 / \mathrm{p}^{*}}=P$. The other equalities follow from the two characterizations of the resistance, $R(\mathrm{p})^{1 / \mathrm{p}}=U_{0} / P^{1 / \mathrm{p}}$ and $R(\mathrm{p})^{1 / \mathrm{p}}=P^{1 / \mathrm{p}^{*}} / J_{0}$.

2.4. Simple network modifications. When computing p-resistance some degree of simplification can be achieved by modifying a marked network so that the resistance is unchanged. We shall refer to such modified networks as equivalent. Below we list a few useful equivalences, with an indication of how to transform the currents and their potentials between the two networks. Sometimes the transformation can be already done at the level of arbitrary flows. Note that, for the resistance to be unaffected, it suffices that the boundary conditions and the powers (of the flow and the potential) are preserved under the transformation.

One trivial move is reversing a single edge $e$. Denoting the reversed edge by $e_{\text {new }}$, the correspondence of currents (even vectorfields) is given by $\mathcal{J}_{\text {new }}\left(e_{\text {new }}\right)=-\mathcal{J}(e)$ (with $\mathcal{J}_{\text {new }}=\mathcal{J}$ otherwise). The potential $\mathcal{U}$ is to be unchanged.

An even simpler move is removal of zero current edges, the edges where the current is a priori zero (e.g., by symmetry considerations). This is why including in $\mathcal{G}_{n}$ the horizontal edges along the top and bottom is immaterial for the top-to-bottom current (cf. Figure 1.3).

If there is a vertex $v$ that has only one incoming edge $e_{1}$ and one outgoing edge $e_{2}$ (so $e_{1}^{+}=v=e_{2}^{-}$) then one can remove $v$ and join $e_{1}$ and $e_{2}$, that is replace them by a single edge $e_{12}$ from $v_{1}:=e_{1}^{-}$to $v_{2}:=e_{2}^{+}$with the resistance given by the serial 
law:

$$
r\left(e_{12}\right)^{\mathrm{p}^{*} / p}=r\left(e_{1}\right)^{\mathrm{p}^{*} / \mathrm{p}}+r\left(e_{2}\right)^{\mathrm{p}^{*} / \mathrm{p}} .
$$

The power preserving current correspondence is then given by $\mathcal{J}\left(e_{12}\right)=\mathcal{J}\left(e_{1}\right)=$ $\mathcal{J}\left(e_{2}\right)$ (the latter equality dictated by Kirchhoff's first law). As to the potential $\mathcal{U}$, it remains unchanged after the joining, apart from forgetting the value at $v$. The opposite of joining amounts to dividing an edge $e$ into $e_{1}$ and $e_{2}$ meeting at a new vertex $v$. The current through $e_{i}$ is obvious, and the new potential value $\mathcal{U}(v)$ is uniquely determined by $\mathcal{U}\left(e^{-}\right)$and $\mathcal{U}\left(e^{+}\right)$and the condition $\nabla \mathcal{U}\left(e_{1}\right) / r\left(e_{1}\right)^{\mathrm{p}^{*}-1}=$ $\nabla \mathcal{U}\left(e_{2}\right) / r\left(e_{2}\right)^{\mathrm{p}^{*}-1}$, which is dictated by the equality of currents and the p-Ohm law.
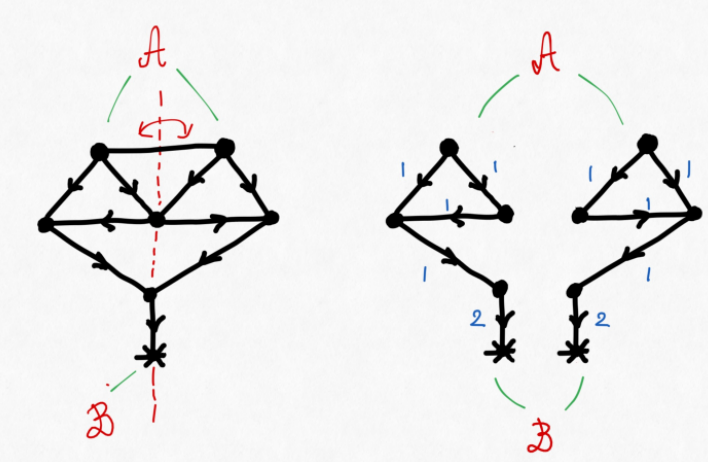

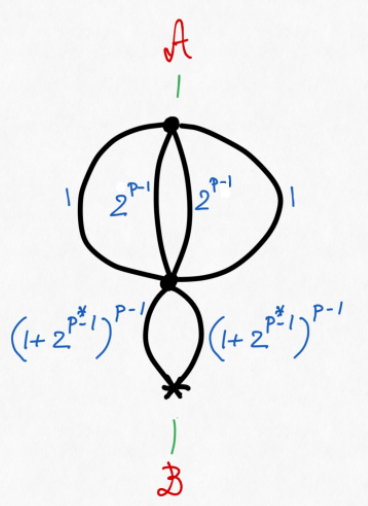

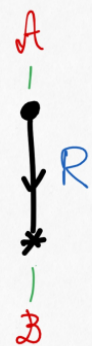

§

Figure 2.7. A network of unit resistors and its modifications by: removing a zero current edge at the top and splitting into symmetric halves, joining serial edges and merging symmetric vertices, merging parallel edges and one more serial joining. Edge resistances are in blue. The resistance from $\mathcal{A}$ to $\mathcal{B}$, computed via parallel and serial laws, is $R=\frac{1}{2}\left(\left(1+2^{1-\mathrm{p}}\right)^{1-\mathrm{p}^{*}}+\left(1+2^{\mathrm{p}^{*}-1}\right)\right)^{\mathrm{p}-1}$.

Presented with two parallel edges, i.e., $e_{1}$ and $e_{2}$ connecting the same vertices, $v_{1}:=e_{i}^{-}$and $v_{2}:=e_{i}^{+}(i=1,2), e_{1}$ and $e_{2}$ can be merged, that is replaced by a single edge $e_{12}$ (with $e_{12}^{-}=v_{1}$ and $e_{12}^{+}=v_{2}$ ) whose resistance is given by the parallel law:

$$
\frac{1}{r\left(e_{12}\right)}=\frac{1}{r\left(e_{1}\right)}+\frac{1}{r\left(e_{2}\right)}
$$

If we keep the potential unchanged, this law guarantees that its power is preserved. The power preserving current correspondence is dictated via the $\mathrm{p}$-Ohm law by the potential drop $U:=\mathcal{U}\left(v_{2}\right)-\mathcal{U}\left(v_{1}\right)$, specifically, $\mathcal{J}\left(e_{12}\right)^{\mathrm{p}^{*} / \mathrm{p}}=\frac{U}{r\left(e_{12}\right)}=\frac{U}{r\left(e_{1}\right)}+\frac{U}{r\left(e_{2}\right)}=$ $\left(\mathcal{J}\left(e_{1}\right)\right)^{\mathrm{p}^{*} / \mathrm{p}}+\left(\mathcal{J}\left(e_{2}\right)\right)^{\mathrm{p}^{*} / \mathrm{p}}$. Note that the correspondence of currents goes both ways: given $\mathcal{J}\left(e_{12}\right)$, there is a unique pair $\left(\mathcal{J}\left(e_{1}\right), \mathcal{J}\left(e_{2}\right)\right)$ that satisfies the above equation and $\mathcal{J}\left(e_{1}\right)+\mathcal{J}\left(e_{2}\right)=\mathcal{J}\left(e_{12}\right)$. (This used $\mathrm{p}>1$.) In particular, there is an obvious way to reverse merging: any edge can be also split into two parallel edges (with any resistances satisfying (2.10)).

In some circumstances, particularly in presence of symmetry, one can also merge and split vertices (while judiciously apportioning the edges to respect the Kirchhoff's first law). For instance, the first move in Figure 2.7 splits the three vertices on the axis of symmetry, and the second move merges three pairs of symmetric vertices. We shall not formalize this operation but only mention that we used it to confine numerical computations to the lower-left quarter of $\mathcal{G}_{n}$, thus lowering the memory requirement fourfold. 
Figure 2.7 gives an example of a reduction of a marked network to a single resistor by using a sequence of basic modifications. For more complex networks, only partial reduction can be achieved and one cannot escape numerical optimization to find the resistance.

2.5. Topological duality of planar circuits. Topological duality of networks should be thought of as the classical duality of planar graphs with an additional stipulation about resistances. We formalize it by defining what it means for two marked networks to be dual in a way that makes it easy to see the duality between $\mathcal{G}_{n}$ and $\mathcal{G}_{n}^{*}$ (the two networks we use to approximate the Sierpiński carpet). To make $\mathcal{G}_{n}^{*}$ less ad hoc, we outline the construction of the dual for a restricted class of planar networks that includes $\mathcal{G}_{n}$.

First, recall that a current-potential pair, with the potential up to equivalence (i.e. addition of a locally constant function), is encoded by a pair of vector-fields $(\mathcal{J}, \mathcal{K})$ where $\mathcal{J}$ and $\mathcal{K}=\nabla \mathcal{U}$ satisfy Kirchhoff's first and second laws, respectively, and are linked by the $\mathrm{p}$-Ohm law $(1.10),(\mathcal{K})^{\mathrm{p}}=(r \mathcal{J})^{\mathrm{p}^{*}}$. The boundary conditions (1.11) amount to additionally requiring that the flux of $\mathcal{J}$ is $J_{0}$ and the work of $\mathcal{K}$ along any path from any vertex of $\mathcal{A}$ to any vertex of $\mathcal{B}$ is equal to the fixed potential drop $U_{0}$. For brevity, let us refer to this second requirement as: the work from $\mathcal{A}$ to $\mathcal{B}$ is $U_{0}$.

Given two marked networks $(\mathcal{G}, \mathcal{A}, \mathcal{B})$ and $\left(\mathcal{G}^{*}, \mathcal{A}^{*}, \mathcal{B}^{*}\right)$, we call them topologically dual iff the following conditions hold. There is a bijection $\mathcal{E} \rightarrow \mathcal{E}^{*}$ between the edge sets such that the resistances of $e \in \mathcal{E}$ and its counterpart $e^{*} \in \mathcal{E}^{*}$ satisfy (1.15) and the induced correspondence between vectorfields on $\mathcal{G}$ and $\mathcal{G}^{*}$ exchanges Kirchhoff's laws and the boundary conditions in a way codified below.

For any $\mathcal{J}: \mathcal{E} \rightarrow \mathbf{R}$ and $\mathcal{K}^{*}: \mathcal{E}^{*} \rightarrow \mathbf{R}$ such that $\mathcal{J}(e)=\mathcal{K}^{*}\left(e^{*}\right)$ for all $e \in \mathcal{E}$, we require that: $\mathcal{J}$ satisfies Kirchhoff's first law on $\mathcal{V} \backslash(\mathcal{A} \cup \mathcal{B})$ (i.e. is a flow in $\mathcal{G}$ ) iff $\mathcal{K}^{*}$ satisfies Kirchhoff's second law on $\mathcal{G}^{*}$ (i.e. is a gradient of a potential on $\mathcal{G}^{*}$, cf. $(2.3)$ ). Likewise, we require that $\mathcal{K}^{*}$ satisfies Kirchhoff's first law on $\mathcal{V}^{*} \backslash\left(\mathcal{A}^{*} \cup \mathcal{B}^{*}\right)$ iff $\mathcal{J}$ satisfies Kirchhoff's second law on $\mathcal{G}$. Moreover, we demand that the flux of $\mathcal{J}$ from $\mathcal{A}$ to $\mathcal{B}$ equals the work of $\mathcal{K}^{*}$ from $\mathcal{A}^{*}$ to $\mathcal{B}^{*}$, and that the work of $\mathcal{J}$ from $\mathcal{A}$ to $\mathcal{B}$ equals the flux of $\mathcal{K}^{*}$ from $\mathcal{A}^{*}$ to $\mathcal{B}^{*}$.

Proposition 2.5. (Topological duality) Suppose that $(\mathcal{G}, \mathcal{A}, \mathcal{B})$ and $\left(\mathcal{G}^{*}, \mathcal{A}^{*}, \mathcal{B}^{*}\right)$ are topologically dual marked networks. Current-potential pairs $(\mathcal{J}, \mathcal{U})$ for $(\mathcal{G}, \mathcal{A}, \mathcal{B})$ and current-potential pairs $\left(\mathcal{J}^{*}, \mathcal{U}^{*}\right)$ for $\left(\mathcal{G}^{*}, \mathcal{A}^{*}, \mathcal{B}^{*}\right)$ are in bijective correspondence given by the following relations:

$$
\begin{array}{cll}
\mathcal{J}^{*}\left(e^{*}\right)=\nabla \mathcal{U}(e) & \text { and } & \mathcal{J}(e)=\nabla \mathcal{U}^{*}\left(e^{*}\right), \\
J_{0}^{*}=U_{0} & \text { and } & J_{0}=U_{0}^{*}
\end{array}
$$

Moreover, all four powers are equal: $P^{*}\left(\mathcal{J}^{*}\right)=P(\mathcal{U})=P(\mathcal{J})=P^{*}\left(\mathcal{U}^{*}\right)$, and the p-resistance from $\mathcal{A}$ to $\mathcal{B}$ and the $\mathrm{p}^{*}$-resistance from $\mathcal{A}^{*}$ to $\mathcal{B}^{*}$ satisfy

$$
R(\mathrm{p})^{1 / \mathrm{p}} R^{*}(\mathrm{p})^{1 / \mathrm{p}^{*}}=1
$$

Proof. Suppose that $(\mathcal{J}, \mathcal{U})$ is the current-potential pair for $(\mathcal{G}, \mathcal{A}, \mathcal{B})$ with drop $U_{0}$ and flux $J_{0}$. Then the vectorfield $\mathcal{K}^{*}: \mathcal{E}^{*} \rightarrow \mathbf{R}$ given by $\mathcal{K}^{*}\left(e^{*}\right):=\mathcal{J}(e)$ satsfies Kirchhoff's second law (because $\mathcal{J}$ satisfies the first law) and thus is of the form $\mathcal{K}^{*}=\nabla \mathcal{U}^{*}$ for some $\mathcal{U}^{*}: \mathcal{V}^{*} \rightarrow \mathbf{R}$ with $\left.\mathcal{U}^{*}\right|_{\mathcal{A}^{*}}=0$. Likewise, the vectorfield $\mathcal{J}^{*}: \mathcal{E}^{*} \rightarrow \mathbf{R}$ given by $\mathcal{J}^{*}\left(e^{*}\right):=\nabla \mathcal{U}(e)$ satisfies Kirchhoff's first law so is a flow. The formulas (2.11) are satisfied by construction and the p-Ohm law (1.10) translates 
to the $\mathrm{p}^{*}$-Ohm law $(1.19)$, making $\left(\mathcal{J}^{*}, \mathcal{U}^{*}\right)$ a current-potential pair. Moreover, by the flux/work stipulation in the definition of topological duality, $\mathcal{U}^{*}$ is constant equal to $J_{0}$ on $\mathcal{B}^{*},\left.\mathcal{U}^{*}\right|_{\mathcal{B}^{*}}=J_{0}$; so $U_{0}^{*}=J_{0}$. Using the stipulation again, the flux $J_{0}^{*}$ of $\mathcal{J}^{*}$ from $\mathcal{A}^{*}$ to $\mathcal{B}^{*}$ equals $U_{0}$; so $(2.12)$ holds.

To see the bijectivity, symmetric arguments can be made starting with a currentpotential pair $\left(\mathcal{J}^{*}, \mathcal{U}^{*}\right)$ for $\left(\mathcal{G}^{*}, \mathcal{A}^{*}, \mathcal{B}^{*}\right)$.

Finally, the equality of all four powers is immediate from their definitions and (1.15). (2.13) then follows from the definitions of the resistances in terms of currents and potentials (namely, (2.1) and (2.4) and their starred counterparts).

To shed light on how the dual network of a given marked network $(\mathcal{G}, \mathcal{A}, \mathcal{B})$ is constructed, let us describe the process under the assumption that $\mathcal{G}$ is connected and planar and each of $\mathcal{A}$ and $\mathcal{B}$ is a single vertex, or can be coalesced into a single vertex while preserving planarity of the network. Such are all the marked networks we use to approximate the carpet, including $\left(\mathcal{G}_{n}, \mathcal{A}_{n}, \mathcal{B}_{n}\right)$ (as in Figures 1.3 and 2.8).

Embed $\mathcal{G}$ into the closed unit disk $\overline{\mathbf{D}}:=\left\{(x, y): x^{2}+y^{2} \leq 1\right\}$ so that only the two input/output vertices, $\mathcal{A}$ and $\mathcal{B}$, sit on the boundary circle and the remaining vertices in $\mathcal{V}$ sit in the interior of $\overline{\mathbf{D}}$. To get the graph of $\mathcal{G}^{*}$, first place a single vertex in each connected component of what remains of $\overline{\mathbf{D}}$ after removing the edges. Further, place an edge $e^{*}$ between the vertices of any two components abutting along an edge $e \in \mathcal{E}$. The two input/output vertices $\mathcal{A}^{*}$ and $\mathcal{B}^{*}$ of thus constructed $\mathcal{G}^{*}$ are the vertices of the two components that contain arcs of the boundary circle. (The orientation of the circle determines which is which.)

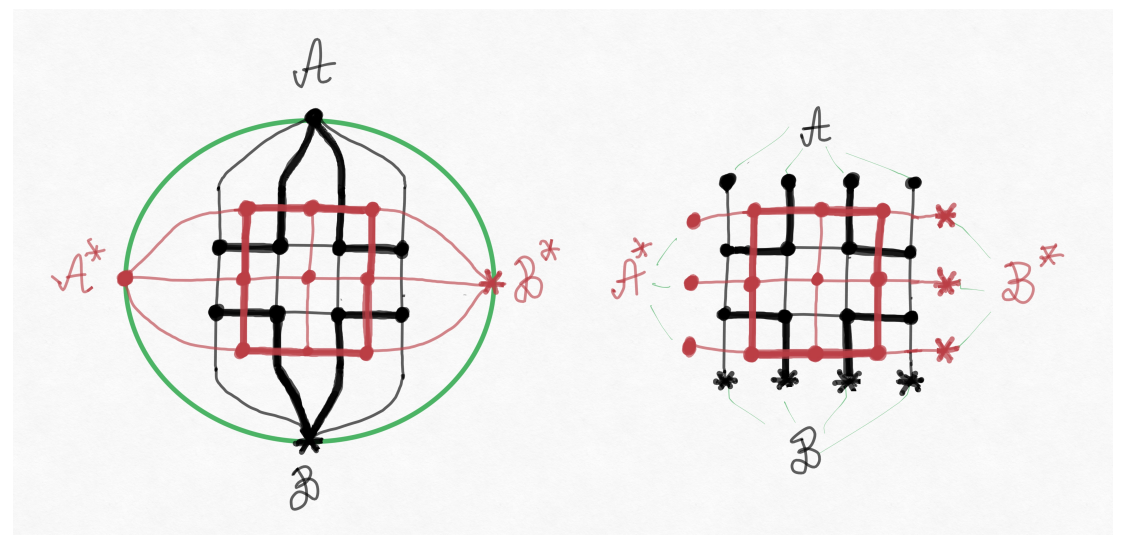

Figure 2.8. Two (equivalent) pairs, $(\mathcal{G}, \mathcal{A}, \mathcal{B})$ and $\left(\mathcal{G}^{*}, \mathcal{A}^{*}, \mathcal{B}^{*}\right)$, of topologically dual marked networks. (The one on the left is embedded in a closed disk bounded by the green circle.) The edge resistance is $2^{-1}$ for thick black and $2^{\mathrm{p}^{*} / \mathrm{p}}$ for thick red; all other resistances are unit.

Example. Figure 2.8 depicts two pairs of dual marked networks $(\mathcal{G}, \mathcal{A}, \mathcal{B})$ and $\left(\mathcal{G}^{*}, \mathcal{A}^{*}, \mathcal{B}^{*}\right)$. The left pair is obtained from the right one by merging of the vertices of the input/output sets. Comparing with Figure 1.5 , note that $(\mathcal{G}, \mathcal{A}, \mathcal{B})$ is also equivalent to the level $n=1$ standard approximation $\left(\mathcal{G}_{1}, \mathcal{A}_{1}, \mathcal{B}_{1}\right)$ to the carpet. Likewise, splitting the center vertex of the $\mathcal{G}^{*}$ on the right into four new vertices yields $\mathcal{G}_{1}^{*}$. The only caveat is that, to guarantee a natural correspondence between flows over $\mathcal{V}^{*} \backslash\left(\mathcal{A}^{*} \cup \mathcal{B}^{*}\right)$ and flows over $\mathcal{V}_{1}^{*} \backslash\left(\mathcal{A}_{1}^{*} \cup \mathcal{B}_{1}^{*}\right)$ we have to require that the latter have vanishing flux through the set of four vertices on the boundary of the central square, the superconducting island. With this additional stipulation the duality relations stated in Proposition 2.5 hold for $\left(\mathcal{G}_{1}, \mathcal{A}_{1}, \mathcal{B}_{1}\right)$ and $\left(\mathcal{G}_{1}^{*}, \mathcal{A}_{1}^{*}, \mathcal{B}_{1}^{*}\right)$. 
One can see that, for all $n \geq 1$, the marked networks $\left(\mathcal{G}_{n}, \mathcal{A}_{n}, \mathcal{B}_{n}\right)$ and $\left(\mathcal{G}_{n}^{*}, \mathcal{A}_{n}^{*}, \mathcal{B}_{n}^{*}\right)$, discussed in the introduction, are topologically dual provided one modifies $\mathcal{G}_{n}^{*}$ by coalescing the vertices on the boundary of every removed square into one vertex, or (equivalently) imposes the superconducting boundary conditions, as we did in (1.20). Formal proofs of duality of $\left(\mathcal{G}_{n}, \mathcal{A}_{n}, \mathcal{B}_{n}\right)$ and $\left(\mathcal{G}_{n}^{*}, \mathcal{A}_{n}^{*}, \mathcal{B}_{n}^{*}\right)$ are done by induction on $n \in \mathbf{N}$, which is straightforward by the recursive constructions of the networks via substitutions $\Phi_{\text {std }}$ and $\Phi$ defined in Sections 6 and 4, respectively. We leave the details as an exercise.

\section{Resistance/length estimate (Th. 1.2)}

As already mentioned, Theorem 1.2 depends on the equality $p_{\mathrm{el}}=\operatorname{dim}_{A R}(X)$, shown in [26]. In view of the definitions of $p_{e l}$ and $p_{\text {res }}$ (see (1.7) and (1.4)), it suffices to show that $R_{n}(\mathrm{p})$ and $\Lambda_{n}(\mathrm{p})$ are comparable up to a multiplicative constant independent of $n$ and $\mathrm{p}$. This is the content of the following result, which applies to any marked networks.

Theorem 3.1. Suppose that a sequence of marked networks $\left(\mathcal{G}_{n}, \mathcal{A}_{n}, \mathcal{B}_{n}\right)_{n \in \mathbf{N}}$ satisfies uniform bounds on resistances and maximum degrees: $0<r_{\min } \leq r(e) \leq$ $r_{\max }$ for all $e \in \mathcal{E}_{n}$ and $\max _{v \in \mathcal{V}_{n}} \#\left\{e \in \mathcal{E}_{n}: v \in \partial e\right\} \leq D$ for all $n \in \mathbf{N}$. (Here $\partial e:=\left\{e_{-}, e_{+}\right\}$are the ends of e.) Then, for $\mathrm{p}>1$,

$$
\frac{1}{2 D^{\mathrm{p}-1} r_{\max }} R_{n}(\mathrm{p}) \leq \Lambda_{n}(\mathrm{p}) \leq \frac{2^{\mathrm{p}-1} D^{\mathrm{p}^{2}-\mathrm{p}+1}}{r_{\min }} R_{n}(\mathrm{p})
$$

In particular, the critical exponents for the p-resistance of $\left(\mathcal{G}_{n}, \mathcal{A}_{n}, \mathcal{B}_{n}\right)$ and the $\mathrm{p}$-extremal length of the paths connecting $\mathcal{A}_{n}$ to $\mathcal{B}_{n}$ coincide, i.e., $\mathrm{p}_{\mathrm{el}}=\mathrm{p}_{\mathrm{res}}$.

To make a connection with [2], of which we became aware only while revising the manuscript, Th. 4.2 there implies that the p-resistance coincides with the edgeweighted version of the p-extremal length. Thus Theorem 3.1 can be viewed as an assertion about essential equivalence of the latter with the vertex-weighted version of the p-extremal length we use.

For the standard approximations to the carpet, we have $r_{\min }=1 / 2, r_{\max }=1$, and $D=4$. Other sequences of networks we use have similar uniform bounds.

The rest of this section is devoted to the proof of the theorem. Since $p_{e l}=p_{\text {res }}$ is immediate from inequalities (3.1), we only have to attend to the latter. Recall that the family of all vertex sets of paths connecting $\mathcal{A}_{n}$ to $\mathcal{B}_{n}$ was denoted by $\Gamma_{n}$. Its p-extremal length is (cf. (1.6) and (1.8))

$$
\Lambda_{n}(\mathrm{p})=\max _{\mathrm{m}} \frac{l_{\mathrm{p}}\left(\Gamma_{n}, \mathrm{~m}\right)^{\mathrm{p}}}{\|\mathrm{m}\|_{\mathrm{p}}^{\mathrm{p}}}
$$

where $l_{\mathrm{p}}\left(\Gamma_{n}, \mathrm{~m}\right):=\min _{\gamma \in \Gamma_{n}} \sum_{v \in \gamma} \mathrm{m}(v)$ is called the m-length of $\Gamma_{n}$.

Proof of the left inequality in (3.1). Consider any potential $\mathcal{U}: \mathcal{V}_{n} \rightarrow \mathbf{R}$ satisfying the boundary conditions $\left.\mathcal{U}\right|_{\mathcal{A}_{n}}=0$ and $\left.\mathcal{U}\right|_{\mathcal{B}_{n}}=U_{0}$ with $U_{0}>0$. Define a weight $\mathrm{m}: \mathcal{V}_{n} \rightarrow[0, \infty)$ by setting

$$
\mathrm{m}(v):=\sum_{e \in \mathcal{E}_{n}: v \in \partial e}|\nabla \mathcal{U}(e)| .
$$


First note that $l_{\mathrm{p}}\left(\Gamma_{n}, \mathrm{~m}\right) \geq U_{0}$ because, if $\gamma \in \Gamma_{n}$ is a path with vertices $\left(v_{0}, \ldots, v_{l}\right)$ and edges $\left(e_{1}, \ldots, e_{l}\right)$ (so that $\left.\partial e_{i}=\left\{v_{i-1}, v_{i}\right\}\right)$, then

$$
\sum_{v \in \gamma} \mathrm{m}(v) \geq \sum_{i=1}^{l}\left|\nabla \mathcal{U}\left(e_{i}\right)\right| \geq \mathcal{U}\left(v_{l}\right)-\mathcal{U}\left(v_{0}\right)=U_{0} .
$$

Second, by using the convexity of $t \mapsto t^{\mathrm{p}}$, we estimate

$$
\begin{aligned}
\sum_{v \in \mathcal{V}_{n}} \mathrm{~m}(v)^{\mathrm{p}} & =\sum_{v \in \mathcal{V}_{n}}\left(\sum_{e \in \mathcal{E}_{n}: v \in \partial e}|\nabla \mathcal{U}(e)|\right)^{\mathrm{p}} \leq \sum_{v \in \mathcal{V}_{n}} D^{\mathrm{p}-1}\left(\sum_{e \in \mathcal{E}_{n}: v \in \partial e}|\nabla \mathcal{U}(e)|^{\mathrm{p}}\right) \\
& =2 D^{\mathrm{p}-1} \sum_{e \in \mathcal{E}_{n}}|\nabla \mathcal{U}(e)|^{\mathrm{p}} \leq 2 D^{\mathrm{p}-1} r_{\max } P(\mathcal{U})
\end{aligned}
$$

where the factor 2 is due to every edge having two ends. Inequalities (3.4) and (3.5) yield

$$
\Lambda_{n}(\mathrm{p})=\max _{\mathrm{m}} \frac{l_{\mathrm{p}}\left(\Gamma_{n}, \mathrm{~m}\right)^{\mathrm{p}}}{\|\mathrm{m}\|_{\mathrm{p}}^{\mathrm{p}}} \geq\left(2 D^{\mathrm{p}-1} r_{\max }\right)^{-1} \frac{U_{0}^{\mathrm{p}}}{P(\mathcal{U})} .
$$

Via the potential based characterization of $R_{n}(\mathrm{p})$ given by (2.4), maximizing over $\mathcal{U}$ yields the desired $\Lambda_{n}(\mathrm{p}) \geq\left(2 D^{\mathrm{p}-1} r_{\max }\right)^{-1} R_{n}(\mathrm{p})$.

We now borrow from [26] the concept of extremal p-co-length, coming from generalizing the form of topological max-flow/min-cut duality introduced in [29] for $\mathrm{p}=2$. Thus, alongside the family of paths $\Gamma_{n}$, we consider the family $\Gamma_{n}^{*}$ of all subsets $\gamma^{*} \subset \mathcal{V}_{n}$ that separate $\mathcal{A}_{n}$ from $\mathcal{B}_{n}$, i.e., $\gamma^{*} \in \Gamma_{n}^{*}$ iff any path connecting $\mathcal{A}_{n}$ to $\mathcal{B}_{n}$ contains a vertex in $\gamma^{*}$. Note that $\gamma^{*}$ need not be a path. The extremal p-co-length of $\Gamma_{n}^{*}$ is

$$
\Lambda_{n}^{*}(\mathrm{p}):=\max _{\mathrm{m}} \frac{l_{\mathrm{p}}^{*}\left(\Gamma_{n}^{*}, \mathrm{~m}\right)^{\mathrm{p}^{*}}}{\|\mathrm{~m}\|_{\mathrm{p}}^{\mathrm{p}}}
$$

where $\mathrm{m}: \mathcal{V}_{n} \rightarrow[0, \infty)$ is a weight and $l_{\mathrm{p}}^{*}\left(\Gamma_{n}^{*}, \mathrm{~m}\right):=\min _{\gamma^{*} \in \Gamma_{n}^{*}} \sum_{v \in \gamma^{*}} \mathrm{~m}(v)^{\mathrm{p} / \mathrm{p}^{*}}$ is called the m-co-length of $\Gamma_{n}^{*}$.

By an argument based on Hölder inequality (Theorem 2.2.3 in [26]), the following duality inequality holds for any $\mathrm{m}_{1}, \mathrm{~m}_{2}: \mathcal{V}_{n} \rightarrow[0, \infty)$ :

$$
l_{\mathrm{p}}\left(\Gamma_{n}, \mathrm{~m}_{1}\right) l_{\mathrm{p}}^{*}\left(\Gamma_{n}^{*}, \mathrm{~m}_{2}\right) \leq\left\|\mathrm{m}_{1}\right\|_{\mathrm{p}}\left\|\mathrm{m}_{2}\right\|_{\mathrm{p}}^{\mathrm{p} / \mathrm{p}^{*}} .
$$

Maximizing (once over $\mathrm{m}_{1}$ and once over $\mathrm{m}_{2}$ ), further yields

$$
\begin{aligned}
\Lambda_{n}(\mathrm{p})^{1 / \mathrm{p}} & \leq\left(\frac{\|\mathrm{m}\|_{\mathrm{p}}^{\mathrm{p}}}{l_{\mathrm{p}}^{*}\left(\Gamma_{n}^{*}, \mathrm{~m}\right)^{\mathrm{p}^{*}}}\right)^{1 / \mathrm{p}^{*}}, \\
\Lambda_{n}^{*}(\mathrm{p})^{1 / \mathrm{p}^{*}} & \leq\left(\frac{\|\mathrm{m}\|_{\mathrm{p}}^{\mathrm{p}}}{l_{\mathrm{p}}\left(\Gamma_{n}, \mathrm{~m}\right)^{\mathrm{p}}}\right)^{1 / \mathrm{p}},
\end{aligned}
$$

valid for for any non-zero $\mathrm{m}: \mathcal{V}_{n} \rightarrow[0, \infty)$. Clearly, the inequalities turn to equalities exactly when m realizes the max in the definitions of $\Lambda_{n}(\mathrm{p})$ and $\Lambda_{n}^{*}(\mathrm{p})$, respectively. In particular, we have the duality relation (paralleling (2.13))

$$
\Lambda_{n}(\mathrm{p})^{1 / \mathrm{p}} \Lambda_{n}^{*}(\mathrm{p})^{1 / \mathrm{p}^{*}}=1
$$


Proof of the right inequality in (3.1). Consider a flow $\mathcal{J}$ from $\mathcal{A}_{n}$ to $\mathcal{B}_{n}$ with flux $J(\mathcal{J})=J_{0}>0$. Define $\mathrm{m}: \mathcal{V}_{n} \rightarrow[0, \infty)$ by

$$
\mathrm{m}(v):=\sum_{e \in \mathcal{E}_{n}: v \in \partial e}|\mathcal{J}(e)|^{\mathrm{p}^{*} / \mathrm{p}} .
$$

We first claim that

$$
l_{\mathrm{p}}^{*}\left(\Gamma_{n}^{*}, \mathrm{~m}\right) \geq \frac{1}{D} J_{0}
$$

Fix $\gamma^{*} \in \Gamma_{n}^{*}$ and consider vertices reachable from $\mathcal{A}_{n}$ without crossing $\gamma^{*}$ :

$$
\mathcal{V}_{\mathcal{A}}:=\left\{v \in \mathcal{V}_{n}: \exists \text { a path } \alpha \text { with vertices in } \mathcal{V}_{n} \backslash \gamma^{*} \text { joining } \mathcal{A}_{n} \text { to } v\right\} \text {. }
$$

Clearly, $\mathcal{A}_{n} \subset \mathcal{V}_{\mathcal{A}}$, and $\mathcal{B}_{n} \cap \mathcal{V}_{\mathcal{A}}=\emptyset$ because $\gamma^{*}$ separates. Thus $\sum_{v \in \mathcal{V}_{\mathcal{A}}} \operatorname{div} \mathcal{J}(v)=$ $\sum_{v \in \mathcal{A}_{n}} \operatorname{div} \mathcal{J}(v)=-J_{0}$. At the same time, by cancelling the contributions from edges with both ends in $\mathcal{V}_{\mathcal{A}}$, we get

$$
\begin{aligned}
J_{0}=\sum_{v \in \mathcal{V}_{\mathcal{A}}}-\operatorname{div} \mathcal{J}(v) & =\sum_{e: e_{-} \in \mathcal{V}_{\mathcal{A}}} \mathcal{J}(e)-\sum_{e: e_{+} \in \mathcal{V}_{\mathcal{A}}} \mathcal{J}(e) \\
& =\sum_{e: e_{-} \in \mathcal{V}_{\mathcal{A}}, e_{+} \notin \mathcal{V}_{\mathcal{A}}} \mathcal{J}(e)-\sum_{e: e_{+} \in \mathcal{V}_{\mathcal{A}}, e_{-} \notin \mathcal{V}_{\mathcal{A}}} \mathcal{J}(e) \\
& =\sum_{e: e_{-} \in \mathcal{V}_{\mathcal{A}}, e_{+} \in \gamma^{*}} \mathcal{J}(e)-\sum_{e: e_{+} \in \mathcal{V}_{\mathcal{A}}, e_{-\in \gamma^{*}}} \mathcal{J}(e) \\
& \leq \sum_{e: \partial e \cap \gamma^{*} \neq \emptyset}|\mathcal{J}(e)|,
\end{aligned}
$$

where we also used that if $e_{-} \in \mathcal{V}_{\mathcal{A}}$ and $e_{+} \notin \mathcal{V}_{\mathcal{A}}$ then $e_{+} \in \gamma^{*}$ (or else we could connect from $\mathcal{A}_{n}$ to $e_{+}$, putting $e_{+}$in $\left.\mathcal{V}_{\mathcal{A}}\right)$. The above inequality allows one to estimate:

$$
\begin{aligned}
\sum_{v \in \gamma^{*}} \mathrm{~m}(v)^{\mathrm{p} / \mathrm{p}^{*}} & =\sum_{v \in \gamma^{*}}\left(\sum_{e: v \in \partial e}|\mathcal{J}(e)|^{\mathrm{p}^{*} / \mathrm{p}}\right)^{\mathrm{p} / \mathrm{p}^{*}} \geq \sum_{v \in \gamma^{*}} \frac{1}{D} \sum_{e: v \in \partial e}|\mathcal{J}(e)| \\
& =\frac{1}{D} \sum_{e: \partial e \cap \gamma^{*} \neq \emptyset}|\mathcal{J}(e)| \geq \frac{1}{D} J_{0}
\end{aligned}
$$

where we used $\left(t_{1}+\ldots+t_{D}\right)^{r} \geq \frac{1}{D}\left(t_{1}^{r}+\ldots+t_{D}^{r}\right)$ for any $t_{1}, \ldots, t_{D}, r>0$. The claimed inequality (3.13) follows by taking infimum over $\gamma^{*} \in \Gamma_{n}^{*}$.

Second, we majorize the $\mathrm{p}$-norm of $\mathrm{m}$ (in a way similar to (3.5)):

$$
\begin{aligned}
\sum_{v \in \mathcal{V}_{n}} \mathrm{~m}(v)^{\mathrm{p}} & =\sum_{v}\left(\sum_{e: v \in \partial e}|\mathcal{J}(e)|^{\mathrm{p}^{*} / \mathrm{p}}\right)^{\mathrm{p}} \leq \sum_{v} D^{\mathrm{p}-1} \sum_{e: v \in \partial e}\left(|\mathcal{J}(e)|^{\mathrm{p}^{*} / \mathrm{p}}\right)^{\mathrm{p}} \\
& \leq D^{\mathrm{p}-1} \sum_{v} \sum_{e: v \in \partial e}|\mathcal{J}(e)|^{\mathrm{p}^{*}} \leq 2 D^{\mathrm{p}-1} \sum_{e}|\mathcal{J}(e)|^{\mathrm{p}^{*}}=2 D^{\mathrm{p}-1} r_{\min }^{-\mathrm{p}^{*} / \mathrm{p}} P(\mathcal{J}) .
\end{aligned}
$$

Thus, via (3.11), (3.13) and (3.16) yield

$$
\begin{aligned}
\Lambda_{n}(\mathrm{p})=\Lambda_{n}^{*}(\mathrm{p})^{-\mathrm{p} / \mathrm{p}^{*}} & =\inf _{\mathrm{m}}\left(\frac{\|\mathrm{m}\|_{\mathrm{p}}^{\mathrm{p}}}{l_{\mathrm{p}}^{*}\left(\Gamma_{n}^{*}, \mathrm{~m}\right)^{\mathrm{p}^{*}}}\right)^{\mathrm{p} / \mathrm{p}^{*}} \leq\left(\frac{2 D^{\mathrm{p}-1} r_{\min }^{-\mathrm{p}^{*} / \mathrm{p}} P(\mathcal{J})}{\left(\frac{1}{D} J_{0}\right)^{\mathrm{p}^{*}}}\right)^{\mathrm{p} / \mathrm{p}^{*}} \\
& =\frac{2^{\mathrm{p}-1} D^{\mathrm{p}^{2}-\mathrm{p}+1}}{r_{\min }} \frac{P(\mathcal{J})^{\mathrm{p} / \mathrm{p}^{*}}}{J_{0}^{\mathrm{p}}}
\end{aligned}
$$


Minimizing over all $\mathcal{J}$ yields the desired $\Lambda_{n}(\mathrm{p}) \leq \frac{2^{\mathrm{p}-1} D^{\mathrm{p}^{2}-\mathrm{p}+1}}{r_{\min }} R_{n}(\mathrm{p})$

\section{Sup-multiplicativity (Th. 1.3)}

Our goal is to show the left inequality in Theorem 1.3:

$$
R_{n+m}(\mathrm{p}) \geq \alpha(\mathrm{p})^{-\mathrm{p}} R_{n}(\mathrm{p}) R_{m}(\mathrm{p}) \quad(\forall m, n \in \mathbf{N})
$$

where $\alpha(\mathrm{p})>0$ is explicitly identified. By topological duality relation (1.21) (shown in Proposition 2.5), (4.1) is equivalent to

$$
R_{n+m}^{*}(\mathrm{p}) \leq \alpha(\mathrm{p})^{\mathrm{p}^{*}} R_{n}^{*}(\mathrm{p}) R_{m}^{*}(\mathrm{p}) .
$$
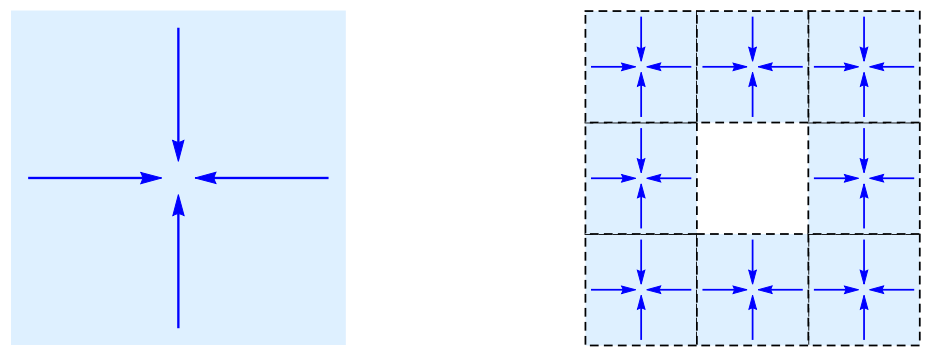

Figure 4.9. Tile substitution rule $\Phi: T_{\text {ini }}$ (left) $\mapsto \Phi\left(T_{\text {ini }}\right)$ (right).

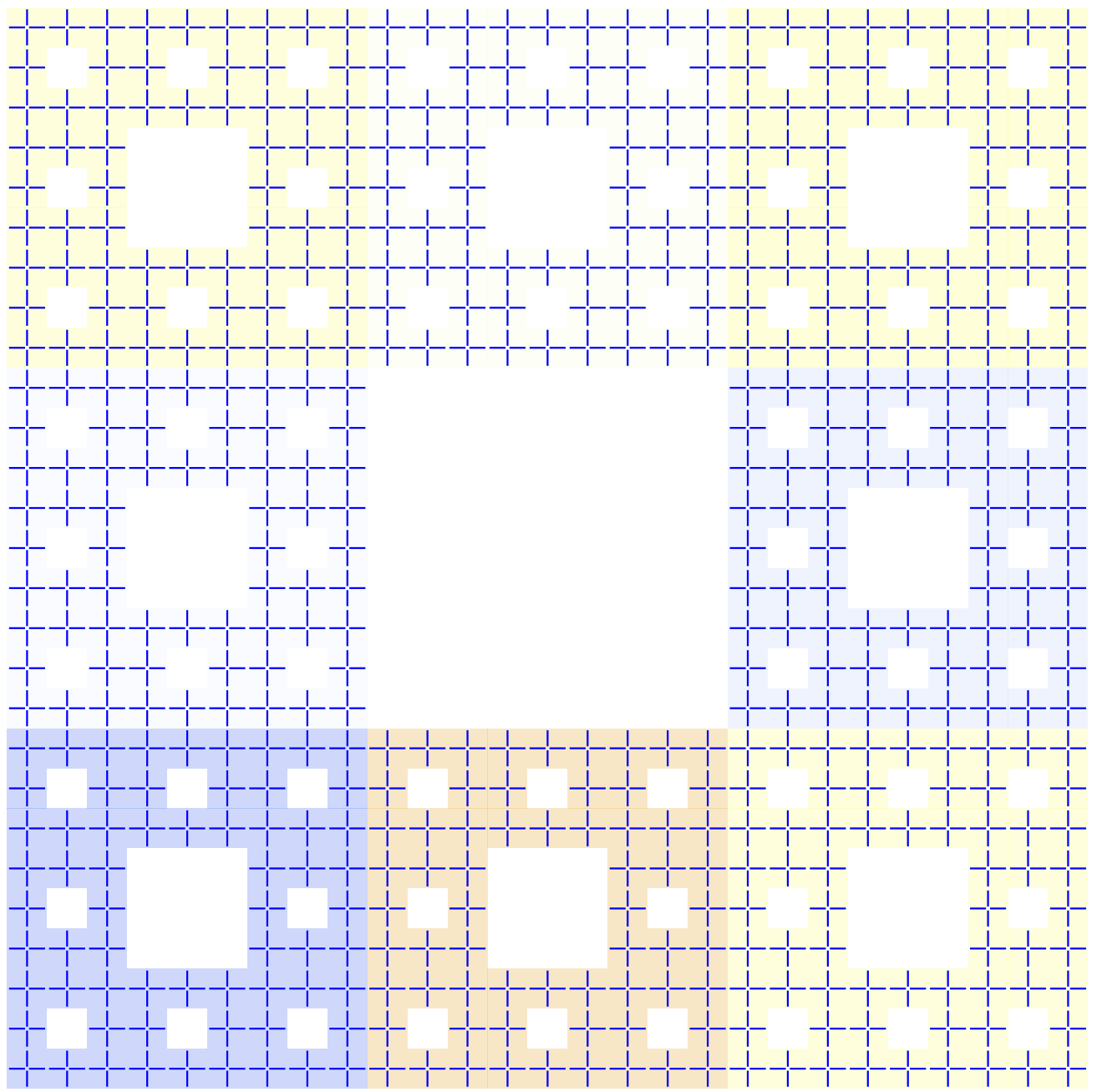

Figure 4.10. $\mathcal{G}_{3}^{*}$ is carried by the tiling $\mathcal{T}_{3}=\Phi^{3}\left(T_{\text {ini }}\right)=\Phi^{2}\left(\mathcal{T}_{1}\right)$, a union of eight 2-supertiles (one for each tile of $\mathcal{T}_{1}$ ). Each 2 -supertile is carrying an imprinted copy of $\mathcal{G}_{2}^{*}$. 
$R_{n}^{*}(\mathrm{p})$ is the resistance of the marked network $\left(\mathcal{G}_{n}^{*}, \mathcal{A}_{n}^{*}, \mathcal{B}_{n}^{*}\right)$ dual to the standard network $\left(\mathcal{G}_{n}, \mathcal{A}_{n}, \mathcal{B}_{n}\right)$. The dual networks were introduced in subsection 1.8 but here we give their precise recursive definition. To make the recursion as simple as possible we will actually produce slightly different networks, still denoted by $\mathcal{G}_{n}^{*}$, that are equivalent (in the sense of subsection 2.4).

We generate $\mathcal{G}_{n}^{*}$ by a simple tile substitution rule $\Phi$ whereby an initial unit-square proto-tile $T_{\text {ini }}$ carrying a cross-wire made of four unit resistors is replaced by its 8 copies, translated and scaled down by $\frac{1}{3}$, as depicted in Figure 4.9. (We leave the formal definition of $\Phi$ to a diligent reader.) Applying the rule $\Phi$ tile-by-tile (with suitable translations and scaling) allows one to iterate and obtain a sequence of tilings, $\Phi\left(T_{\text {ini }}\right), \Phi^{2}\left(T_{\text {ini }}\right):=\Phi\left(\Phi\left(T_{\text {ini }}\right)\right), \ldots$, where the $n$-th term $\mathcal{T}_{n}:=\Phi^{n}\left(T_{\text {ini }}\right)$ is a collection of $8^{n}$ essentially disjoint square tiles covering the $n$th approximant $X_{n}$. The individual tiles in $\mathcal{T}_{n}$ are $3^{-n} \times 3^{-n}$-squares, but (depending on taste) one could well keep them at constant $1 \times 1$ size and scale $X_{n}$ by $3^{n}$ instead.

$\mathcal{G}_{n}^{*}$ is defined as the union of the resistors carried by the tiles of $\mathcal{T}_{n}$. (The input/output sets $\mathcal{A}_{n}^{*}$ and $\mathcal{B}_{n}^{*}$ are still made of the leftmost and rightmost vertices, respectively.) Figure 4.10 depicts thus produced $\mathcal{G}_{n}^{*}$ for $n=3$. By discarding the zero-current edges with ends along the upper and lower boundaries and by joining pairs of serial resistors, this network is equivalent to the network $\mathcal{G}_{n}^{*}$ discussed in the introduction (Figure 1.6), justifying the overloading of the notation. Crucially for our future considerations, because $\Phi^{n+m}\left(T_{\text {ini }}\right)=\Phi^{m}\left(\mathcal{T}_{n}\right)$, the tiling $\mathcal{T}_{n+m}$ is a union of $8^{n}$ copies of $\mathcal{T}_{m}$, each of the form $T^{\prime}=\Phi^{m}(T)$ where $T$ is a tile of $\mathcal{T}_{n}$. We refer to such $T^{\prime}$ as $m$-super-tiles. (2-supertiles are colored in Figure 4.10.)

To start the proof of (4.2), fix $n, m \in \mathbf{N}$. Let $\mathcal{J}$ be the current in $\left(\mathcal{G}_{n}^{*}, \mathcal{A}_{n}^{*}, \mathcal{B}_{n}^{*}\right)$ with flux $J_{0}>0$. The crux is the following lemma, where $\left.\mathcal{J}^{\prime}\right|_{T^{\prime}}$ stands for the restriction of $\mathcal{J}^{\prime}$ to the resistors associated to $T^{\prime}$ (and $\left.\mathcal{J}\right|_{T}$ is in the same vain). We also set $R_{m}^{*}:=R_{m}^{*}(\mathrm{p})$ to streamline formulas.

Lemma 4.1. There is a unit flux flow $\mathcal{J}^{\prime}$ on $\mathcal{G}_{n+m}^{*}$ such that, for every $m$ supertile $T^{\prime}=\Phi^{m}(T)$,

$$
P^{*}\left(\left.\mathcal{J}^{\prime}\right|_{T^{\prime}}\right) \leq \alpha(\mathrm{p})^{\mathrm{p}} R_{m}^{* \mathrm{p} / \mathrm{p}^{*}} P^{*}\left(\left.\mathcal{J}\right|_{T}\right)
$$

with explicit $\alpha(\mathrm{p})>0$, independent of $m, n$ and continuous in $\mathrm{p}>1$.

Assuming the lemma, one readily estimates the total power by summing over all $m$-supertiles:

$$
P^{*}\left(\mathcal{J}^{\prime}\right)=\sum_{T \in \mathcal{T}_{n}} P^{*}\left(\left.\mathcal{J}^{\prime}\right|_{T^{\prime}}\right) \leq \sum_{T \in \mathcal{T}_{n}} \alpha(\mathrm{p})^{\mathrm{p}} R_{m}^{* \mathrm{p} / \mathrm{p}^{*}} P^{*}\left(\left.\mathcal{J}\right|_{T}\right)=\alpha(\mathrm{p})^{\mathrm{p}} R_{m}^{*} \mathrm{p} / \mathrm{p}^{*} P^{*}(\mathcal{J})
$$

This gives the desired inequality (4.2) as follows:

$$
R_{m+n}^{*} \leq \frac{P^{*}\left(\mathcal{J}^{\prime}\right)^{\mathrm{p}^{*} / \mathrm{p}}}{J_{0}^{\mathrm{p}^{*}}} \leq \alpha(\mathrm{p})^{\mathrm{p}^{*}} R_{m}^{*} \frac{P^{*}(\mathcal{J})^{\mathrm{p}^{*} / \mathrm{p}}}{J_{0}^{\mathrm{p}^{*}}}=\alpha(\mathrm{p})^{\mathrm{p}^{*}} R_{m}^{*} R_{n}^{*} .
$$

It remains to prove Lemma 4.1, including identification of $\alpha(\mathrm{p})$.

Proof of Lemma 4.1. In what follows, by a flow in $\mathcal{G}_{m}^{*}$ we mean any vectorfield on $\mathcal{G}_{m}^{*}$, denoted for a moment by $\mathcal{J}_{\circ}: \mathcal{E}_{m}^{*} \rightarrow \mathbf{R}$, that has vanishing divergence at all the vertices not on the boundary of $X_{m}$ and satisfies boundary conditions (1.16) associated to the super-conducting islands. Note that the variant of $\mathcal{G}_{m}^{*}$ we are working with has resistors terminating on all four sides of $X_{m}$. We allow $\mathcal{J}_{\circ}$ to have non-zero fluxes through each side: the left, bottom, right, and top side; and we record 
those fluxes (in that order) as a vector

$$
\mathbf{f}\left(\mathcal{J}_{\circ}\right)=\left(x_{1}, x_{2}, x_{3}, x_{4}\right) \in \mathbf{R}^{4},
$$

called the flux vector of $\mathcal{J}_{\circ}$. (Positive flux indicates inflow.)

Still keeping $m \in \mathbf{N}$ fixed, let $\mathcal{J}_{1010}$ be the unit current from left to right in $\mathcal{G}_{m}^{*}$, so $\mathbf{f}\left(\mathcal{J}_{1010}\right)=(1,0,-1,0)$. By reflecting the portion of $\mathcal{G}_{m}^{*}$ under the diagonal $(y=x)$ in its symmetry line (the anti-diagonal $y=1-x$ ), $\mathcal{J}_{1010}$ yields a unit flux flow $\mathcal{J}_{1100}$ from left to bottom in $\mathcal{G}_{m}^{*}$, i.e., $\mathbf{f}\left(\mathcal{J}_{1100}\right)=(1,-1,0,0)$. (Figure 5.11 depicts the flows for $m=1$.) Up to the flow reversal operation, there are four more counterparts of $\mathcal{J}_{1010}$ and $\mathcal{J}_{1100}$ under the symmetry group of the carpet: $\mathcal{J}_{0101}, \mathcal{J}_{0110}, \mathcal{J}_{0011}$, and $\mathcal{J}_{1001}$, with flux vectors $(0,1,0,-1),(0,1,-1,0),(0,0,1,-1)$, and $(1,0,0,-1)$, respectively. We shall refer to the flows in the set

$$
\mathfrak{J}=\left\{\mathcal{J}_{1010}, \mathcal{J}_{1100}, \mathcal{J}_{0101}, \mathcal{J}_{0110}, \mathcal{J}_{0011}, \mathcal{J}_{1001}\right\}
$$

as pure replacement flows. By construction, they all have the same power

$$
P_{m}^{*}:=P^{*}\left(\mathcal{J}_{\circ}\right)=R_{m}^{* \mathrm{p} / \mathrm{p}^{*}} \quad\left(\mathcal{J}_{\circ} \in \mathfrak{J}\right) .
$$

A simple but crucial property that will enable assembly of replacement flows into a well defined flow $\mathcal{J}^{\prime}$ on $\mathcal{G}_{n+m}^{*}$ is that different $\mathcal{J}_{\circ} \in \mathfrak{J}$ match across their sides (with non-zero flux). Precisely, if we orient such a side and label the $3^{m}$ vertices sitting on it by $1, \ldots, 3^{m}$ (in their natural order), then there are numbers $\mathcal{J}_{\partial}(k)$ $\left(k=1, \ldots, 3^{m}\right)$-independent of $\mathcal{J}_{\circ} \in \mathfrak{J}$-so that, the current through vertex $k$ is equal to $\pm \mathcal{J}_{\partial}(k)$. (Due to symmetry, the orientation choice of the side is immaterial, as $\mathcal{J}_{\partial}\left(3^{m}-k\right)=\mathcal{J}_{\partial}(k)$.)

Capitalizing on the fact that the network $\mathcal{G}_{n+m}^{*}$ is a union of $8^{n}$ copies of $\mathcal{G}_{m}^{*}$, each associated to an $m$-super-tile, the plan is to construct the unit flux flow $\mathcal{J}^{\prime}$ in $\mathcal{G}_{n+m}^{*}$ by replacing the flow $\left.\mathcal{J}\right|_{T}$ in the copy of $\mathcal{G}_{0}^{*}$ associated to tile $T$ (a cross-wire of four resistors) with a new flow $\mathcal{J}^{\prime T^{\prime}}$ in the copy of $\mathcal{G}_{m}^{*}$ associated to the $m$-super-tile $T^{\prime}=\Phi^{m}(T)$.

To describe how the replacement flow $\mathcal{J}^{\prime T^{\prime}}$ is constructed, fix a tile $T$ and let $\mathbf{x}=\left(x_{1}, x_{2}, x_{3}, x_{4}\right)$ be the flux vector of $\left.\mathcal{J}\right|_{T}$. (The $x_{i}$ are flow rates in the cross-wire, so the dot product $\mathbf{x} \cdot \mathbf{1}=0$ where $\mathbf{1}:=(1,1,1,1)$, and the power dissipated in $T$ is $\|\mathbf{x}\|_{\mathrm{p}}^{\mathrm{p}}:=\sum_{i}\left|x_{i}\right|^{\mathrm{p}}$.) We are seeking the replacement flow $\mathcal{J}^{\prime T^{\prime}}$ as a linear combination of the pure replacement flows:

$$
\mathcal{J}^{\prime T^{\prime}}=\sum_{\mathcal{J}_{\circ} \in \mathfrak{J}} t\left(\mathcal{J}_{\circ}\right) \mathcal{J}_{\circ}
$$

where the six parameters $\left(t\left(\mathcal{J}_{\circ}\right)\right)_{\mathcal{J}_{\circ} \in \mathfrak{J}} \in \mathbf{R}^{\mathfrak{J}}$ are chosen so that the flux vectors of $\mathcal{J}^{\prime T^{\prime}}$ and $\left.\mathcal{J}\right|_{T}$ coincide:

$$
\mathbf{x}=\sum_{\mathcal{J}_{\circ} \in \mathfrak{J}} t\left(\mathcal{J}_{\circ}\right) \mathbf{f}\left(\mathcal{J}_{\circ}\right)
$$

For a moment, we assume that such a choice is always possible - as will be evident after inspecting the matrix (4.14) ahead. Observe that the above condition (4.9) ensures that the replacement flows $\mathcal{J}^{\prime T^{\prime}}$ (constructed as above for all supertiles $T^{\prime}$ ) glue into a single well defined flow $\mathcal{J}^{\prime}$ on $\mathcal{G}_{n+m}^{*}$. Indeed, if $T$ and $\tilde{T}$ are adjacent tiles of $\mathcal{T}_{n}$ then they have the same flux of $\mathcal{J}$ - equal to one of the $x_{i}$ associated to tile $T$-through their common side. Therefore, at each individual vertex of the side common to the $m$-supertiles $T^{\prime}$ and $\tilde{T}^{\prime}$, the two replacement flows coincide on the 
two resistors meeting at the vertex - specifically, (4.9) implies that, at the $k$ th vertex (when viewed from $T^{\prime}$ ), the flow equals $x_{i} \mathcal{J}_{\partial}(k)$.

It remains to select the mixing parameters $\mathbf{t}:=\left(t\left(\mathcal{J}_{\circ}\right)\right)_{\mathcal{J}_{\circ} \in \mathfrak{J}}$ in $(4.8)$, which, of course, is best done by minimizing the ratio of powers between $\left.\mathcal{J}\right|_{T}$ and its replacement $\left.\mathcal{J}^{\prime}\right|_{T^{\prime}}$ :

$$
\rho^{*}(\mathbf{x}):=\min \left\{\frac{P^{*}\left(\sum_{\mathcal{J}_{\circ} \in \mathfrak{J}} t\left(\mathcal{J}_{\circ}\right) \mathcal{J}_{\circ}\right)}{\sum_{i=1}^{4}\left|x_{i}\right|^{p}}: \sum_{\mathcal{J}_{\circ} \in \mathfrak{J}} t\left(\mathcal{J}_{\circ}\right) \mathbf{f}\left(\mathcal{J}_{\circ}\right)=\mathbf{x}, \mathbf{t}=\left(t\left(\mathcal{J}_{\circ}\right)\right) \in \mathbf{R}^{\mathfrak{J}}\right\} .
$$

With this choice

$$
P^{*}\left(\left.\mathcal{J}^{\prime}\right|_{T^{\prime}}\right) \leq \rho^{*}(\mathbf{x}) P^{*}\left(\left.\mathcal{J}\right|_{T}\right)
$$

and, to conclude the proof, it remains to bound the power ratio $\rho^{*}(\mathbf{x})$ uniformly in $m$ and $\mathbf{x}$. Specifically, to obtain (4.3), we have to show the inequality

$$
\rho^{*}(\mathbf{x})^{1 / \mathrm{p}} \leq \alpha(\mathrm{p}) R_{m}^{* 1 / \mathrm{p}^{*}}=\alpha(\mathrm{p}) P_{m}^{* 1 / \mathrm{p}} \quad\left(\forall \mathbf{x} \in \mathbf{R}^{4}: \mathbf{x} \cdot \mathbf{1}=0\right) .
$$

This is easiest - next section will make sharper estimates - by invoking Minkowski's ineqaulity for the p-norm (while remembering (4.7)):

$$
\begin{aligned}
\rho^{*}(\mathbf{x})^{1 / \mathrm{p}} & \leq \max _{\mathbf{x}} \min _{\mathbf{t}} \frac{\sum_{\mathcal{J}_{0} \in \mathfrak{J}}\left|t\left(\mathcal{J}_{\circ}\right)\right| P^{*}\left(\mathcal{J}_{\circ}\right)^{1 / \mathrm{p}}}{\left(\sum_{i=1}^{4}\left|x_{i}\right|^{\mathrm{p}}\right)^{1 / \mathrm{p}}} \\
& =\left(\max _{\mathbf{x}} \min _{\mathbf{t}} \frac{\sum_{\mathcal{J}_{\circ} \in \mathfrak{J}}\left|t\left(\mathcal{J}_{\circ}\right)\right|}{\left(\sum_{i=1}^{4}\left|x_{i}\right|^{\mathrm{p}}\right)^{1 / \mathrm{p}}}\right) P_{m}^{* 1 / \mathrm{p}} .
\end{aligned}
$$

To secure (4.12), $\alpha(\mathrm{p})$ can be taken to be the max-min in the parentheses above. To clearly express $\alpha(\mathrm{p})$ and stress that it no longer involves the flows, set $D:=\# \mathfrak{J}=6$, rename the parameters $\mathbf{t}=\left(t\left(\mathcal{J}_{\circ}\right)\right)_{\mathcal{J}_{\circ} \in \hat{\mathcal{J}}}$ and flux vectors $\mathbf{f}=\left(\mathbf{f}\left(\mathcal{J}_{\circ}\right)\right)_{\mathcal{J}_{\circ} \in \hat{\mathcal{J}}}$ as $\mathbf{t}=$ $\left(t_{j}\right)_{j=1}^{D}$ and $\mathbf{f}=\left(\mathbf{f}_{j}\right)_{j=1}^{D}$, respectively. Hence, treated as a matrix with columns $\mathbf{f}_{j}, \mathbf{f}$ becomes

$$
\mathbf{f}=\left(\begin{array}{cccccc}
1 & 1 & 0 & 0 & 0 & 1 \\
0 & -1 & 1 & 1 & 0 & 0 \\
-1 & 0 & 0 & -1 & 1 & 0 \\
0 & 0 & -1 & 0 & -1 & -1
\end{array}\right)
$$

Then our $\alpha(\mathrm{p})$ is given by a constrained max-min problem in $\mathbf{R}^{4} \times \mathbf{R}^{D}$ :

$$
\alpha(\mathrm{p}):=\max _{\mathbf{x}: \mathbf{x} \cdot \mathbf{1}=0} \min _{\mathbf{t}: \sum_{j} t_{j} \mathbf{f}_{j}=\mathbf{x}} \frac{\sum_{j=1}^{D}\left|t_{j}\right|}{\left(\sum_{i=1}^{4}\left|x_{i}\right|^{\mathrm{p}}\right)^{1 / \mathbf{p}}}=\max _{\mathbf{x}:\|\mathbf{x}\|_{\mathbf{p}}=1, \mathbf{x} \cdot \mathbf{1}=0 \quad \mathbf{t}: \sum_{j} t_{j} \mathbf{f}_{j}=\mathbf{x}} \sum_{j=1}^{D}\left|t_{j}\right| .
$$

We note that $\alpha(\mathrm{p})$ is a finite real number because the equation $\sum_{j} t_{j} \mathbf{f}_{j}=\mathbf{x}$ can always be solved given $\mathbf{x}$ with $\mathbf{x} \cdot \mathbf{1}=0$, as can be seen by inspecting the matrix $\mathbf{f}$. Clearly, $\alpha(\mathrm{p})$ is independent of $m$ and positive. That it depends continuously on $\mathrm{p}$ is a routine exercise.

\section{Upper bounds (Th. 1.1)}

As explained in subsection 1.5, upper bounds for $\operatorname{dim}_{A R}(X)$ (including the one given in Theorem 1.1) can be obtained by computing the constant $\alpha(\mathrm{p})$ in the supmultiplicative estimate (4.1) but, for better bounds, one can directly estimate resistance ratios and show $\sup _{n} R_{n+m}^{*}(\mathrm{p}) / R_{n}^{*}(\mathrm{p})<1$ for a specific $m \in \mathbf{N}$. This task, which we undertake in this section, will boil down to estimating the power ratio between the 
current $\mathcal{J}$ in $\mathcal{G}_{n}^{*}$ and its replacement flow $\mathcal{J}^{\prime}$ in $\mathcal{G}_{n+m}^{*}$, as constructed in the proof of Lemma 4.1.

First, reaching back to $(4.10)$, denote by $\rho_{m}^{*(\text { rep })}(\mathrm{p})$ the maximal power ratio under the replacement of a current in $\mathcal{G}_{0}^{*}$ by a current in $\mathcal{G}_{m}^{*}$ (with the same side fluxes),

$$
\rho_{m}^{*(\mathrm{rep})}(\mathrm{p}):=\max _{\mathbf{x} \in \mathbf{R}^{4}: \mathbf{x} \cdot \mathbf{1}=0} \rho^{*}(\mathbf{x}) .
$$

Proposition 5.1. For $m \in \mathbf{N}$, $\operatorname{dim}_{A R}(X) \leq \mathrm{p}$ if

$$
\rho_{m}^{*(\operatorname{rep})}(\mathrm{p})<1
$$

Proof. Summing over all tiles (as in (4.4)), inequality (4.11) gives

$$
P^{*}\left(\mathcal{J}^{\prime}\right) \leq \rho_{m}^{*(\text { rep })}(\mathrm{p}) P^{*}(\mathcal{J}) .
$$

Because $\mathcal{J}$ is a current in $\mathcal{G}_{n}^{*}$ and $\mathcal{J}^{\prime}$ is a flow in $\mathcal{G}_{n+m}^{*}$ (with the same flux), this yields the following inequality between the resistances

$$
R_{n+m}^{*}(\mathrm{p}) \leq \rho_{m}^{*(\mathrm{rep})}(\mathrm{p})^{\mathrm{p}^{*} / \mathrm{p}} R_{n}^{*}(\mathrm{p}) \quad(n \in \mathbf{N}) .
$$

Hence, $\rho_{m}^{*(\text { rep })}(\mathrm{p})<1$ guarantees $\lim \inf _{n \rightarrow \infty} R_{n}^{*}(\mathrm{p})=0$. By duality, $\lim \sup _{n \rightarrow \infty} R_{n}(\mathrm{p})$ $=\infty$, and the bound follows via Theorem 1.2.

Continuing preliminary remarks, if the replacement flow is one of the pure replacement flows in $\mathfrak{J}$ (cf. (4.6)), e.g., when $\mathbf{x}=(1,0,-1,0)$, the associated power ratio $\rho^{*}((1,0,-1,0))$ is what we call (dual) pure power ratio, denoted by $\rho_{m}^{*}(\mathrm{p})$. Clearly, $\rho_{m}^{*}(\mathrm{p}) \leq \rho_{m}^{*(\mathrm{rep})}(\mathrm{p})$. Additionally, by recalling (4.7) and using the duality, we have

$$
\rho_{m}^{*}(\mathrm{p}):=\frac{P_{m}^{*}}{P_{0}^{*}}=\left(\frac{R_{m}^{*}(\mathrm{p})}{R_{0}^{*}(\mathrm{p})}\right)^{\mathrm{p} / \mathrm{p}^{*}}=\left(\frac{R_{m}(\mathrm{p})}{R_{0}(\mathrm{p})}\right)^{-1}=\rho_{m}(\mathrm{p})^{-\mathrm{p} / \mathrm{p}^{*}},
$$

where $\rho_{m}(\mathrm{p}):=P_{m} / P_{0}=\left(R_{m}(\mathrm{p}) / R_{0}(\mathrm{p})\right)^{\mathrm{p}^{*} / \mathrm{p}}$ is the (primary) pure power ratio, the counterpart of $\rho_{m}^{*}(\mathrm{p})$ on the other side of the topological duality - which we plotted in subsection 1.4. (Keep in mind that $R_{0}^{*}(\mathrm{p})=2^{\mathrm{p}^{*} / \mathrm{p}}$-from two serial unit resistorsand $P_{0}^{*}=2$ and $R_{0}(\mathrm{p})=1 / 2$-from two parallel unit resistors.)

Because $\rho_{m}^{*}(\mathrm{p})$ (or $\rho_{m}(\mathrm{p})$ ) does not require maximization over $\mathbf{x}$ and thus is easier to compute than $\rho_{m}^{*(\mathrm{rep})}(\mathrm{p})$, the following technical theorem plays an important role in easing the numerical workload and improving sharpness of our upper bounds.

Theorem 5.2. For any $m \in \mathbf{N}$ and $\mathrm{p} \in(1,2)$,

$$
\rho_{m}^{*(\mathrm{rep})}(\mathrm{p})=\rho_{m}^{*}(\mathrm{p}) \text {. }
$$

Corollary 5.3. For $\mathrm{p} \in(1,2)$,

$$
R_{n+m}^{*}(\mathrm{p}) \leq 2^{-\mathrm{p}^{*} / \mathrm{p}} R_{n}^{*}(\mathrm{p}) R_{m}^{*}(\mathrm{p}) \quad(m, n \in \mathrm{N}) .
$$

In particular, the sup-multiplicativity in Theorem 1.3 holds with $\alpha(\mathrm{p})=2^{-1 / \mathrm{p}}$.

Proof of Corollary 5.3. In view of Theorem 5.2 (coupled with $(5.5)$ and $R_{0}^{*}(\mathrm{p})=$ $\left.2^{\mathrm{p}^{*} / \mathrm{p}}\right)$, inequality (5.4) reads:

$$
R_{n+m}^{*}(\mathrm{p}) \leq \rho_{m}^{*}(\mathrm{p})^{\mathrm{p}^{*} / \mathrm{p}} R_{n}^{*}(\mathrm{p})=\frac{R_{m}^{*}(\mathrm{p})}{R_{0}^{*}(\mathrm{p})} R_{n}^{*}(\mathrm{p})=2^{-\mathrm{p}^{*} / \mathrm{p}} R_{n}^{*}(\mathrm{p}) R_{m}^{*}(\mathrm{p}) .
$$

To recognize that $\alpha(\mathrm{p})=2^{-1 / \mathrm{p}}$, revisit $(4.2)$.

Postponing the proof of Theorem 5.2, we turn to the resulting upper bounds (including the one in subsection 1.2). They were found by computing the zeros of 
$\ln \rho_{m}(\mathrm{p})=-\frac{\mathrm{p}}{\mathrm{p}^{*}} \ln \rho_{m}^{*}(\mathrm{p})$ as a function of $\mathrm{p} \in(1,2)$ (Figure 1.4), which we located with $10^{-8}$ precision and rounded up to the nearest $10^{-6}$ (cf. Section 8).

\section{Proposition 5.4.}

$$
\begin{array}{ll}
\operatorname{dim}_{A R}(X) \leq 1.847596 & (m=1), \\
\operatorname{dim}_{A R}(X) \leq 1.830543 & (m=2), \\
\operatorname{dim}_{A R}(X) \leq 1.821203 & (m=3), \\
\operatorname{dim}_{A R}(X) \leq 1.815778 & (m=4), \\
\operatorname{dim}_{A R}(X) \leq 1.812292 & (m=5), \\
\operatorname{dim}_{A R}(X) \leq 1.809864 & (m=6), \\
\operatorname{dim}_{A R}(X) \leq 1.808076 & (m=7), \\
\operatorname{dim}_{A R}(X) \leq 1.806703 & (m=8) .
\end{array}
$$

Proof of Proposition 5.4 (computer assisted). Fix one of the listed $\mathrm{p}$ (and the corresponding $m$ ). Via Proposition 5.1 and Theorem 5.2, it suffices to show $\rho_{m}^{*}(\mathrm{p})<1$. To do this we computed a flow $\mathcal{J}_{1010}^{\text {approx }}$ that is an approximation to the pure replacement current $\mathcal{J}_{1010}$. From $(5.5), \rho_{m}^{*}(\mathrm{p}) \leq P_{\text {approx }}^{*} / 2$ where $P_{\text {approx }}^{*}:=P^{*}\left(\mathcal{J}_{1010}^{\text {approx }}\right)$. The proof is ended by evaluating $P_{\text {approx }}^{*}$ with sufficient numerical precision to guarantee $P_{\text {approx }}^{*} / 2<1$.

Remark 5.5. One could speculate (cf. Section 6) whether an analogous current replacement scheme with power ratio $\rho_{m}(\mathrm{p})=P_{m} / P_{0}$ exists on the primary side of topological duality, i.e., between $\mathcal{G}_{n}$ and $\mathcal{G}_{n+m}$. In such case, for any $m \in \mathbf{N}$, the common zero of $\ln \rho_{m}^{*}(\mathrm{p})$ and $\ln \rho_{m}(\mathrm{p})$ would be equal to $\operatorname{dim}_{A R}(X)$ (since arbitrary small perturbations of $\mathrm{p}$ would yield both $\rho_{m}(\mathrm{p}) \overline{<1}$ and $\left.\rho_{m}^{*}(\mathrm{p})<1\right)$. However, the zeros visibly vary with $m$ (see Figure 1.4 ), so such scheme cannot exist.
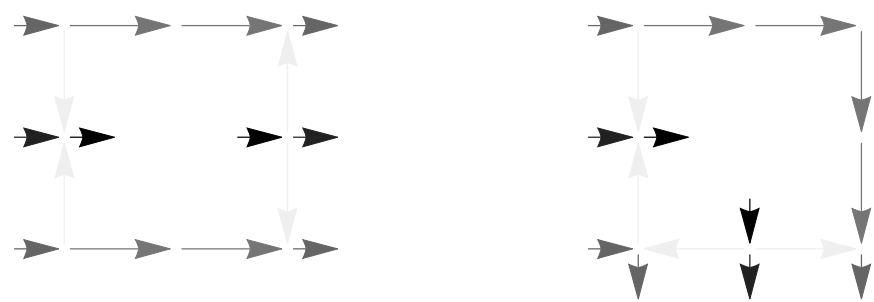

Figure 5.11. Approximations to pure replacement flows $\mathcal{J}_{1010}^{\text {approx }}$ (left) and $\mathcal{J}_{1100}^{\text {approx }}$ (right) for parameters $\mu=0.2041$ and $\delta=0.0332$ (at $\mathrm{p}=1.8477$ and $m=1$ ). In $J_{1010}^{\text {approx }}$, the incoming flow rates (indicated by shading) are: $1 / 2-\mu$ in the upper/lower resistors and $2 \mu$ in the middle resistor; $2 \mu+2 \delta$ through the superconducting center island (not depicted), and $1 / 2-\mu-\delta$ in the resistors above/below the island.

Proof of the upper bound in Theorem 1.1. This bound coincides with the one given by Proposition 5.4 for $m=1$. We treat it separately because, in this case, all computations can be tracked by hand, yielding a fully rigorous human verifiable proof. To shorten our decimals let us round up to $\mathrm{p}=1.8477$.

First, we find a good unit flux flow $\mathcal{J}_{1010}^{\text {approx }}$ in $\mathcal{G}_{1}^{*}$. Because of symmetry, we look among the flows as depicted on the left of Figure 5.11, which are parametrized by just two real parameters $\mu$ and $\delta$. We compute half of the total power dissipated in 
the 24 unit resistors with non-zero current to be

$$
\frac{P_{\text {approx }}^{*}}{2}=2|1 / 2-\mu|^{\mathrm{p}}+4|1 / 2-\mu-\delta|^{\mathrm{p}}+4|\delta|^{\mathrm{p}}+|2 \mu|^{\mathrm{p}}+|2 \mu+2 \delta|^{\mathrm{p}} \approx 0.999885<1,
$$

where the approximation is for specific values $\mu=0.2041$ and $\delta=0.0332$ at $\mathrm{p}=$ 1.8477. This $\mathrm{p}$ was selected so that the minimum of $\frac{P_{\mathrm{approx}}^{*}}{2}$ over $\mu$ and $\delta$ is just shy of 1 , in a way that is guaranteed by the precision of the numerical evaluation of $\frac{P_{\text {approx }}^{*}}{2}$. $\left(\frac{P_{\mathrm{approx}}^{*}}{2}\right.$ is a convex function of $(\mu, \delta)$; Figure 5.12 depicts it near the unique minimum $(\mu, \beta) \approx(0.2041,0.0332)$.)

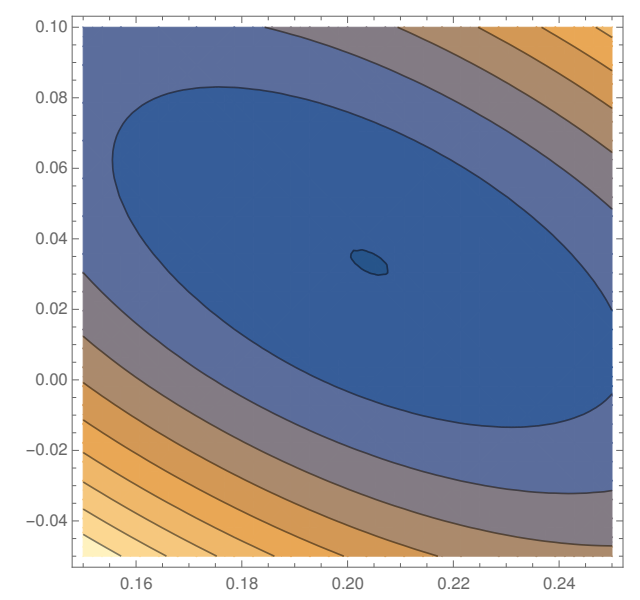

Figure 5.12. Contours of the power of the flow $\mathcal{J}_{1010}^{\text {approx }}$ as a function of parameters $(\mu, \delta)$; note a unique minimum $(\mu, \delta) \approx(0.2041,0.0332)$. $(\mathrm{p}=1.8477$ and $m=1$.

It remains to prove Theorem 5.2. The argument depends on closer analysis of the replacement flows $\mathcal{J}^{\prime T^{\prime}}$, defined by (4.8) as a linear combination of the six pure replacement flows in $\mathfrak{J}$. Because we fix the $m$-supertile $T^{\prime}$ and identify its network with $\mathcal{G}_{m}^{*}$, let us strike the superscript $T^{\prime}$ and simplify $\mathcal{J}^{\prime T^{\prime}}$ to $\mathcal{J}^{\prime}$ (not to be confused with the flow $\mathcal{J}^{\prime}$ on $\mathcal{G}_{n+m}^{*}$ we spoke about before).

To start, let the vector field $F_{1}: \mathcal{E}_{m}^{*} \rightarrow \mathbf{R}$ come from restricting the pure replacement flow $\mathcal{J}_{1010}$ to the left triangle, by which we mean the edges of $\mathcal{G}_{m}^{*}$ contained in the region given by $|x| \geq|y|, x \leq 0$ in the translated coordinate system with origin at the center of $X$. On the edges not in the triangle we set $F_{1}$ to zero, so $F_{1}$ is not a flow on $\mathcal{G}_{m}^{*}$ as it has non-zero divergence along the diagonal sides of the triangle (with flux $\frac{1}{2}$ through each side). Likewise, let $T_{2}$ be a similar restriction of $\mathcal{J}_{1010}$ to the bottom triangle (with fluxes $\frac{1}{2}$ and $-\frac{1}{2}$ through its two diagonal sides and none through its base). Further, let $F_{2}, F_{3}, F_{4}$ be the counterclockwise rotations of $F_{1}$ by $90^{\circ}, 180^{\circ}, 270^{\circ}$; and let $T_{1}, T_{3}, T_{4}$ be the rotations of $T_{2}$ by $270^{\circ}, 90^{\circ}, 180^{\circ}$. (See Figure 5.13.) Note that $\mathcal{J}_{1010}$ can be decomposed as $\mathcal{J}_{1010}=F_{1}-F_{3}+T_{2}-T_{4}$, and all other pure replacement flows in the sextuple $\mathfrak{J}$ can be decomposed similarly.

Lemma 5.6. (Structure of replacement flows) Given $\mathbf{x} \in \mathbf{R}^{4}$ with $\mathbf{x} \cdot \mathbf{1}=0$, if $\mathcal{J}^{\prime}$ is a linear combination of the pure replacement flows (i.e., equals $\mathcal{J}^{\prime T^{\prime}}$ in (4.8)) and has flux vector $\mathbf{x}$ (i.e., (4.9) holds) then, for some $\kappa \in \mathbf{R}$,

$$
\mathcal{J}^{\prime}=\sum_{i=1}^{4} x_{i} F_{i}+\sum_{i=1}^{4}\left(\frac{x_{i-1}-x_{i+1}}{2}+\kappa\right) T_{i}
$$

where the addition of the indices is $\bmod 4$ (i.e. $4+1=1$ and $1-1=4$ ). Moreover, any $\mathcal{J}^{\prime}$ as above is a linear combination of the pure replacement flows in $\mathfrak{J}$. 


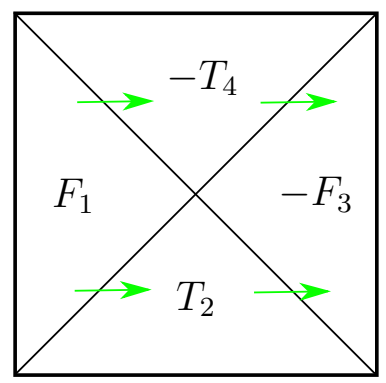

$\mathcal{J}_{1010}$

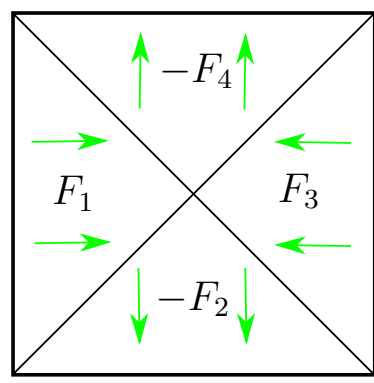

$\mathcal{J}_{1111}$

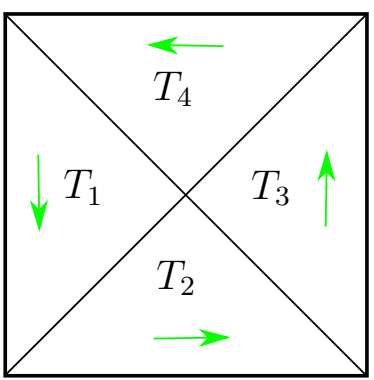

$\mathcal{J}_{0000}$

Figure 5.13. Pure $\mathcal{J}_{1010}$, saddle $\mathcal{J}_{1111}$, and vortex $\mathcal{J}_{0000}$ flows decomposed into vector fields $F_{i}$ and $T_{i}$. (Arrows indicate the general direction of the flow.)

Proof of Lemma 5.6. Clearly, $\mathcal{J}^{\prime}$ is a linear combination $\mathcal{J}^{\prime}=\sum_{i=1}^{4} a_{i} F_{i}+b_{i} T_{i}$ (for some $a_{i}, b_{i} \in \mathbf{R}$ ) because this is so for each pure replacement flow. The fluxes of $\mathcal{J}^{\prime}$ through the sides are $x_{i}$ and they come from the $a_{i} F_{i}$, so $a_{i}=x_{i}$ (for $i=$ $1, \ldots, 4)$. The coefficients $b_{i}$ are determined by vanishing of divergence along the boundaries of the triangles - amounting to balancing of fluxes between the triangles. For instance, consider the flux from the left triangle to the top triangle across the half-diagonal $y=-x, x \leq 0$. In the flow $\mathcal{J}^{\prime}, a_{1} F_{1}, a_{4} F_{4}, b_{1} T_{1}$, and $b_{4} T_{4}$ meet along this half-diagonal so balancing of fluxes dictates $a_{1} \frac{1}{2}+a_{4} \frac{1}{2}+b_{1}\left(-\frac{1}{2}\right)+b_{4} \frac{1}{2}=0$. Thus $b_{1}-b_{4}=a_{1}+a_{4}=x_{1}+x_{4}$. By considering all four half-diagonals, we get four continuity equations:

$$
b_{i+1}-b_{i}=x_{i+1}+x_{i} \quad(i=1,2,3,4)
$$

which solve to

$$
b_{i}=\frac{1}{2}\left(x_{i-1}-x_{i+1}\right)+\kappa \quad(\kappa \in \mathbf{R}) .
$$

(To verify that these are solutions: $2\left(b_{i+1}-b_{i}\right)=x_{i}-x_{i+2}-x_{i-1}+x_{i+1}=2 x_{i+1}+2 x_{i}$ since $-x_{i+2}-x_{i-1}=x_{i}+x_{i+1}$.) This proves (5.7).

It remains to see that $\mathcal{J}^{\prime}$ given by (5.7) is a linear combination of pure replacement flows. To do this (and for future reference), let us introduce two special replacement flows: saddle flow $\mathcal{J}_{1111}$ and vortex flow $\mathcal{J}_{0000}$ - see Figure 5.13 - defined by

$$
\begin{aligned}
& \mathcal{J}_{1111}:=F_{1}-F_{2}+F_{3}-F_{4}=\frac{1}{2}\left(\mathcal{J}_{1100}-\mathcal{J}_{0110}+\mathcal{J}_{0011}+\mathcal{J}_{1001}\right) \\
& \mathcal{J}_{0000}:=T_{1}+T_{2}+T_{3}+T_{4}=\frac{1}{2}\left(-\mathcal{J}_{1100}-\mathcal{J}_{0110}-\mathcal{J}_{0011}+\mathcal{J}_{1001}\right)
\end{aligned}
$$

with the verification of the equalities left for the reader. One then checks that $\mathcal{J}^{\prime}$ in (5.7) can be expressed as follows

$$
\mathcal{J}^{\prime}=\frac{x_{1}-x_{3}}{2} \mathcal{J}_{1010}-\frac{x_{4}-x_{2}}{2} \mathcal{J}_{0101}+\frac{x_{1}+x_{3}}{2} \mathcal{J}_{1111}+\kappa \mathcal{J}_{0000},
$$

making it a linear combination of the six pure replacement flows.

Conclusion of proof of Theorem 5.2: In view of the definition (5.1) of $\rho_{m}^{*(\mathrm{rep})}(\mathrm{p})$ and $\rho_{m}^{*}(\mathrm{p})=\frac{P_{m}^{*}}{2}($ from $(5.5))$, the task is to show that, for any fixed $\mathbf{x} \in \mathbf{R}^{4}$ with $\mathbf{x} \cdot \mathbf{1}=0$, we have $\rho^{*}(\mathbf{x}) \leq \frac{P_{m}^{*}}{2}$. This, in turn, amounts to showing existence of a choice of the linear combination in (4.8) forming the replacement current $\mathcal{J}^{\prime}:=\mathcal{J}^{\prime T^{\prime}}$ 
with flux vector $\mathbf{x}$ so that

$$
\frac{P^{*}\left(\mathcal{J}^{\prime}\right)}{\sum_{i=1}^{4}\left|x_{i}\right|^{\mathrm{p}}} \leq \frac{P_{m}^{*}}{2}
$$

By (5.7), $\mathbf{x}$ determines $\mathcal{J}^{\prime}$ up to one parameter $\kappa \in \mathbf{R}$, and we set $\kappa:=0$.

The power $P^{*}\left(\mathcal{J}^{\prime}\right)$ is the sum of the powers contributed by individual edges, organized into disjoint orbits under the dihedral symmetry group $D_{4}$ of $X$. To fix attention, let us consider a single edge $e$ in the bottom half of the left triangle. Assume first that $e$ is not contained in the $x$-axis, in which case its orbit consist of eight edges. Set $e_{1}:=e$ and let $e_{8}$ be the image of $e$ under the reflection in the $x$-axis. Moreover let $e_{2}, e_{3}$ and $e_{4}, e_{5}$ and $e_{6}, e_{7}$ be the images of $e_{8}, e_{1}$ under counterclockwise rotations by $90^{\circ}, 180^{\circ}, 270^{\circ}$, respectively. Because $F_{1}\left(e_{1}\right)=F_{1}\left(e_{8}\right)$ and $T_{1}\left(e_{1}\right)=-T_{1}\left(e_{8}\right)$, the power contributed by the pair $e_{1}, e_{8}$ is

$$
\left|\mathcal{J}^{\prime}\left(e_{1}\right)\right|^{\mathrm{p}}+\left|\mathcal{J}^{\prime}\left(e_{8}\right)\right|^{\mathrm{p}}=\left|x_{1} F_{1}(e)+\frac{x_{4}-x_{2}}{2} T_{1}(e)\right|^{\mathrm{p}}+\left|x_{1} F_{1}(e)-\frac{x_{4}-x_{2}}{2} T_{1}(e)\right|^{\mathrm{p}} .
$$

The situation is similar in the three other triangles. Taking into account $F_{1}\left(e_{1}\right)=$ $F_{1}\left(e_{8}\right)=F_{2}\left(e_{2}\right)=F_{2}\left(e_{3}\right)=F_{3}\left(e_{4}\right)=F_{3}\left(e_{5}\right)=F_{4}\left(e_{7}\right)=\ldots$ and $-T_{1}\left(e_{1}\right)=T_{1}\left(e_{8}\right)=$ $T_{2}\left(e_{2}\right)=-T_{2}\left(e_{3}\right)=T_{3}\left(e_{4}\right)=-T_{3}\left(e_{5}\right)=T_{4}\left(e_{6}\right)=\ldots$ the total power contribution from the dihedral orbit of $e$ is

$$
\begin{aligned}
\sum_{j=1}^{8}\left|\mathcal{J}^{\prime}\left(e_{i}\right)\right|^{\mathrm{p}} & =\sum_{i=1}^{4}\left|x_{i} F_{1}(e)+\frac{x_{i-1}-x_{i+1}}{2} T_{1}(e)\right|^{\mathrm{p}}+\left|x_{i} F_{1}(e)-\frac{x_{i-1}-x_{i+1}}{2} T_{1}(e)\right|^{\mathrm{p}} \\
& \leq \sum_{i=1}^{4} 2\left|x_{i} F_{1}(e)\right|^{\mathrm{p}}+2\left|\frac{x_{i-1}-x_{i+1}}{2} T_{1}(e)\right|^{\mathrm{p}} \\
& =2\left|F_{1}(e)\right|^{\mathrm{p}} \sum_{i=1}^{4}\left|x_{i}\right|^{\mathrm{p}}+2\left|T_{1}(e)\right|^{\mathrm{p}} \sum_{i=1}^{4}\left|\frac{x_{i-1}-x_{i+1}}{2}\right|^{\mathrm{p}} \\
& \leq 2\left|F_{1}(e)\right|^{\mathrm{p}} \sum_{i=1}^{4}\left|x_{i}\right|^{\mathrm{p}}+2\left|T_{1}(e)\right|^{\mathrm{p}} \sum_{i=1}^{4}\left|x_{i}\right|^{\mathrm{p}}
\end{aligned}
$$

where the first inequality is an instance of Clarkson's inequality [14, 28]

$$
\|\mathbf{x}+\mathbf{y}\|_{\mathrm{p}}^{\mathrm{p}}+\|\mathbf{x}-\mathbf{y}\|_{\mathrm{p}}^{\mathrm{p}} \leq 2\|\mathbf{x}\|_{\mathrm{p}}^{\mathrm{p}}+2\|\mathbf{y}\|_{\mathrm{p}}^{\mathrm{p}} \quad\left(\mathrm{p} \in(1,2), \mathbf{x}, \mathbf{y} \in \mathbf{R}^{4}\right),
$$

and we also exploited the fact that $\sigma: \mathbf{R}^{4} \rightarrow \mathbf{R}^{4}$ given by $\sigma\left(\left(x_{i}\right)_{i=1}^{4}\right):=\left(x_{i+1}\right)_{i=1}^{4}$ is an isometry of $\|\cdot\|_{p}$ so that

$$
\left\|\sigma^{-1} \mathbf{x}-\sigma \mathbf{x}\right\|_{\mathrm{p}} \leq\left\|\sigma^{-1} \mathbf{x}\right\|_{\mathrm{p}}+\|\sigma \mathbf{x}\|_{\mathrm{p}}=2\|\mathbf{x}\|_{\mathrm{p}}
$$

When $e$ is in the left triangle and on the $x$-axis, its dihedral orbit consist of only four edges $e_{1}:=e, e_{2}, e_{3}, e_{4}$ obtained by iterated rotation by $90^{\circ}$. Because $T_{i}\left(e_{i}\right)=0$, the power contribution of this orbit is

$$
\sum_{j=1}^{4}\left|\mathcal{J}^{\prime}\left(e_{i}\right)\right|^{\mathrm{p}}=\sum_{i=1}^{4}\left|x_{i} F_{i}\left(e_{i}\right)\right|^{\mathrm{p}}=\sum_{i=1}^{4}\left|x_{i} F_{1}(e)\right|^{\mathrm{p}}=\sum_{i=1}^{4}\left|x_{i}\right|^{\mathrm{p}}\left|F_{1}(e)\right|^{\mathrm{p}} .
$$

Compare the right hand sides of (5.14) and (5.17) to the two corresponding contributions of the orbit of $e$ to the power $P_{m}^{*}=P^{*}\left(\mathcal{J}_{1010}\right)$ of the pure current 
$\mathcal{J}_{1010}=F_{1}-F_{3}+T_{2}-T_{4}:$

$$
\sum_{j=1}^{8}\left|\mathcal{J}_{1010}\left(e_{i}\right)\right|^{\mathrm{p}}=4\left|F_{1}\left(e_{1}\right)\right|^{\mathrm{p}}+4\left|T_{1}\left(e_{1}\right)\right|^{\mathrm{p}} \quad \text { and } \quad \sum_{j=1}^{4}\left|\mathcal{J}_{1010}\left(e_{i}\right)\right|^{\mathrm{p}}=2\left|F_{1}\left(e_{1}\right)\right|^{\mathrm{p}} \text {. }
$$

They differ by the factor $\frac{\sum_{i=1}^{4}\left|x_{i}\right|^{\mathrm{P}}}{2}$. Therefore, summing over all orbits yields

$$
P^{*}\left(\mathcal{J}^{\prime}\right) \leq \frac{1}{2} \sum_{i=1}^{4}\left|x_{i}\right|^{\mathrm{p}} P^{*}\left(\mathcal{J}_{1010}\right)=\frac{1}{2} \sum_{i=1}^{4}\left|x_{i}\right|^{\mathrm{p}} P_{m}^{*},
$$

which is the inequality (5.13) we set out to prove.

\section{Sub-multiplicativity (Th. 1.3)}

Our goal is to prove the right inequality in Theorem 1.3:

$$
R_{n+m}(\mathrm{p}) \leq \beta(\mathrm{p})^{\mathrm{p}} R_{n}(\mathrm{p}) R_{m}(\mathrm{p}) \quad(\forall m, n \in \mathbf{N})
$$

where $\beta=\beta(\mathrm{p})>0$ is explicitly identified.
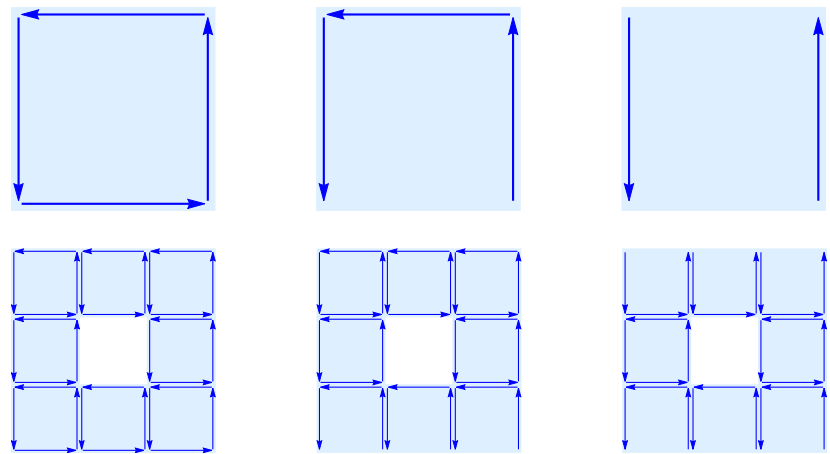

Figure 6.14. Substitution $\Phi_{\text {std }}: T_{A}, T_{B}, T_{C}$ (above) $\mapsto \Phi\left(T_{A}\right), \Phi\left(T_{B}\right), \Phi\left(T_{C}\right)$ (below). All unit resistors. (Network vertices, at the squares' corners, are suppressed.)
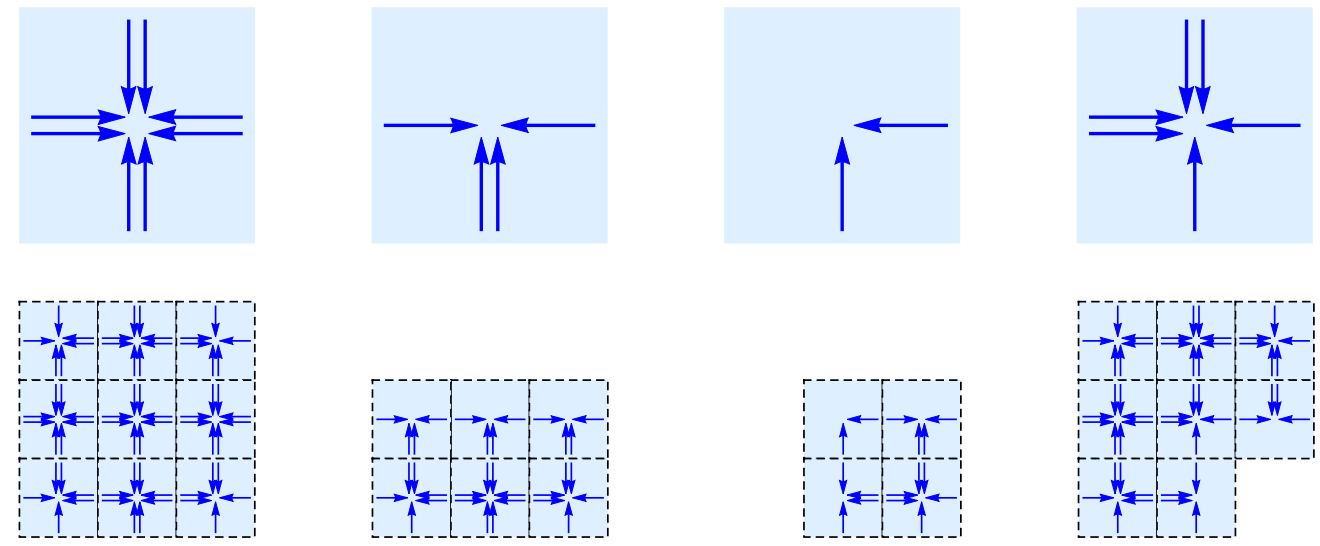

Figure 6.15. Substitution $\Phi: T_{A}, T_{B}, T_{C}, T_{D}$ (above) $\mapsto \Phi\left(T_{A}\right), \Phi\left(T_{B}\right), \Phi\left(T_{C}\right), \Phi\left(T_{D}\right)$ (below). All half-unit resistors (i.e., $r(e)=2^{-\mathrm{p} / \mathrm{p}^{*}}$ ); none on tile edges.

The argument mirrors the proof of (4.2), with the standard network $\mathcal{G}_{n}$ in the place of its dual $\mathcal{G}_{n}^{*}$, but (in view of Remark 5.5) we have to employ a more complex substitution. (Another approach is to use a different network altogether, as we do in the next section.) To start, let us generate $\mathcal{G}_{n}$ via the standard substitution rule $\Phi_{\text {std }}$ with three distinct proto-tiles depicted in Figure 6.14. (Here and elsewhere, we 
consider the proto-tiles up to congruence by Euclidean motions.) We designate prototile $T_{C}$ as the initial proto-tile, which means that we consider the sequence of tilings $\Phi_{\text {std }}^{n}\left(T_{C}\right)$. By merging all pairs of parallel resistors, the marked network carried by the tiling $\Phi_{\text {std }}^{n}\left(T_{C}\right)$ is equivalent to the standard network $\mathcal{G}_{n}$ in Figure 1.3. (Incidentally, this explains the non-unit resistances in $\mathcal{G}_{n}$.) An astute reader will note that, had we allowed the immaterial zero-current edges along the top and the bottom, we could have used a single proto-tile $T_{A}$ and the network of $\Phi_{\text {std }}^{n}\left(T_{A}\right)$. However, dealing with three proto-tiles is a build-up for what follows. (In this section, in all networks, we designate the top and bottom vertices as the input and output sets.) The difficulty to overcome is that $\Phi_{\text {std }}$ is marred by the flaw of having resistors along tile's edges. This means that we can no longer use simple matching of fluxes across sides to secure Kirchhoff's first law when gluing flows on individual tiles (or supertiles) into a global flow (as we did in Section 4).

We remedy the problem by recutting the network into different tiles, as expressed by the new substitution, with four proto-tiles, depicted in Figure 6.15. Individual arrows represent resistors of resistance $2^{-\mathrm{p} / \mathrm{p}^{*}}$ each, which makes them half-unit resistors: two in series give resistance one (per (2.9)). The idea is that four copies of tile $T_{C}$ (rotated by $0^{\circ}, 90^{\circ}, 180^{\circ}, 270^{\circ}$ ) form a tiling, denote it by $T_{C \times 4}$, whose network is equivalent (by joining serial resistors) to that of four unit resistors along the edges of a unit square, a version of $\mathcal{G}_{0}$ including the zero current edges. Thus one should think of $T_{C}$ as carrying a quarter of $\mathcal{G}_{0}$ and of $\Phi$ as substituting the two (half-unit) resistors by sixteen such resistors. The other three proto-tiles carry an even number of resistors that can be split into several pairs, each forming a corner (i.e., meeting at the 90 degree angle). The effect of $\Phi$ on tiles $T_{A}, T_{B}, T_{D}$ amounts to replacing each such corner pair by the network carried by $\Phi\left(T_{C}\right)$, suitably rotated or reflected. E.g., $T_{B}$ carries 4 resistors arranged into two corners, one a reflection of the other in a vertical axis; correspondingly, $\Phi\left(T_{B}\right)$ carries 32 resistors coming as two symmetric copies of those carried by $\Phi\left(T_{C}\right)$. (To avert a possible confusion: $T_{A}$ carries 8 resistors arranged into four corners, each a copy of the network carried by $T_{C}$, but $T_{A}$ is not the same as $T_{C \times 4}$.) Consequently, by an easy induction on $n$, the network carried by $\Phi^{n}\left(T_{C \times 4}\right)$ (see Figure 6.16) is equivalent to $\mathcal{G}_{n}$ (upon joining and merging pairs of resistors and dropping the zero-current edges). In the following arguments, we abuse the notation and still denote this $\Phi$-born network by $\mathcal{G}_{n}$.

Before starting the estimates, consider the decomposition of $\mathcal{G}_{n+m}$ into networks carried by $m$-supertiles $T^{\prime}=\Phi^{m}(T)$ where $T$ is a tile of the tiling $\Phi^{n}\left(T_{C \times 4}\right)$ (Figure 6.16). Although, the network carried by $T^{\prime}$ is no longer a copy of the whole $\mathcal{G}_{m}$, it is a union of several translated copies of $\mathcal{G}_{m-1}$ and halves of $\mathcal{G}_{m-1}$; see Figures 6.17 and 6.18. (This is again proven by induction on $m$.) This will allow us to directly relate $R_{n+m}(\mathrm{p})$ and $R_{m-1}(\mathrm{p})$, which will be enough because $R_{m-1}(\mathrm{p})$ and $R_{m}(\mathrm{p})$ are comparable on the force of the following easy lemma the proof of which is relegated to the end of this section.

Lemma 6.1. For all $m \in \mathbf{N}$, we have $\frac{1}{2} R_{m-1}(\mathrm{p}) \leq R_{m}(\mathrm{p}) \leq 3^{\mathrm{p}-1} R_{m-1}(\mathrm{p})$.

To start the proof of inequality (6.1), fix $m, n \in \mathbf{N}$ and let $\mathcal{J}$ be the unit flux current in $\mathcal{G}_{n}$. We also drop p from $R_{m}$ (p) to streamline formulas. Inequality (6.1) results (along the same lines) from the following analogue of Lemma 4.1, which gives a replacement flow and a bound on its power. 
Lemma 6.2. There is a unit flux flow $\mathcal{J}^{\prime}$ on $\mathcal{G}_{n+m}$ such that, for every $m$ supertile $T^{\prime}=\Phi^{m}(T)$,

$$
P\left(\left.\mathcal{J}^{\prime}\right|_{T^{\prime}}\right) \leq \beta(\mathrm{p})^{\mathrm{p}^{*}} R_{m}^{\mathrm{p}^{*} / \mathrm{p}} P\left(\left.\mathcal{J}\right|_{T}\right)
$$

with explicit $\beta(\mathrm{p})>0$, independent of $m, n$ and continuous in $\mathrm{p}>1$.

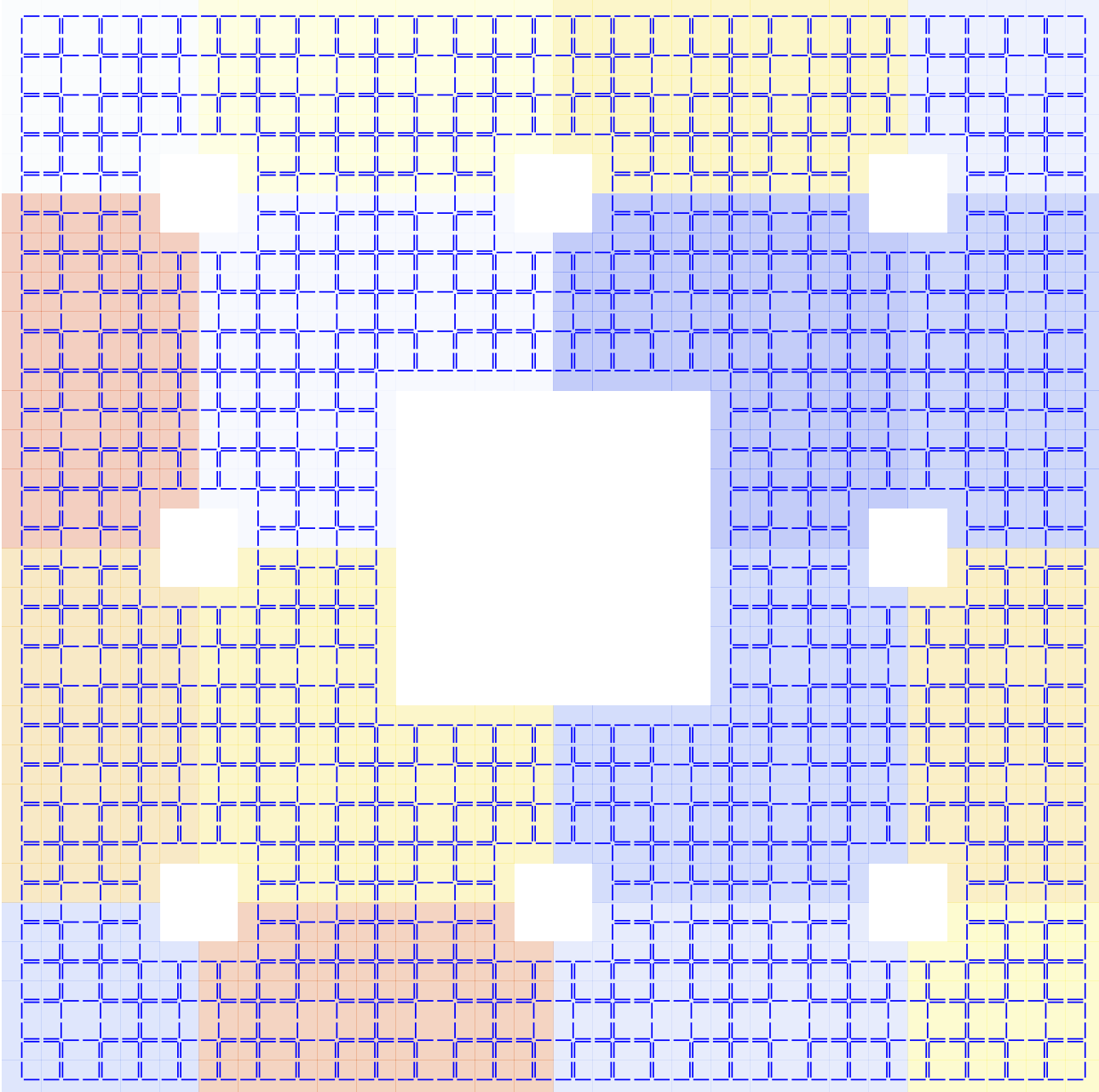

Figure 6.16. The network carried by $\Phi^{3}\left(T_{C \times 4}\right)$. Merging parallel resistors yields $\mathcal{G}_{3}$. (The 2-supertiles are colored.)

Proof of Lemma 6.2. Consider a tile $T$ and the associated $m$-super-tile $T^{\prime}:=$ $\Phi^{m}(T)$. Let $\mathcal{J}_{1}$ be the unit flux current from top to bottom in $\mathcal{G}_{m-1}$. As before, cutting $\mathcal{J}_{1}$ in half along the diagonal and reflecting the upper half in the anti-diagonal, constructs a unit flow $\mathcal{J}_{2}$ in $\mathcal{G}_{m-1}$ from left to bottom. Set $P:=P\left(\mathcal{J}_{1}\right)=P\left(\mathcal{J}_{2}\right)$. Our main task is constructing from $\mathcal{J}_{1}$ and $\mathcal{J}_{2}$ replacement flows on $m$-supertiles.

First, given a flow $\mathcal{J}$ on $\mathcal{G}_{n}$, denote by $\mathbf{f}\left(\left.\mathcal{J}\right|_{T}\right) \in \mathbf{R}^{k}$ the flux vector recording the flow through the individual resistors in $T$ listed in the counterclockwise order of their appearance along the boundary of $T$ starting with the top of the left side of $T$ (and with positive sign indicating inflow). The dimension $k=k_{\tau}$ depends on the tile type $\tau \in\{A, B, C, D\}$. Hence, for type $C$ tile, $\mathbf{f}\left(\left.\mathcal{J}\right|_{T}\right)=(c,-c) \in \mathbf{R}^{2}$ where $c$ is the flow through the bottom resistor. Accordingly, the flow vectors for type $B, D, A$ form linear subspaces of $\mathbf{R}^{4}, \mathbf{R}^{6}, \mathbf{R}^{8}$ of dimensions $3,5,7$, respectively. 
An analogous flux vector $\mathbf{f}\left(\left.\mathcal{J}^{\prime}\right|_{T^{\prime}}\right) \in \mathbf{R}^{k}$, recording the fluxes through the sides of a supertile $T^{\prime}=\Phi^{m}(T)$, can be associated to the restriction of any flow $\mathcal{J}^{\prime}$ on $\mathcal{G}_{n+m}$ to $T^{\prime}$. Here we define the sides of $T^{\prime}$ so that they bijectively correspond to the resistors in $T$ : a side is a segment of the boundary of $T^{\prime}$ spanned by the vertices of one of the half-copies of $\mathcal{G}_{m-1}$ making up the network carried by $T^{\prime}$. In this way, the resistors with vertices on any particular side are the ones generated by $\Phi^{m}$ from a single resistor in $T$. For instance, supertile $\Phi^{m}\left(T_{C}\right)$ - see Figure 6.17 (left)-has two sides: the bottom one and the right one, and $\mathbf{f}\left(\left.\mathcal{J}^{\prime}\right|_{T^{\prime}}\right)=(c,-c) \in \mathbf{R}^{2}$ where $c$ is the flow through the bottom side. (The left and upper edges of $T^{\prime}$ do not count as sides; the flow cannot enter $T^{\prime}$ through them.) The $B, D, A$ supertiles have 4,6,8 sides, respectively - see Figure 6.17 (right) and Figure 6.18.
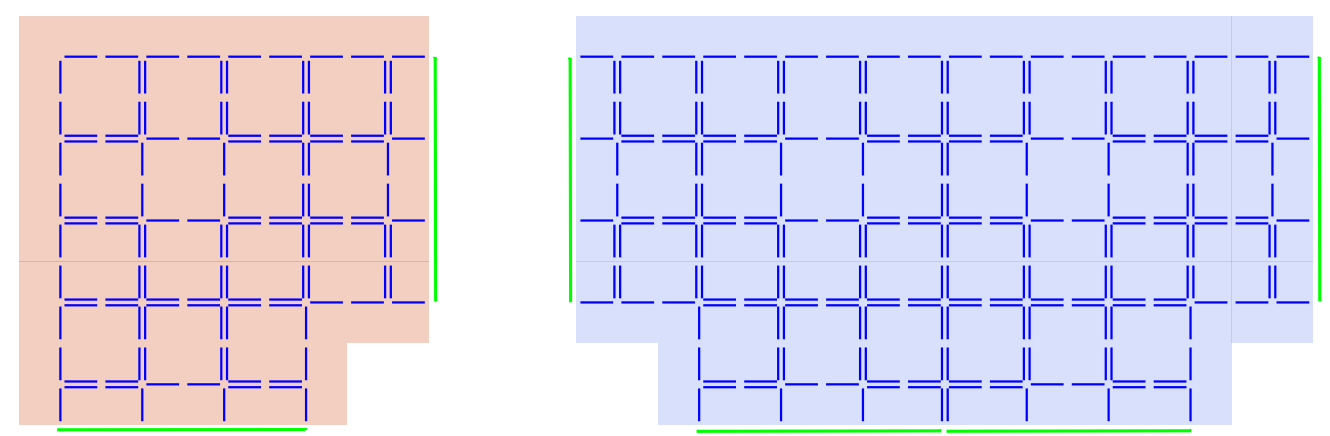

Figure 6.17. 2-supertiles are stitched from copies of $\mathcal{G}_{1}$ and half- $\mathcal{G}_{1}: 2$-supertile $\Phi^{2}\left(T_{C}\right)$ (left) uses $1 \times \mathcal{G}_{1}$ and $2 \times$ half- $\mathcal{G}_{1} ; 2$-supertile $\Phi^{2}\left(T_{B}\right)$ (right) uses $2 \times \mathcal{G}_{1}$ and $4 \times$ half- $\mathcal{G}_{1}$. (Green segments indicate sides. $\Phi^{2}\left(T_{B}\right)$ has four sides.)
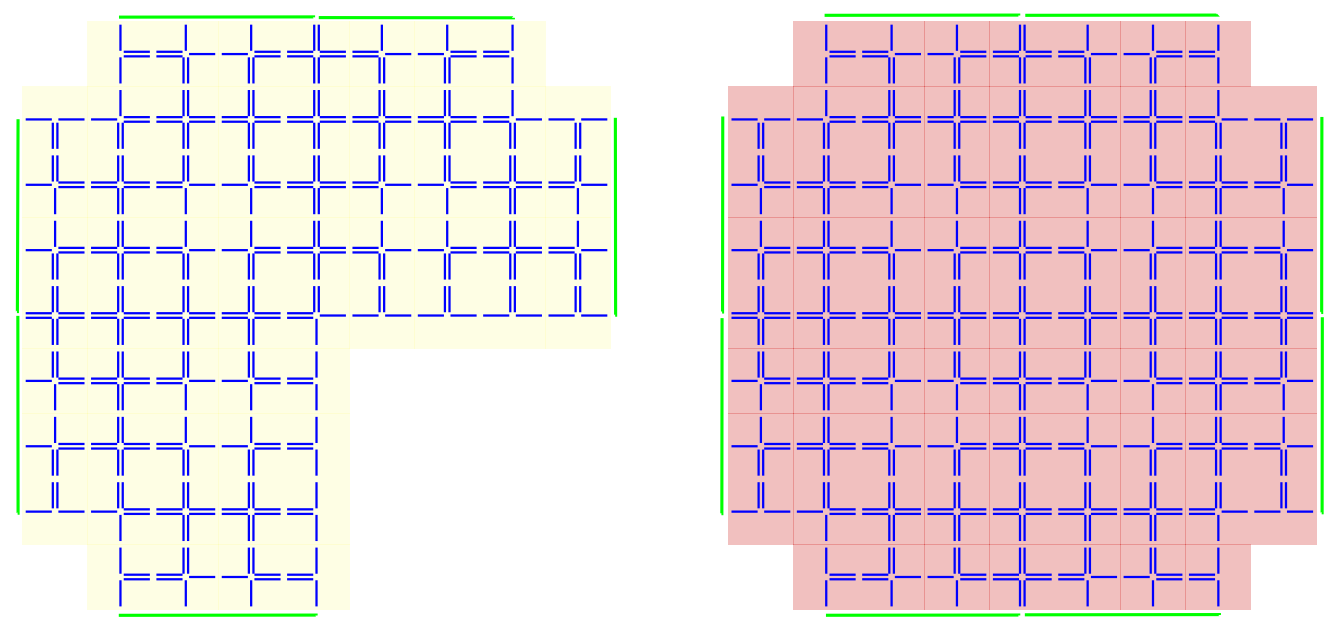

Figure 6.18. 2-supertile $\Phi^{2}\left(T_{D}\right)$ (left) uses $3 \times \mathcal{G}_{1}$ and $6 \times$ half- $\mathcal{G}_{1} 2$-supertile $\Phi^{2}\left(T_{A}\right)$ (right) uses $4 \times \mathcal{G}_{1}$ and $8 \times$ half- $\mathcal{G}_{1}$. (Green segments indicate sides; 6 for $\Phi^{2}\left(T_{D}\right)$ and 8 for $\Phi^{2}\left(T_{A}\right)$.)

The next step is to use multiple copies of currents $\mathcal{J}_{1}$ and $\mathcal{J}_{2}$ in $\mathcal{G}_{m-1}$ and their images under the dihedral symmetry group (and flow reversal) to make pure replacement flows in $m$-super-tiles $T^{\prime}=\Phi^{m}(T)$ with flux vectors that linearly span the space of all possible flux vectors for the given tile type of $T$. For $T=T_{C}$, looking at Figure 6.17 , let $\mathcal{J}_{C}$ be the flow with flux vector $(1,-1)$ obtained by gluing: the bottom half of $\mathcal{J}_{1}$ (reversed so it inflows through the bottom), $\mathcal{J}_{2}$ rotated by $90^{\circ}$, and another bottom half of $\mathcal{J}_{1}$ rotated by $90^{\circ}$. One could call this flow a corner flow. Note that $P\left(\mathcal{J}_{C}\right)=2 P$. For $T=T_{B}$, we will have three pure replacement flows. 
The first two $\mathcal{J}_{B, 1}$ and $\mathcal{J}_{B, 2}$ with flux vectors $(1,-1,0,0)$ and $(0,0,-1,1)$, respectively, are corner flows, using only half of the supertile. The third, with flux vector $(1,0,0,-1)$, is $\mathcal{J}_{B, 3}$ obtained by gluing (suitably rotated): two halves of $\mathcal{J}_{1}$ and two full $\mathcal{J}_{1}$ into a horizontal strip going from left to right, what we call pass-through flow. Note that $P\left(\mathcal{J}_{B, 3}\right)=3 P$. In a similar way, one makes $\mathcal{J}_{D, 1}, \ldots, \mathcal{J}_{D, 5}$ so that their flux vectors are linearly independent (and have entries 0 or \pm 1 ). (E.g., $\mathcal{J}_{D, 1}$ could be the snaking flow with flux $(0,1,0,-1,0,0)$ obtained from two copies of $\mathcal{J}_{2}$, one copy of $\mathcal{J}_{1}$, and two copies of half of $\mathcal{J}_{1}$.) $\mathcal{J}_{A, 1}, \ldots, \mathcal{J}_{A, 7}$ follow suit. The power of any pure replacement flow is bounded by some universal multiple of $P$; by our count, it is $4 P$, the power of the snaking flow.

We finished collecting the needed pure replacement flows $\mathcal{J}_{\tau, i}(\tau \in\{A, B, C, D\}$, $\left.i=1, \ldots, k_{\tau}-1\right)$. Denote by $\mathbf{f}_{\tau, i}:=\mathbf{f}\left(\mathcal{J}_{\tau, i}\right) \in \mathbf{R}^{k_{\tau}}$ their flux vectors. By construction, for any possible tile type $\tau \in\{A, B, C, D\}$ of tile $T$ and any flux vector $\mathbf{x} \in \mathbf{R}^{k_{\tau}}$ of the restricted flow $\left.\mathcal{J}\right|_{T}$, one can linearly combine the pure replacement flows $\mathcal{J}_{\tau, i}$ to get a flow $\mathcal{J}^{\prime T^{\prime}}$ on the $m$-supertile $T^{\prime}=\Phi^{m}(T)$,

$$
\mathcal{J}^{\prime T^{\prime}}=\sum_{i=1}^{k_{\tau}} t_{i} \mathcal{J}_{\tau, i}
$$

with the prescribed flux:

$$
\mathbf{x}=\sum_{i=1}^{k_{\tau}} t_{i} \mathbf{f}_{\tau, i} .
$$

Crucially, the individual flows $\mathcal{J}^{\prime T^{\prime}}$ assemble into a flow on $\mathcal{G}_{n+m}$ by the same argument verifying Kirchhoff's first law at the vertices on boundaries between two $m$-supertiles used in the proof of Lemma 4.1.

Just like we defined $\alpha(\mathrm{p})$ in Section 4 , we take $\tilde{\beta}(\mathrm{p})$ given by

$$
\tilde{\beta}(\mathbf{p}):=\max _{\tau} \max _{\mathbf{x}:\|\mathbf{x}\|_{\mathbf{p}^{*}=1,} \mathbf{x} \cdot \mathbf{1}=0} \min _{\mathbf{t}: \sum_{i} t_{i} \mathbf{f}_{\tau, i}=\mathbf{x}} \sum_{i}\left|t_{i}\right| .
$$

The power ratio is then estimated uniformly in $\mathbf{x}$ by the triangle inequality

$$
\begin{aligned}
\rho(\mathbf{x})^{1 / \mathrm{p}^{*}} & :=\max _{\tau} \min _{\mathbf{t}: \sum_{i} t_{i} \mathbf{f}_{\tau, i}=\mathbf{x}} \frac{P\left(\mathcal{J}^{\prime T^{\prime}}\right)^{1 / \mathrm{p}^{*}}}{P\left(\left.\mathcal{J}\right|_{T}\right)^{1 / \mathrm{p}^{*}}} \\
& \leq \max _{\tau} \max _{\mathbf{x}: \mathbf{x} \cdot \mathbf{1}=0} \min _{\mathbf{t}: \sum_{i} t_{i} \mathbf{f}_{\tau, i}=\mathbf{x}} \frac{\sum_{i}\left|t_{i}\right| P\left(\mathcal{J}_{\tau, i}^{\prime}\right)^{1 / \mathbf{p}^{*}}}{P\left(\left.\mathcal{J}\right|_{T}\right)^{1 / \mathbf{p}^{*}}} \\
& \leq \tilde{\beta}(\mathrm{p}) \max _{\tau, i} P\left(\mathcal{J}_{\tau, i}^{\prime}\right)^{1 / \mathbf{p}^{*}}
\end{aligned}
$$

where we used that $\|\mathbf{x}\|_{\mathbf{p}^{*}}^{\mathrm{p}^{*}}=P\left(\left.\mathcal{J}\right|_{T}\right)$. Thus

$$
\rho(\mathrm{x}) \leq \tilde{\beta}(\mathrm{p})^{\mathrm{p}^{*}} 4 P=\tilde{\beta}(\mathrm{p})^{\mathrm{p}^{*}} 4 R_{m-1}^{\mathrm{p}^{*} / \mathrm{p}}
$$

where we used that $P\left(\mathcal{J}_{\tau, i}^{\prime}\right) \leq 4 P$. Combined with Lemma 6.1 , this gives inequality (6.1) with

$$
\beta(\mathrm{p}):=\tilde{\beta}(\mathrm{p}) 4^{1 / \mathrm{p}^{*}} 2^{1 / \mathrm{p}}=\tilde{\beta}(\mathrm{p}) 2^{1+1 / \mathrm{p}^{*}} .
$$

Proof of Lemma 6.1. We first prove the second inequality, which reads $R_{m}^{*} / R_{m-1}^{*}$ $\geq 1 / 3$ on the other side of the topological duality.

Consider the unit flux current $\mathcal{J}^{\prime}$ in $\mathcal{G}_{m}^{*}$ so that $P_{m}^{*}=P^{*}\left(\mathcal{J}^{\prime}\right)$. Taking $\Phi$ to be the substitution used in Section 4 to generate $\mathcal{G}_{m}^{*}$, one can obtain a flow $\mathcal{J}$ in $\mathcal{G}_{m-1}^{*}$ 
by replacing the network carried by each 1-supertile $T^{\prime}=\Phi(T)$ in $\mathcal{G}_{m}^{*}$ (Figure 4.9, right) with a simple cross-wire of four resistors carried by the tile $T$ (Figure 4.9, left). In order to satisfy Kirchhoff's first law, we collapse the flow in $T^{\prime}$ to one in $T$ in an obvious way: the flows in the four resistors of $T$ are set to the respective side fluxes $x_{i}(i=1,2,3,4)$ of $T^{\prime}$. The power dissipated by $\mathcal{J}$ in $T$ is then $\sum_{i=1}^{4}\left|x_{i}\right|^{\mathrm{p}}$. On the other hand, the power $P^{*}\left(\left.\mathcal{J}^{\prime}\right|_{T^{\prime}}\right)$ dissipated by $\mathcal{J}^{\prime}$ in $T^{\prime}$ can be crudely estimated from below as follows. The current $\mathcal{J}^{\prime}$ in the three resistors attached to the left side of $T^{\prime}$ is of the form $t_{1} x_{1}, t_{2} x_{1}, t_{3} x_{1}$ where $\sum_{j} t_{j}=1$, with the associated power estimated via convexity of $t \mapsto t^{\mathrm{p}}$ :

$$
\sum_{j=1}^{3}\left|t_{j} x_{1}\right|^{\mathrm{p}}=3\left|x_{1}\right|^{\mathrm{p}}\left(\frac{\sum_{j}\left|t_{j}\right|^{\mathrm{p}}}{3}\right) \geq 3\left|x_{1}\right|^{\mathrm{p}}\left|\frac{\sum_{j} t_{j}}{3}\right|^{\mathrm{p}}=\left|x_{1}\right|^{\mathrm{p}} 3^{1-\mathrm{p}} .
$$

By accounting for all four sides, we get $P^{*}\left(\left.\mathcal{J}^{\prime}\right|_{T^{\prime}}\right) \geq \sum_{i=1}^{4}\left|x_{i}\right|^{\mathrm{p}} 3^{1-\mathrm{p}}=3^{1-\mathrm{p}} P^{*}\left(\left.\mathcal{J}\right|_{T}\right)$. Therefore, $P_{m}^{*} / P_{m-1}^{*} \geq 3^{1-\mathrm{p}}$, which amounts to $R_{m}^{*} / R_{m-1}^{*} \geq 3^{\frac{(1-\mathrm{p}) \mathrm{p}^{*}}{\mathrm{p}}}=3^{-1}$.

The other inequality $R_{m} / R_{m-1} \geq \frac{1}{2}$ follows by a similar argument involving potentials on the dual side. Consider a potential $\mathcal{U}^{\prime}$ of the current in $\mathcal{G}_{m}^{*}$ with drop $U_{0}=1$. As before, replace each 1 -supertile with a cross-wire and assign to the center of the cross the potential of the superconducting island, while letting the potential of the arm ends be unchanged (i.e. inherited form the corresponding vertices in $\mathcal{G}_{m}^{*}$ ). This produces a unit drop potential $\mathcal{U}$ on $\mathcal{G}_{m-1}^{*}$. Note that each edge of $\mathcal{E}_{m-1}^{*}$ is an arm of the cross-wire and thus corresponds to two edges $e_{1}, e_{2} \in \mathcal{E}_{m}^{*}$. The respective drops of the potential, $\delta:=\nabla \mathcal{U}(e)$ and $\delta_{j}:=\nabla \mathcal{U}^{\prime}\left(e_{j}\right)(j=1,2)$, satisfy $\delta=\delta_{1}+\delta_{2}$, and their contributions to the power are again related by convexity:

$$
\sum_{j=1}^{2}\left|\delta_{j}\right|^{\mathrm{p}^{*}} \geq 2\left(\frac{1}{2} \sum_{j=1}^{2}\left|\delta_{j}\right|\right)^{\mathrm{p}^{*}} \geq 2^{1-\mathrm{p}^{*}}|\delta|^{\mathrm{p}^{*}}
$$

We conclude that $P^{*}\left(\mathcal{U}^{\prime}\right) \geq 2^{1-\mathrm{p}^{*}} P^{*}(\mathcal{U})$, which gives $R_{m}^{*} \leq 2^{\mathrm{p}^{*}-1} R_{m-1}^{*}$, or $R_{m} \geq$ $2^{-1} R_{m-1}$ on the other side of the duality.

Note that when relating resistances at different approximation levels, one faces a choice to either proceed from a richer network to a simpler network - by reducing, i.e., collapsing a current or restricting a potential, as in Lemma 6.1 - or vice versa-by extending/interpolating, as in Lemma 6.2. Exercising good power control is crucial, and it is more subtle and effective in the latter case. Additional choices in forging an argument come from picking sides of the two dualities (convex and topological).

\section{Lower bounds (Th. 1.1)}

As explained in subsection 1.5, lower bounds for $\operatorname{dim}_{A R}(X)$ (including the one given in Theorem 1.1) are broadly based on the sup-multiplicative estimate (4.1) and come from uniform bounds on the resistance ratios between networks. These boil down to estimating suitable power ratios, as we will do here, paralleling Section 5 (on the other side of topological duality). However, we introduce an important variation by changing the approximating networks. This both simplifies computations and sharpens the results. We abandon the standard graph approximations $\mathcal{G}_{n}$ in favor of networks $\tilde{\mathcal{G}}_{n}$ created by the substitution given in Figure 7.19 , with $T_{A}$ serving as the initial tile. The network $\tilde{\mathcal{G}}_{n}$, carried by $\Phi^{n}\left(T_{A}\right)$, has no resistors touching the top and the bottom and is naturally taken with the left and right vertices as the input 
and output sets, respectively. (This represents $90^{\circ}$ rotation with respect to $\mathcal{G}_{n}$ but matches $\mathcal{G}_{n}^{*}$ and will help us parallel Section 5.)

The key is that the substitution places no resistors along edges of its tiles and is simpler than the one used in the previous section, yet the resistances of the networks are comparable (Lemma 7.1). (In particular, this section gives another proof of submultiplicativity (6.1).) Beyond the use of a different network, the lower bounds (as compared to the upper bounds) are complicated by more difficult estimates of power ratios - due to exponents $\mathrm{p}$ and $\mathrm{p}^{*}$ sitting on the opposite sides of the hilbertian 2 . In particular, pursuing the analogue of Theorem 5.2 (Theorem 7.4), we faced a choice to either considerably weaken the inequality or treat it as a conjecture (Conjecture 7.3), to be numerically verified for every pair of specific $\mathrm{p}$ and $m$ we have to consider. One either has to settle for weaker lower bounds or accept more computer assistance in the proofs. For instance, to show $\operatorname{dim}_{A R}(X)>1+\frac{\ln 2}{\ln 3} \approx 1.6309$, one can either use $\tilde{\mathcal{G}}_{2}$ and skirt the conjecture (Proposition 7.7 ) or use a smaller network $\tilde{\mathcal{G}}_{1}$ and verify the conjecture (see Proof of Theorem 1.1, ahead). (By resistor joining and symmetry, $\tilde{\mathcal{G}}_{2}$ reduces to circa 20 resistors, while $\tilde{\mathcal{G}}_{1}$ reduces to only 4 resistors.)
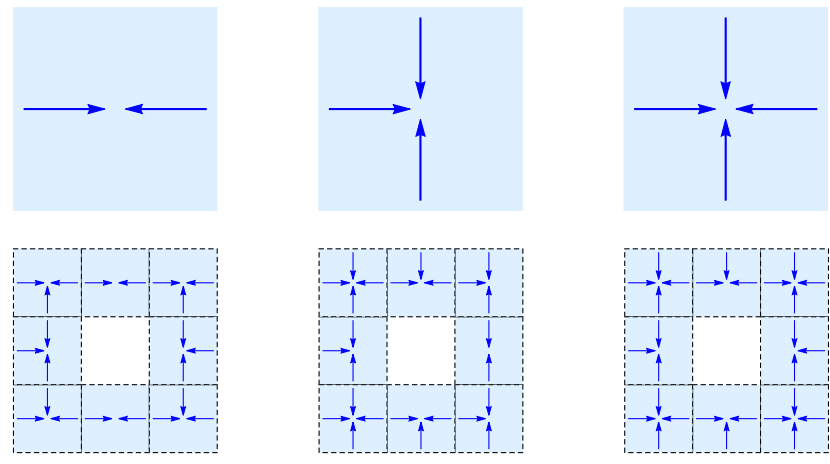

Figure 7.19. Alternative substitution rule $\Phi: T_{A}, T_{B}, T_{C}$ (above) $\mapsto \Phi\left(T_{A}\right), \Phi\left(T_{B}\right), \Phi\left(T_{C}\right)$ (below). The network $\tilde{\mathcal{G}}_{n}$ is carried by $\Phi^{n}\left(T_{A}\right)$.

To start, observe that the network $\tilde{\mathcal{G}}_{n}$ (cf. Figure 7.22) allows for easy gluing of flows, much like $\mathcal{G}_{n}^{*}$ did. One way to see this is to note that $\tilde{\mathcal{G}}_{n}$ is a subnetwork of $\mathcal{G}_{n}^{*}$ obtained by pruning the resistors that connect to the boundaries of the superconducting islands. Therefore, flows in $\tilde{\mathcal{G}}_{n}$ are synonymous with flows $\mathcal{G}_{n}^{*}$ that vanish on those resistors. As a result, we can repeat the constructions in Sections 4 and 5 (with obvious modifications) to construct a scheme whereby a flow $\mathcal{J}$ on $\tilde{\mathcal{G}}_{n}$ is replaced by a flow $\mathcal{J}^{\prime}$ on $\tilde{\mathcal{G}}_{n+m}$ by substituting the restricted flow $\left.\mathcal{J}\right|_{T}$ in the network carried by each tile $T$ with a replacement flow $\mathcal{J}^{\prime T^{\prime}}$ on the network carried by the supertile $T^{\prime}=\Phi^{m}(T)$, while preserving the flux vectors. As before, the replacement flow $\mathcal{J}^{\prime T^{\prime}}$ is obtained as a linear combination of six pure replacement flows in the set

$$
\mathfrak{J}=\left\{\mathcal{J}_{1010}, \mathcal{J}_{1100}, \mathcal{J}_{0101}, \mathcal{J}_{0110}, \mathcal{J}_{0011}, \mathcal{J}_{1001}\right\}
$$

which all have the same power

$$
\tilde{P}_{m}:=P\left(\mathcal{J}_{\circ}\right)=\tilde{R}_{m}(\mathrm{p})^{\mathrm{p}^{*} / \mathrm{p}} \quad\left(\mathcal{J}_{\circ} \in \mathfrak{J}\right) .
$$

The tilde in $\tilde{P}_{m}$ and $\tilde{R}_{m}(\mathrm{p})$ is to avoid confusion with the corresponding powers $P_{m}$ and resistances $R_{m}(\mathrm{p})$ for the standard network; although, the following lemma makes the distinction inconsequential for the value of the critical exponent $\mathrm{p}_{\text {res }}$.

Lemma 7.1. There is a constant $C>0$ such that $\frac{1}{C} \leq \tilde{R}_{n}(\mathrm{p}) / R_{n}(\mathrm{p}) \leq C$ for all $n \in \mathbf{N}$ and $\mathrm{p}>1$. 
Proof. See Appendix 9.
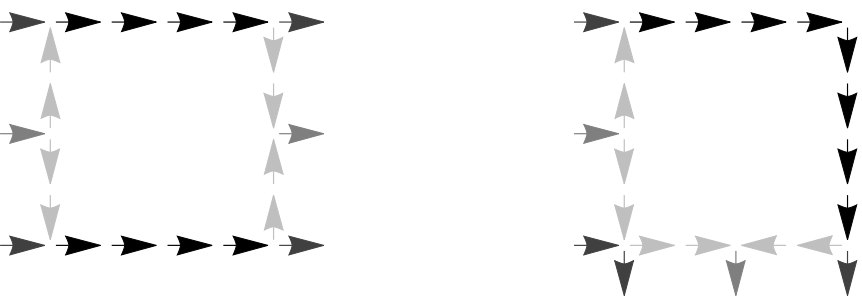

Figure 7.20. Replacement flows $\mathcal{J}_{1010}$ (left) and $\mathcal{J}_{1100}$ (right) for $m=1$. In $\mathcal{J}_{1010}$ : the incoming flow intensities are $1 / 2-\mu$ upper/lower and $2 \mu$ center; here $\mu \approx 0.1258652$.
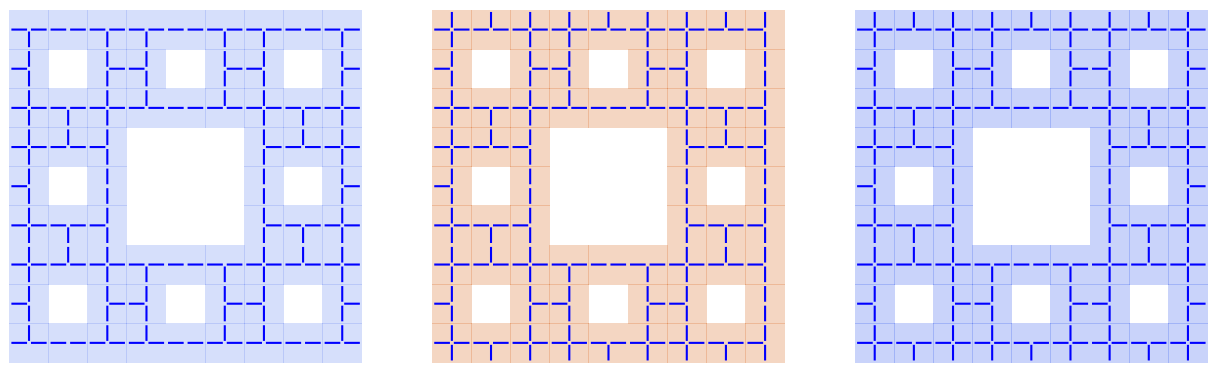

Figure 7.21. Three 2-supertiles for the alternative substitution.

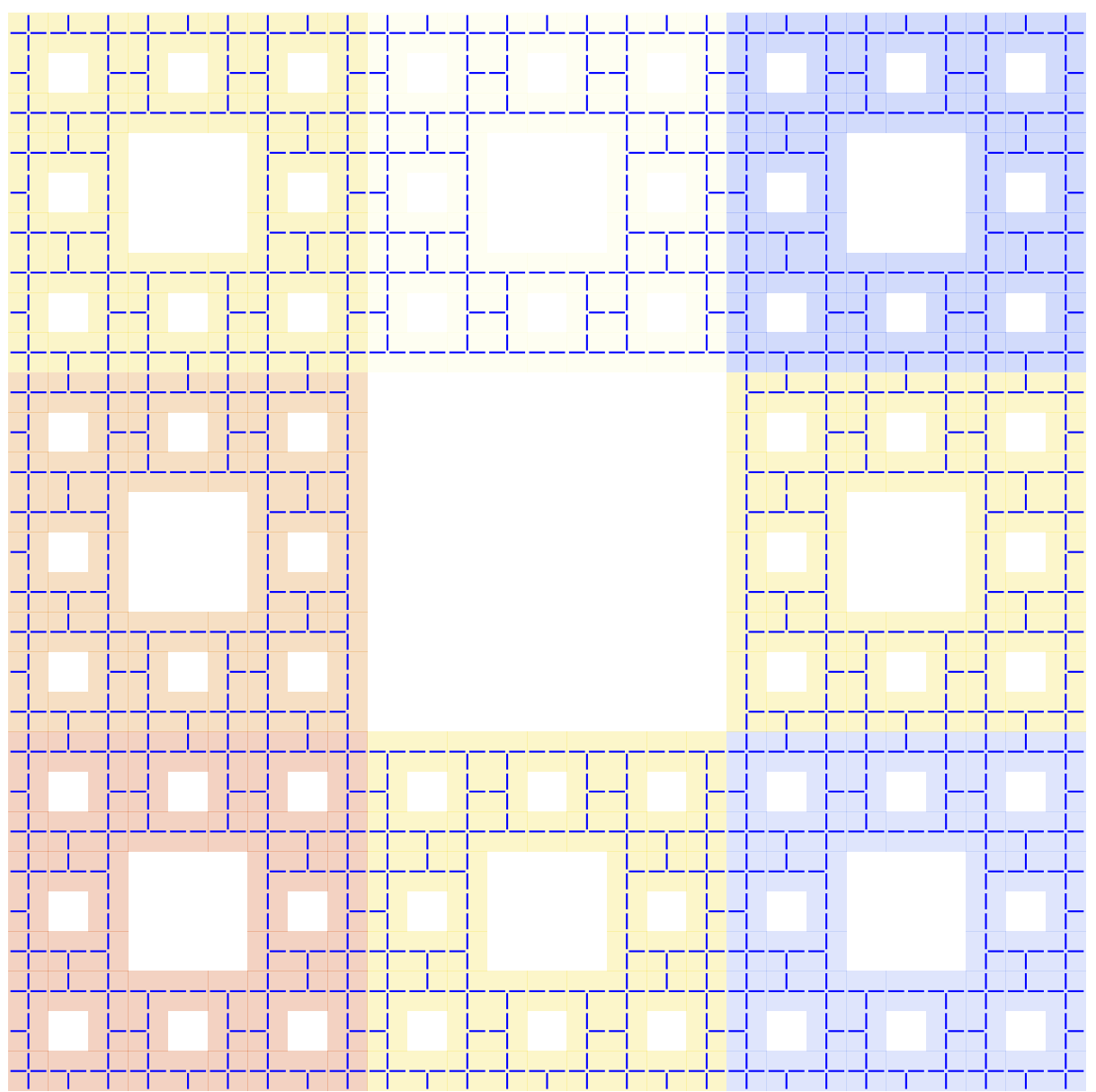

Figure 7.22. 3-supertile $\Phi^{3}\left(T_{C}\right)$ is a union of eight 2-supertiles. For left-to-right flows, its network is equivalent to $\tilde{\mathcal{G}}_{3}$ (carried by $\Phi^{3}\left(T_{A}\right)$ ) by removing the edges touching the top and bottom. 
We can repeat the formulas from Section 4 with $\mathrm{p}$ and $\mathrm{p}^{*}$ swapped (and some $*$ erased). In particular, the power ratio upon replacing $\left.\mathcal{J}\right|_{T}$ with flux vector $\mathbf{x}$ by the optimal combination of pure replacement flows is (cf. (4.10))

$$
\tilde{\rho}(\mathbf{x}):=\min \left\{\frac{P\left(\sum_{\mathcal{J}_{\circ} \in \mathfrak{J}} t\left(\mathcal{J}_{\circ}\right) \mathcal{J}_{\circ}\right)}{\sum_{i=1}^{4}\left|x_{i}\right|^{p^{*}}}: \sum_{\mathcal{J}_{\circ} \in \mathfrak{J}} t\left(\mathcal{J}_{\circ}\right) \mathbf{f}\left(\mathcal{J}_{\circ}\right)=\mathbf{x}, \mathbf{t}=\left(t\left(\mathcal{J}_{\circ}\right)\right) \in \mathbf{R}^{\mathfrak{J}}\right\} .
$$

Keep in mind that $\tilde{\rho}(\mathbf{x})$ depends on $m \in \mathbf{N}$, which we keep temporarily fixed. Proposition 5.1 has the following analogue:

Proposition 7.2. For $m \in \mathbf{N}, \operatorname{dim}_{A R}(X) \geq \mathrm{p}$ if

$$
\tilde{\rho}_{m}^{(\mathrm{rep})}(\mathrm{p}):=\max _{\mathbf{x} \in \mathbf{R}^{4}: \mathbf{x} \cdot \mathbf{1}=0} \tilde{\rho}(\mathbf{x})<1 .
$$

Proof. Summing over all tiles

$$
P\left(\mathcal{J}^{\prime}\right) \leq \tilde{\rho}_{m}^{(\mathrm{rep})}(\mathrm{p}) P(\mathcal{J})
$$

which, in terms of resistances, gives

$$
\tilde{R}_{n+m}(\mathrm{p}) \leq \tilde{\rho}_{m}^{(\mathrm{rep})}(\mathrm{p})^{\mathrm{p} / \mathrm{p}^{*}} \tilde{R}_{n}(\mathrm{p}) \quad(n \in \mathrm{N}) .
$$

If $\tilde{\rho}_{m}^{\text {(rep) }}(\mathrm{p})<1$, then $(7.5)$ guarantees $\lim _{n \rightarrow \infty} \tilde{R}_{n}(\mathrm{p})=0$ (via Corollary 1.4), and we are done by Theorem 1.2.

As before, in the game of minimizing the maximal (replacement) power ratio $\tilde{\rho}_{m}^{(\text {rep })}(\mathrm{p})$, one hopes that it is already attained as the pure power ratio (corresponding to $\mathbf{x}=(1,0,-1,0))$, defined by (cf. $(5.5))$ :

$$
\tilde{\rho}_{m}(\mathrm{p}):=\frac{\tilde{P}_{m}}{\tilde{P}_{0}}=\frac{\tilde{P}_{m}}{2}=\left(\frac{\tilde{R}_{m}(\mathrm{p})}{\tilde{R}_{0}(\mathrm{p})}\right)^{\mathrm{p}^{*} / \mathrm{p}}
$$

where we used that $\tilde{R}_{0}(\mathrm{p})=2^{\mathrm{p} / \mathrm{p}^{*}}$ (from two serial unit resistors) and $\tilde{P}_{0}=2$. However, the counterpart of Theorem 5.2 remains a conjecture.

Conjecture 7.3. For any $m \in \mathbf{N}$ and $\mathrm{p} \in(1,2)$, the maximal power ratio is attained on the pure replacement flows and (therefore) satisfies

$$
\tilde{\rho}_{m}^{(\mathrm{rep})}(\mathrm{p})=\tilde{\rho}_{m}(\mathrm{p}) \text {. }
$$

Nevertheless, we have the following weaker result.

Theorem 7.4. For any $m \in \mathbf{N}$ and $\mathrm{p} \in(1,2)$,

$$
\tilde{\rho}_{m}^{(\mathrm{rep})}(\mathrm{p}) \leq 2^{\frac{2-\mathrm{p}}{\mathrm{p}-1}} \tilde{\rho}_{m}(\mathrm{p}) .
$$

Proof. We repeat the proof of Theorem 5.2 with $\mathrm{p}$ and $\mathrm{p}^{*}$ swapped and one change in (5.14), substituting the following version of Clarkson's inequality valid for $\mathrm{p}^{*} \geq 2[14,28]:$

$$
\|\mathbf{x}+\mathbf{y}\|_{\mathrm{p}^{*}}^{\mathrm{p}^{*}}+\|\mathbf{x}-\mathbf{y}\|_{\mathrm{p}^{*}}^{\mathrm{p}^{*}} \leq 2^{\mathrm{p}^{*}-2}\left(2\|\mathbf{x}\|_{\mathrm{p}^{*}}^{\mathrm{p}^{*}}+2\|\mathbf{y}\|_{\mathrm{p}^{*}}^{\mathrm{p}^{*}}\right) \quad\left(\mathbf{x}, \mathbf{y} \in \mathbf{R}^{4}\right)
$$

The only difference is the extra multiplicative penalty factor $2^{\mathrm{p}^{*}-2}$. It remains to check that $\frac{2-\mathrm{p}}{\mathrm{p}-1}=-1+\frac{1}{\mathrm{p}-1}=-1+\frac{\mathrm{p}^{*}}{\mathrm{p}}=-1+\left(1-\frac{1}{\mathrm{p}^{*}}\right) \mathrm{p}^{*}=\mathrm{p}^{*}-2$.

Digressing, we record the following counterpart of Corollary 5.3:

Corollary 7.5. For $\mathrm{p} \in(1,2)$,

$$
\tilde{R}_{n+m} \leq 2^{3-2 \mathrm{p}} \tilde{R}_{n}(\mathrm{p}) \tilde{R}_{m}(\mathrm{p}) \quad(m, n \in \mathbf{N}) .
$$


Proof. In view of Theorem 7.4 , equation $(7.6)$, and $\tilde{R}_{0}(\mathrm{p})=2^{\mathrm{p} / \mathrm{p}^{*}}=2^{\mathrm{p}-1}$, inequality (7.5) gives:

$$
\tilde{R}_{n+m}(\mathrm{p}) \leq\left(2^{\frac{2-\mathrm{p}}{\mathrm{p}-1}} \tilde{\rho}_{m}(\mathrm{p})\right)^{\mathrm{p} / \mathrm{p}^{*}} \tilde{R}_{n}(\mathrm{p})=2^{\frac{2-\mathrm{p}}{\mathrm{p}-1} \frac{\mathrm{p}}{\mathrm{p}^{*}}} \frac{\tilde{R}_{m}(\mathrm{p})}{\tilde{R}_{0}(\mathrm{p})} \tilde{R}_{n}(\mathrm{p})=2^{2-\mathrm{p}} 2^{1-\mathrm{p}} \tilde{R}_{m}(\mathrm{p}) \tilde{R}_{n}(\mathrm{p})
$$

Going back to pursuing lower bounds, Theorem 7.4 and Proposition 7.2 give:

Corollary 7.6. For $m \in \mathbf{N}, \operatorname{dim}_{A R}(X) \geq \mathrm{p}$ if

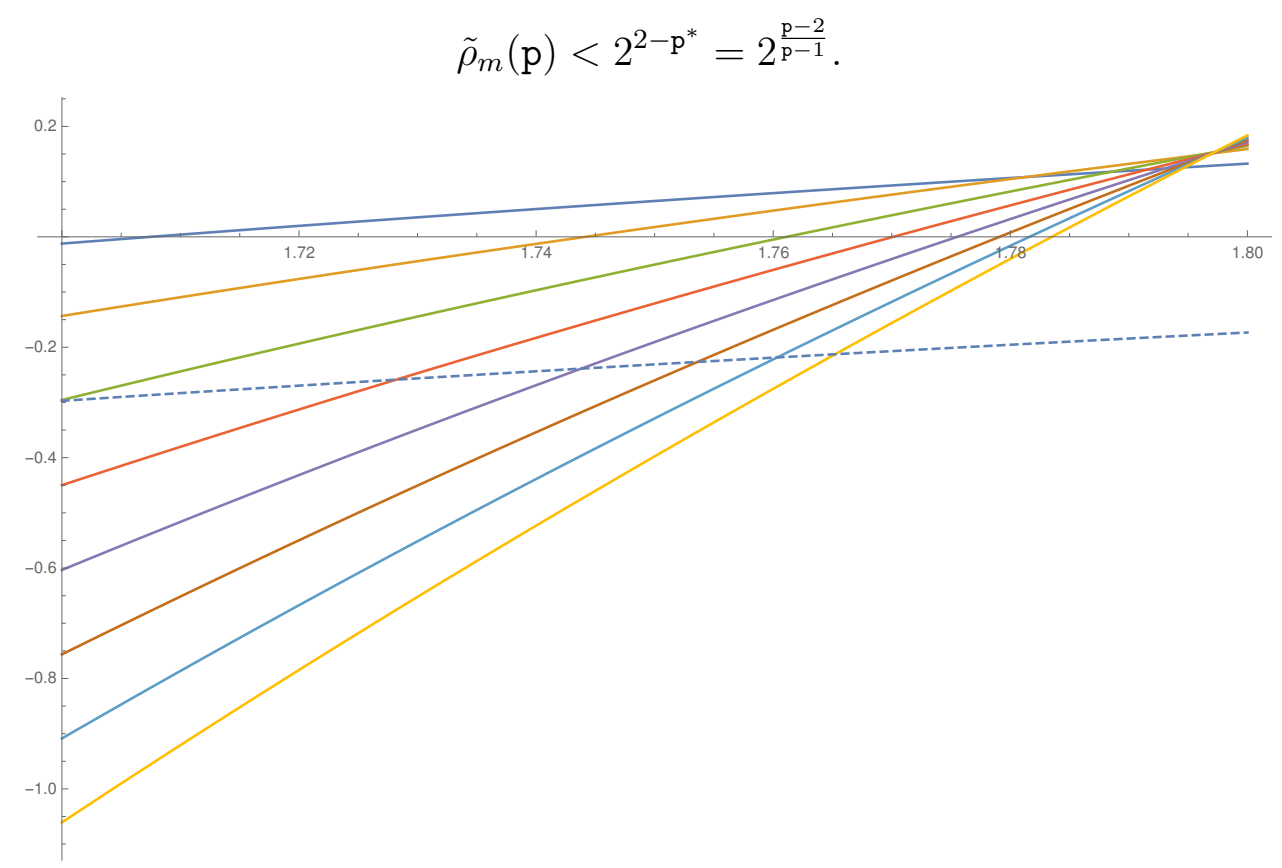

Figure 7.23. $\log$ of the power ratio $\ln \tilde{\rho}_{m}(\mathrm{p})$ for $\tilde{\mathcal{G}}_{m}$ as a function of $\mathrm{p}$, steepening with increasing $m$ (for $m=1, \ldots, 8)$. The dashed reference graph is that of $\frac{\mathrm{p}-2}{\mathrm{p}-1} \ln 2$.

By computing the plots of $\ln \tilde{\rho}_{m}(\mathrm{p})$ (see Figure 7.23 ) for increasing values of $m$ and intersecting with the reference plot of $\frac{\mathrm{p}-2}{\mathrm{p}-1} \ln 2$ (to $10^{-8}$ precision, see Section 8 ), we find $\mathrm{p}$ for which $\tilde{\rho}_{m}(\mathrm{p})<2^{\frac{\mathrm{p}-2}{\mathrm{p}-1}}$ is nearly an equality, as listed below.

Proposition 7.7. (Rigorous computer assisted bounds)

$$
\begin{array}{ll}
\operatorname{dim}_{A R}(X) \geq 1.147018 & (m=1), \\
\operatorname{dim}_{A R}(X) \geq 1.631569 & (m=2), \\
\operatorname{dim}_{A R}(X) \geq 1.699604 & (m=3), \\
\operatorname{dim}_{A R}(X) \geq 1.728198 & (m=4), \\
\operatorname{dim}_{A R}(X) \geq 1.743808 & (m=5), \\
\operatorname{dim}_{A R}(X) \geq 1.753609 & (m=6), \\
\operatorname{dim}_{A R}(X) \geq 1.760330 & (m=7), \\
\operatorname{dim}_{A R}(X) \geq 1.765225 & (m=8) .
\end{array}
$$

Proof (computer assisted). Fix one of the listed $\mathrm{p}$ (and the corresponding $m$ ). By Corollary 7.6, one just has to show $\tilde{\rho}_{m}(\mathrm{p})<2^{\frac{\mathrm{p}-2}{\mathrm{p}-1}}$. To do this we computed a flow $\mathcal{J}_{1010}^{\text {approx }}$ that is an approximation to the pure replacement current $\mathcal{J}_{1010}$. Note that 
$\tilde{\rho}_{m}(\mathrm{p}) \leq \tilde{P}_{\text {approx }} / 2$ where $\tilde{P}_{\text {approx }}:=P\left(\mathcal{J}_{1010}^{\text {approx }}\right)$, which we evaluated with sufficient numerical precision to guarantee $\tilde{P}_{\text {approx }} / 2<2^{\frac{\mathrm{p}-2}{\mathrm{p}-1}}$.

Taking Conjecture 7.3 for granted, much better bounds are found by selecting $\mathrm{p}$ that are just below the zeros of $\ln \tilde{\rho}_{m}(\mathrm{p})$.

Proposition 7.8. (Computer assisted bounds, assuming Conjecture 7.3)

$$
\begin{array}{ll}
\operatorname{dim}_{A R}(X) \geq 1.707491 & (m=1 *), \\
\operatorname{dim}_{A R}(X) \geq 1.744155 & (m=2 *), \\
\operatorname{dim}_{A R}(X) \geq 1.761130 & (m=3 *), \\
\operatorname{dim}_{A R}(X) \geq 1.770127 & (m=4 *), \\
\operatorname{dim}_{A R}(X) \geq 1.775526 & (m=5), \\
\operatorname{dim}_{A R}(X) \geq 1.779092 & (m=6), \\
\operatorname{dim}_{A R}(X) \geq 1.781618 & (m=7), \\
\operatorname{dim}_{A R}(X) \geq 1.783499 & (m=8) .
\end{array}
$$

The stars by $m=1, \ldots, 4$ indicate that we actually verified Conjecture $7.3 \mathrm{nu}-$ merically for those values of $m$ (and the corresponding $\mathrm{p}$ ). The explanation of how this was done follows.

(Numerical) Verification of Conjecture 7.3. In absence of a conceptual proof of the conjecture, we resort to its verification for concrete $m$ and $\mathrm{p}$ by explicit study of the function $\tilde{\rho}(\mathbf{x})$ maximized in (7.3). Because $\tilde{\rho}(\mathbf{x})$ is invariant under scaling we can normalize the absolute flux $\frac{1}{2} \sum_{i=1}^{4}\left|x_{i}\right|$ and solve the maximization (7.2) over the two dimensional domain

$$
\Sigma:=\left\{\mathbf{x} \in \mathbf{R}^{4}: \mathbf{x} \cdot \mathbf{1}=0, \frac{1}{2} \sum_{i=1}^{4}\left|x_{i}\right|=1\right\} .
$$

$\Sigma$ is the boundary of a convex 3 dimensional polyhedron. By rotating, it suffices to consider $\mathbf{x} \in \Sigma$ with $\left|x_{1}\right|=\max _{i=1}^{4}\left\{\left|x_{i}\right|\right\}$. Finally, using the reflection symmetry and flow reversal reduces considerations to the following subdomains of $\Sigma$ :

- three-way split flow (one in and three out):

$$
\Sigma_{4 s s s}:=\left\{\mathbf{x} \in \Sigma: x_{1} \geq 0 \geq x_{2}, x_{3}, x_{4} \text { and }\left|x_{1}\right| \geq\left|x_{2}\right|,\left|x_{3}\right|,\left|x_{4}\right|\right\} .
$$

- saddle flow (two opposite, in and out):

$\Sigma_{4312}:=\left\{\mathbf{x} \in \Sigma: x_{1}, x_{3} \geq 0 \geq x_{2}, x_{4}\right.$ and $\left|x_{1}\right| \geq\left|x_{3}\right|,\left|x_{2}\right|$ and $\left.\left|x_{2}\right| \geq\left|x_{4}\right|\right\}$.

- turning cross flow (strongest turns):

$$
\Sigma_{4123}:=\left\{\mathbf{x} \in \Sigma: x_{1}, x_{2} \geq 0 \geq x_{3}, x_{4} \text { and }\left|x_{1}\right| \geq\left|x_{4}\right| \geq\left|x_{3}\right| \geq\left|x_{2}\right|\right\} .
$$

- straight cross flow (strongest goes straight):

$$
\Sigma_{4132}:=\left\{\mathbf{x} \in \Sigma: x_{1}, x_{2} \geq 0 \geq x_{3}, x_{4} \text { and }\left|x_{1}\right| \geq\left|x_{3}\right| \geq\left|x_{4}\right| \geq\left|x_{2}\right|\right\} .
$$

The labeling of domains is just a convenient recording of the absolute strengths of the flow rates $x_{i}$, e.g., $\Sigma_{4132}$ indicates that $\left|x_{1}\right|$ is largest followed by $\left|x_{3}\right|$ and then $\left|x_{4}\right|$, with $\left|x_{2}\right|$ being the smallest. $\Sigma_{4 s s s}$ signifies that $\left|x_{1}\right|$ is largest with $\left|x_{2}\right|,\left|x_{3}\right|,\left|x_{4}\right|$ smaller, in no particular order.

For any fixed $\mathbf{x}$, to obtain an upper bound on $\tilde{\rho}(\mathbf{x})$, we make an explicit choice of the mixing parameters $\mathbf{t}=\left(t\left(\mathcal{J}_{\circ}\right)\right)_{\mathcal{J}_{\circ} \in \mathfrak{J}}$ used to generate the replacement flow 
$\mathcal{J}^{\prime}:=\sum_{\mathcal{J}_{\circ} \in \mathfrak{J}} t\left(\mathcal{J}_{\circ}\right) \mathcal{J}_{\circ}$ in the convex minimization (7.2) defining $\tilde{\rho}(\mathbf{x})$. Our choices are as follows (where we describe each $\mathbf{x}$ by two real parameters $s$ and $t$ ).

$$
\begin{aligned}
\Sigma_{4 s s s} & =\{\mathbf{x}=(1,-t,-s, s+t-1): s, t \geq 0, s+t \leq 1\}, \\
\mathcal{J}^{\prime}(s, t) & :=s \mathcal{J}_{1010}+t \mathcal{J}_{1100}+(1-s-t) \mathcal{J}_{1001}, \\
\Sigma_{4312} & =\{\mathbf{x}=(s,-t, 1-s, t-1): 1 / 2 \leq t \leq s \leq 1\}, \\
\mathcal{J}^{\prime}(s, t) & :=s \mathcal{J}_{1100}+(1-s) \mathcal{J}_{0011}+(s-t) \mathcal{J}_{0101}, \\
\Sigma_{4123} & =\{\mathbf{x}=(1-s, s,-t, t-1): 0 \leq s \leq t \leq 1 / 2\}, \\
\mathcal{J}^{\prime}(s, t) & :=s \mathcal{J}_{0101}+t \mathcal{J}_{1010}+(1-s-t) \mathcal{J}_{1001} \\
\Sigma_{4132} & =\{\mathbf{x}=(1-s, s,-t, t-1): t \geq 1 / 2, s+t \leq 1\}, \\
\mathcal{J}^{\prime}(s, t) & :=s \mathcal{J}_{0101}+t \mathcal{J}_{1010}+(1-s-t) \mathcal{J}_{1001} .
\end{aligned}
$$

By this construction

$$
\tilde{\rho}(\mathbf{x}) \leq \tilde{\rho}^{+}(\mathbf{x}):=\frac{P\left(\mathcal{J}^{\prime}\right)}{\sum_{i=1}^{4}\left|x_{i}\right|^{\mathrm{p}^{*}}}
$$

where $\mathcal{J}^{\prime}$ and thus also $\tilde{\rho}^{+}(\mathbf{x})$ depend only on $(s, t)$. To verify the conjecture, we check that $\tilde{\rho}^{+}(\mathbf{x})$, as a function of $(s, t)$, attains its maximum at the corners of the triangular domains $\Sigma_{4 s s s}, \ldots, \Sigma_{4132}$. We then evaluate it there to see that the maximum is $\tilde{\rho}^{+}((1,0,-1,0))=\tilde{\rho}_{m}(\mathrm{p})$. This is convincingly done by using a computer to generate contour plots of $\rho^{+}(\mathbf{x})$ over the four domains and witnessing their convexity. We performed this computation for $m=1, \ldots, 4$. For example, Figure 7.25 depicts plots of $\tilde{\rho}^{+}(\mathbf{x})$ for $m=1$ and $\mathrm{p}=1.707491$. (We further comment on this case below.)

Remark 7.9. That the plots of the power ratios (Figure 7.25) over $\Sigma_{4123}$ and $\Sigma_{4132}$ coincide upon mapping $(s, t)$ to $(s, 1-t)$ is explained by interchanging $x_{3}$ and $x_{4}$. Indeed, the flow $\mathcal{J}_{4123}^{\prime}=s \mathcal{J}_{0101}+t \mathcal{J}_{1010}+(1-s-t) \mathcal{J}_{1001}$ transforms then into

$$
\begin{aligned}
s \mathcal{J}_{0110}+t \mathcal{J}_{1001}+(1-s-t) \mathcal{J}_{1010} & =\left(s \mathcal{J}_{0101}+s \mathcal{J}_{1010}+(t-s) \mathcal{J}_{1001}\right)+(1-s-t) \mathcal{J}_{1010} \\
& =s \mathcal{J}_{0101}+(1-t) \mathcal{J}_{1010}+(t-s) \mathcal{J}_{1001}
\end{aligned}
$$

which is the $\mathcal{J}_{4132}^{\prime}$ flow with parameters $(s, 1-t)$.

To conclude, we attend to the lower bound in Theorem 1.1. Note that it comes from rounding down to four digits the bound given by Proposition 7.8 for $m=1$. This is the simplest instance of our method, dealing with just a few resistors, making it easier to detail all that goes into the proof and clearly articulate the level of computer assistance.

Proof of the lower bound in Theorem 1.1: We set $\mathrm{p}=1.707491$. First, we find a good unit flux flow $\mathcal{J}_{1010}^{\text {approx }}$ in $\tilde{\mathcal{G}}_{1}$. Because of symmetry, we look among the flows as depicted in Figure 7.20, which can be parametrized by just one real parameter $\mu \in[0,1 / 2]$. This parameter can be selected ad hoc to be $\mu=0.1258652$. This selection happens to be an approximate minimizer of the p-power, with $\mathrm{p}=1.707491$, dissipated in the 22 unit resistors with non-zero current. By joining and using symmetry, the number of resistors can be reduced to 4 - which yields the four terms in the formula (7.11), below. Denote the power of $\mathcal{J}_{1010}^{\text {approx }}$ by $\tilde{P}_{1}^{\prime}$. By construction $\tilde{P}_{1} \leq \tilde{P}_{1}^{\prime}$.

A small explicit computation (for $\mathrm{p}=1.707491$ and $\mu=0.1258652$ ) gives

$$
\tilde{\rho}_{1}=\frac{\tilde{P}_{1}}{2} \leq \frac{\tilde{P}_{1}^{\prime}}{2}=4|1 / 2|^{\mathrm{p}^{*}}+4|\mu|^{\mathrm{p}^{*}}+2|1 / 2-\mu|^{\mathrm{p}^{*}}+|2 \mu|^{\mathrm{p}^{*}} \approx 0.9999996<1 .
$$


It remains to verify Conjecture 7.3 , which can be done by showing that $\tilde{\rho}^{+}(\mathbf{x}) \leq$ $\frac{\tilde{P}_{1}^{\prime}}{2}$ for all $\mathbf{x}$. As already mentioned, it suffices to check that $\tilde{\rho}^{+}(\mathbf{x})$ - a concrete function of the parameters $s$ and $t$, evaluated by using $\mathcal{J}_{1010}^{\text {approx }}$-attains its maximum at the corners of the domains. We shy away from solving this calculus exercise here and only present computer generated contour plots (Figure 7.25), where the sought behavior of $\tilde{\rho}^{+}(\mathbf{x})$ is apparent.

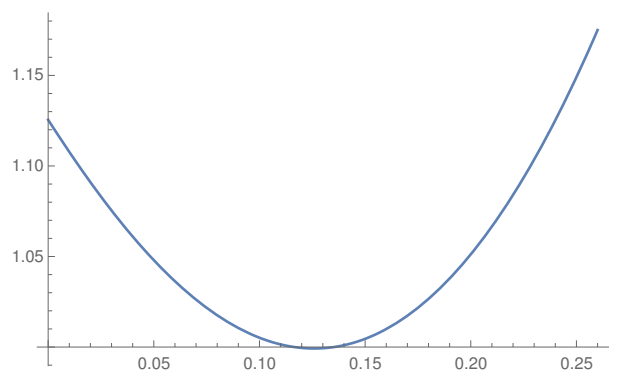

Figure 7.24. Power of replacement flow $\mathcal{J}_{1010}$ (depicted in Figure 7.20) is a convex function of $\mu$ with a unique minimum near $\mu=0.1258652$, where the power ratio is $0.9999996<1$ for $p=1.707491$.
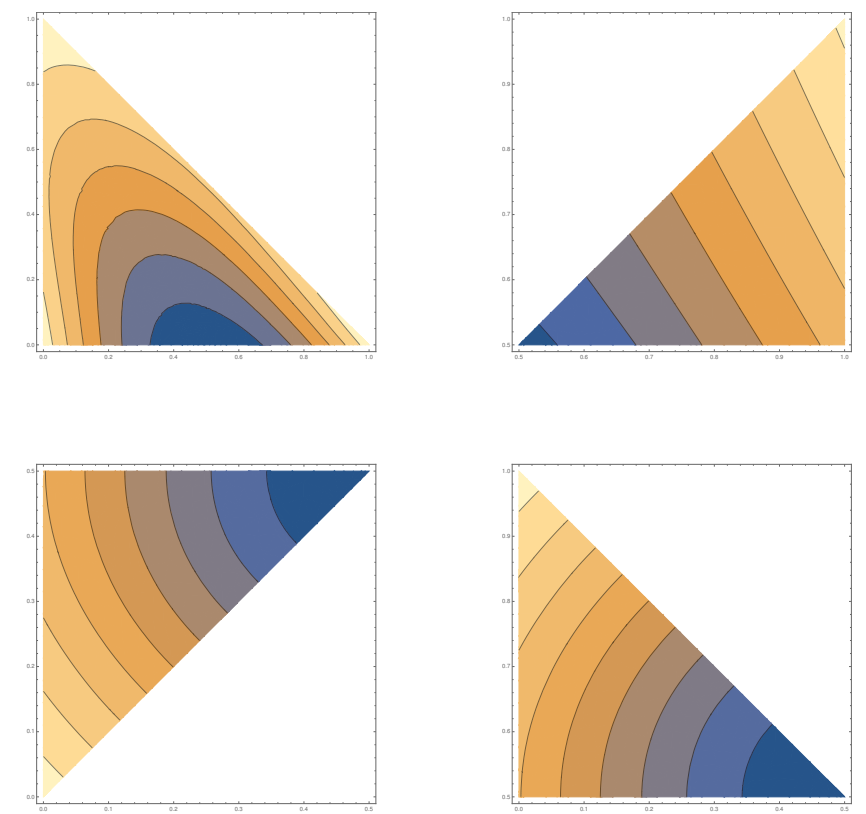

Figure 7.25. The power ratio of the replacement flow $\mathcal{J}^{\prime}$ as a function of parameters $s$ (horizontal axis) and $t$ (vertical axis). $\mathcal{J}_{4 s s s}, \mathcal{J}_{4312}$ (above) and $\mathcal{J}_{4123}, \mathcal{J}_{4132}$ (below). Lighter color/shading indicates higher power ratio. We use $\mathrm{p}=1.707491$ - which is nearly optimal for $m=1$ - and an approximation $\mathcal{J}_{1010}^{\text {approx }}$. $\left(\mathcal{J}_{4123}\right.$ and $\mathcal{J}_{4132}$ are congruent by Remark 7.9.)

\section{Numerics}

The numerics behind the prediction $\operatorname{dim}_{A R}(X) \approx 1.7965$ and our upper bounds for $\operatorname{dim}_{A R}(X)$ are based on the computation of the p-resistance $R_{n}(\mathrm{p})$ and the corresponding currents and potentials for the standard networks $\mathcal{G}_{n}$ with $n=1, \ldots, 8$. (The resistance, current, and potential for the dual networks $\mathcal{G}_{n}^{*}$ are then computed by the duality relations.) Below we provide some details about the numerics on $\mathcal{G}_{n}$. The lower bounds rely on the analogous computations for the alternative networks $\tilde{\mathcal{G}}_{n}$. 
We solve (1.3) by using the primal-dual approach, i.e., simultaneous solving of the Euler-Lagrange equations (1.10) for potentials $\mathcal{U}(v)$ and currents $\mathcal{J}(e)$, subject to Kirchhoff's first law at the interior vertices and the flux/potential drop boundary conditions (1.11). By using symmetry, we confine the computation to the bottom left quarter of the network $\mathcal{G}_{n}$.

To solve the nonlinear equations (1.10), we use Mathematica's FindRoot function, which implements a variant of quasi-Newton method called Broyden-FletcherGoldfarb-Shanno algorithm (in its limited-memory version (L-BFGS) for systems of size $>250)$. We run the code on a single core Intel(R) Xeon(R) CPU E5-2640 v3 $2.60 \mathrm{GH}$ with $188 \mathrm{~GB}$ of RAM, under Ubuntu 14.04 OS. (Incidentally, for $n=6$, an eight years old laptop suffices and only about 1.3 GB of RAM is used in sub 30 min time.)

Computing the solutions to 8-digit precision for $n<8$ and 7 digit precision for $n=8$ takes no more than 8 quasi-Newton steps. When $n=8$, the quarter-network has 10,065, 674 edges and 5,275,720 vertices, and Mathematica utilizes virtually all of the available RAM memory, taking circa 54 hours to finish. Table 8.1 reports the values of the pure power ratio $\rho_{n}(\mathrm{p})=\frac{P_{n}}{P_{0}}=\left(\frac{R_{n}(\mathrm{p})}{R_{0}(\mathrm{p})}\right)^{\mathrm{p}^{*} / \mathrm{p}}$ for several $\mathrm{p}$ and $n$, together with the computation time. (Recall that $R_{0}=\frac{1}{2}$, and $P_{0}=2$ because we passed unit current through each of the two resistors in $\mathcal{G}_{0}$, so the total flux $J_{0}=1+1=2$.)

\begin{tabular}{|l|l|l|l|l|l|l|l|c|}
\hline$n \quad p$ & 1.797 & 1.798 & 1.799 & 1.800 & 1.801 & 1.802 & 1.803 & seconds \\
\hline 1 & 0.93302724 & 0.93438418 & 0.93573978 & 0.93709402 & 0.93844692 & 0.93979847 & 0.94114868 & $2.4 \cdot 10^{-2}$ \\
\hline 2 & 0.91092471 & 0.91356353 & 0.91620350 & 0.91884459 & 0.92148680 & 0.92413012 & 0.92677453 & $9.6 \cdot 10^{-2}$ \\
\hline 3 & 0.90361233 & 0.90751319 & 0.91142121 & 0.91533640 & 0.91925872 & 0.92318816 & 0.92712471 & $4.7 \cdot 10^{-1}$ \\
\hline 4 & 0.90042774 & 0.90558235 & 0.91075365 & 0.91594164 & 0.92114631 & 0.92636766 & 0.93160569 & $3.6 \cdot 10^{0}$ \\
\hline 5 & 0.89880763 & 0.90521203 & 0.91164610 & 0.91810988 & 0.92460341 & 0.93112675 & 0.93767996 & $5.4 \cdot 10^{1}$ \\
\hline 6 & 0.89799388 & 0.90564725 & 0.91334673 & 0.92109246 & 0.92888457 & 0.93672321 & 0.94460851 & $4.5 \cdot 10^{2}$ \\
\hline 7 & 0.89765931 & 0.90656283 & 0.91553238 & 0.92456824 & 0.93367071 & 0.94284010 & 0.95207669 & $5.4 \cdot 10^{3}$ \\
\hline 8 & 0.89763003 & 0.90778602 & 0.91803144 & 0.92836683 & 0.93879270 & 0.94930960 & 0.95991807 & $9.4 \cdot 10^{4}$ \\
\hline
\end{tabular}

Figure 8.1. Power ratios $\rho_{n}(\mathrm{p})$ and their computing times.

Between the 0.001 spaced values of $\mathrm{p}$ (in Table 8.1), we interpolated $\rho_{n}(\mathrm{p})$ by cubic splines (via Interpolation [] function in Mathematica), with the interpolation error estimated by using the standard centered differences for the fourth derivative, [15]:

$$
\text { interpolation error } \leq \frac{5}{384} 0.001^{4} \max \left|\rho_{n}^{(4)}(\mathrm{p})\right| \leq 1.5 \cdot 10^{-11},
$$

well below the $10^{-8}$ precision threshold.

The interpolated plots of $\ln \rho_{n}(\mathrm{p})$ are depicted in Figure 8.26. The inset picture depicts the plots of detrended functions $g_{n}(\mathrm{p}):=\frac{\ln \rho_{n}(\mathrm{p})-\ln \rho_{1}(\mathrm{p})}{n-1}$ for $n=2, \ldots, 8$, which are coarsely approximated by $a_{n} \mathrm{p}+b_{n}$ with the slopes $a_{n} \approx 1.4$, seemingly (yet imperceptibly in the figure) decreasing to $a_{\infty} \approx 1.38$, and with $b_{n}$ tending to some value $b_{\infty} \simeq-2.52$. That is, we have an approximate functional form $\ln \rho_{n}(\mathrm{p}) \approx$ $n\left(a_{\infty} \mathrm{p}+b_{\infty}\right)+a_{0} \mathrm{p}+b_{0}$, describing a family of steepening lines pivoting about a single point, whose abscissa $\mathrm{p}_{\infty}:=-b_{\infty} / a_{\infty}$ is a rough approximation to $\mathrm{p}_{\text {res }}$ (cf. Figure 1.4). To better resolve $\mathrm{p}_{\text {res }}$, we study the abscissas $\mathrm{p}_{i, j}$ of the intersection points between the $i$-th and $j$-th graph, which are listed in Table 8.2.

We extrapolate the sequence of points $\left(\mathrm{p}_{n}, y_{n}\right)$ where the graphs of $\ln \rho_{n}(\mathrm{p})$ and $\ln \rho_{n-1}(\mathrm{p})$ intersect $(n=2, \ldots, 8)$. (The $\mathrm{p}_{n}=\mathrm{p}_{n, n-1}$ appear on the diagonal of 
Table 8.2.) Looking at Figure 8.26, these points appear to move left-and-down along the envelope of the steepening graphs and conjecturally converge to a single point ( $\left.\mathrm{p}_{\text {res }}, y_{\mathrm{res}}\right)$, where the abscissa $\mathrm{p}_{\text {res }}$ is the conformal dimension and the ordinate $y_{\text {res }}$ gives the critical resistance; specifically, $y_{\text {res }}=\frac{\mathrm{p}^{*}}{\mathrm{p}} \ln \frac{R\left(\mathrm{p}_{\mathrm{res}}\right)}{\frac{1}{2}}$ where $R\left(\mathrm{p}_{\mathrm{res}}\right)$ is as in Conjecture 1.5.

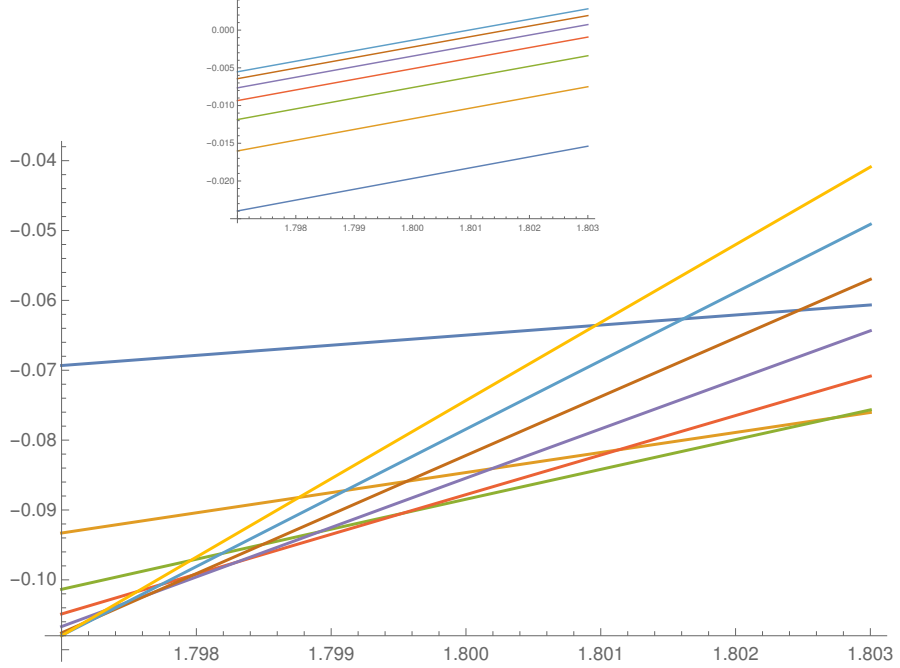

Figure 8.26. $\log$ of the power ratio $\log \rho_{n}(\mathrm{p})$ for $1.797 \leq \mathrm{p} \leq 1.803$ (for $n=1, \ldots, 8$ ); cf. Figure 1.4. Inset: graphs of detrended functions $g_{n}(\mathrm{p}):=\frac{\ln \rho_{n}(\mathrm{p})-\ln \rho_{1}(\mathrm{p})}{n-1}$.

\begin{tabular}{|l|l|l|l|l|l|l|l|}
\hline j & 2 & 3 & 4 & 5 & 6 & 7 & 8 \\
\hline 1 & 1.81398 & 1.80837 & 1.80544 & 1.80366 & 1.80247 & 1.80161 & 1.80096 \\
\hline 2 & & 1.80273 & 1.80113 & 1.80019 & 1.79956 & 1.79911 & 1.79876 \\
\hline 3 & & & 1.79953 & 1.79891 & 1.79849 & 1.79819 & 1.79796 \\
\hline 4 & & & & 1.79829 & 1.79797 & 1.79774 & 1.79756 \\
\hline 5 & & & & & 1.79765 & 1.79746 & 1.79732 \\
\hline 6 & & & & & & 1.79727 & 1.79715 \\
\hline 7 & & & & & & & 1.79702 \\
\hline
\end{tabular}

Table 8.2. Values $\mathrm{p}_{i, j}$ of $\mathrm{p}$ where $\rho_{i}(\mathrm{p})=\rho_{j}(\mathrm{p})$.

To extrapolate the value $\mathrm{p}_{\text {res }}$ from mere first seven terms of the sequence $\left(\mathrm{p}_{n}\right)_{n=2}^{\infty}$ we utilize Shanks transform, as done in [4]. The difference quotients

$$
\left(\frac{\mathrm{p}_{n+1}-\mathrm{p}_{n}}{\mathrm{p}_{n}-\mathrm{p}_{n-1}}\right)_{n=3}^{7} \approx(0.2847,0.3842,0.5209,0.5985,0.6398)
$$

suggest that $\mathrm{p}_{n}$ converge geometrically (with the asymptotic quotient $\leq 0.7$ ). This justifies acceleration of this convergence via Shanks transform, whereby $\left(\mathrm{p}_{n}\right)_{n=2}^{8}$ yields points

$$
\hat{\mathrm{p}}_{n}=\mathrm{p}_{n+1}-\frac{\mathrm{p}_{n+1}-\mathrm{p}_{n}}{\mathrm{p}_{n+1}-2 \mathrm{p}_{n}+\mathrm{p}_{n-1}}\left(\mathrm{p}_{n+1}-\mathrm{p}_{n}\right) \quad(n=3, \ldots, 7),
$$

reported to five digits past the decimal point as

$$
\left(\hat{\mathrm{p}}_{n}\right)_{n=3}^{7}=(1.79825,1.79753,1.79696,1.79670,1.79659) .
$$

The associated difference quotients

$$
\left(\frac{\hat{\mathrm{p}}_{n+1}-\hat{\mathrm{p}}_{n}}{\hat{\mathrm{p}}_{n}-\hat{\mathrm{p}}_{n-1}}\right)_{n=4}^{6}=(0.787742,0.453624,0.423594)
$$


again indicate geometric convergence, prompting a repeated Shanks transform yielding $^{3}$

$$
\left(\hat{\hat{\mathrm{p}}}_{n}\right)_{n=4}^{6}=(1.79484,1.79648,1.79651) .
$$

On the basis of the last value we predict the five significant digits of the conformal dimension to be

$$
\mathrm{p}_{\mathrm{res}} \approx 1.7965 \text {. }
$$

We checked that this prediction is not degraded by amplification of the round-off error under the Shanks transform. Indeed, the precision of $\mathrm{p}_{n}$ was $10^{-8}$ and the ratio of the first and second differences in (8.1) does not exceed 3 , so $\hat{\mathrm{p}}_{n}$ retain at least $10^{-7}$ precision. The situation is similar for the second application of Shanks transform, so we have confidence that any errors in the five reported digits $\hat{\hat{p}}_{n}$ are inherent to the departure of the sequence $\left(\mathrm{p}_{n}\right)$ from geometric behavior.

\section{Appendix: Proof of Lemma 7.1}

We start with a simple general criterion allowing rough comparison of extremal lengths. Let $\mathcal{G}=(\mathcal{V}, \mathcal{E})$ and $\tilde{\mathcal{G}}=(\tilde{\mathcal{V}}, \tilde{\mathcal{E}})$ be two networks and $\Gamma$ and $\tilde{\Gamma}$ be two families of (vertices of) paths in $\mathcal{G}$ and $\tilde{\mathcal{G}}$, respectively. Given $M>0$, we shall say that $(\tilde{\mathcal{G}}, \tilde{\Gamma})$ is $M$-quasi-contained in $(\mathcal{G}, \Gamma)$ iff to each $\tilde{v} \in \tilde{\mathcal{V}}$ there is associated a subset $B(\tilde{v}) \subset \mathcal{V}$ - of which one should think as neighbors of $\tilde{v}$-such that:

(i) given any $\tilde{v}_{0}, \ldots, \tilde{v}_{k} \in \tilde{\mathcal{V}}$ that are vertices of a path $\tilde{\gamma} \in \tilde{\Gamma}$ there are $v_{0}, \ldots, v_{k^{\prime}} \in$ $\bigcup_{j=1}^{k} B\left(\tilde{v}_{j}\right)$ that are vertices of a path $\gamma \in \Gamma$;

(ii) for any $\tilde{v} \in \tilde{\mathcal{V}}$, the cardinality of $B(\tilde{v})$ does not exceed $M$;

(iii) for any $v \in \mathcal{V}$, the number of $\tilde{v} \in \mathcal{V}$ with $v \in B(\tilde{v})$ does not exceed $M$.

If $M$-quasi-containment holds both ways, we call $(\tilde{\mathcal{G}}, \tilde{\Gamma})$ and $(\mathcal{G}, \Gamma) M$-quasi-equal.

Fact 9.1. If $(\tilde{\mathcal{G}}, \tilde{\Gamma})$ is $M$-quasi-contained in $(\mathcal{G}, \Gamma)$ then the p-extremal lengths of $\Gamma$ and $\tilde{\Gamma}$ (defined as in (1.6) or (1.8)) satisfy

$$
\Lambda(\mathrm{p}) \leq M^{\mathrm{p}} \tilde{\Lambda}(\mathrm{p}) \quad(\mathrm{p}>1) .
$$

Proof. Given an admissible weight $\mathrm{m}: \mathcal{V} \rightarrow[0, \infty)$, we define $\tilde{\mathrm{m}}: \tilde{\mathcal{V}} \rightarrow[0, \infty)$ by

$$
\tilde{\mathrm{m}}(\tilde{v}):=\sum_{v \in B(\tilde{v})} \mathrm{m}(v)
$$

The weight $\tilde{\mathrm{m}}$ is admissible by construction because, for any $\tilde{\gamma} \in \tilde{\Gamma}$, taking $\gamma \in \Gamma$ as in (i) yields

$$
\sum_{\tilde{v} \in \tilde{\gamma}} \tilde{\mathrm{m}}(\tilde{v}) \geq \sum_{v \in \gamma} \mathrm{m}(v) \geq 1
$$

At the same time convexity of $t \mapsto t^{\mathrm{p}}$ and (ii) and (iii) give

$$
\sum_{\tilde{v} \in \tilde{\mathcal{V}}} \tilde{\mathrm{m}}(\tilde{v})^{\mathrm{p}}=\sum_{\tilde{v} \in \tilde{\mathcal{V}}}\left(\sum_{v \in B(\tilde{v})} \mathrm{m}(v)\right)^{\mathrm{p}} \leq \sum_{\tilde{v} \in \tilde{\mathcal{V}}} \sum_{v \in B(\tilde{v})} M^{\mathrm{p}-1} \mathrm{~m}(v)^{\mathrm{p}} \leq M^{\mathrm{p}-1} \sum_{v \in \mathcal{V}} M \mathrm{~m}(v)^{\mathrm{p}} .
$$

By arbitrariness of $\mathrm{m}$, via (1.8), we get the desired inequality:

$$
\tilde{\Lambda}(\mathrm{p})^{-1} \leq M^{\mathrm{p}} \Lambda(\mathrm{p})^{-1}
$$

\footnotetext{
${ }^{3}$ The difference quotient for these is 0.015014, and one more Shank transform yields 1.79651.
} 
Corollary 9.2. If $(\tilde{\mathcal{G}}, \tilde{\Gamma})$ and $(\mathcal{G}, \Gamma)$ are $M$-quasi-equal then

$$
M^{-\mathrm{p}} \tilde{\Lambda}(\mathrm{p}) \leq \Lambda(\mathrm{p}) \leq M^{\mathrm{p}} \tilde{\Lambda}(\mathrm{p}) \quad(\mathrm{p}>1) .
$$

Proof of Lemma 7.1. We fix $n \in \mathbf{N}$ and drop the subscript $n$, that is let $\mathcal{G}:=\mathcal{G}_{n}$ and $\tilde{\mathcal{G}}:=\tilde{\mathcal{G}}_{n}$. To align the input/output sets we rotate $\mathcal{G}$ by 90 degrees, so that the leftmost vertices are the inputs and the rightmost vertices are the outputs for both networks. For $\Gamma$ and $\tilde{\Gamma}$ we take the paths connecting input to output (as in (3.2)).

Because, by Proposition 3.1, $\tilde{\Lambda}(\mathrm{p})$ is comparable to $\tilde{R}_{n}(\mathrm{p})=\tilde{P}_{n}^{\mathrm{p}^{*} / \mathrm{p}}$ and $\Lambda(\mathrm{p})$ is comparable to $R_{n}(\mathrm{p})=P_{n}^{\mathrm{p}^{*} / \mathrm{p}}$, it suffices to show that $(\tilde{\mathcal{G}}, \tilde{\Gamma})$ and $(\mathcal{G}, \Gamma)$ are $M$-quasi equal (and then lean on Corollary 9.2).

Recall that, including the zero current edges along the sides for simplicity, $\mathcal{G}$ is the network carried by the tiling $\Phi_{\text {std }}^{n}\left(T_{A}\right)$ where $\Phi_{\text {std }}$ is as in Figure 6.14 and $\tilde{\mathcal{G}}$ is carried by the tiling $\Phi^{n}\left(T_{C}\right)$ where $\Phi$ is as in Figure 7.19. Although the networks they carry differ, the tiles for both tilings coincide and are the $3^{-n} \times 3^{-n}$ grid squares making up the carpet approximant $X_{n}$.

Given $\tilde{v} \in \tilde{\mathcal{V}}$ associate to it all the tiles that contain $\tilde{v}$ or intersect (touch) such tiles containing $\tilde{v}$. Define $B(\tilde{v})$ as the set of all vertices in $\mathcal{V}$ that belong to the tiles associated to $\tilde{v}$. We leave it as an exercise to verify the conditions (i),(ii), (iii) for a suitable $M>0$ (that does not depend on $n \in \mathbf{N}$ ). Thus $(\tilde{\mathcal{G}}, \tilde{\Gamma})$ is $M$-quasi contained in $(\mathcal{G}, \Gamma)$. The analogous construction with $\tilde{\mathcal{G}}$ and $\mathcal{G}$ switched shows the opposite $M$-quasi containment.

\section{References}

[1] Alamgir, M., and U. von Luxburg: Phase transition in the family of p-resistances. - Advances in Neural Information Processing Systems 24, 379-387, 2011.

[2] Albin, N. and F. Darabi Sahneh, M. Goering, and P. Poggi-Corradini: Modulus of families of walks on graphs. - In: Complex analysis and dynamical systems VII, Contemp. Math. 699, Amer. Math. Soc., Providence, RI, 2017, 35-55.

[3] Barlow, M. T.: Analysis on the Sierpiński carpet. - In: Analysis and geometry of metric measure spaces, CRM Proc. Lecture Notes 56, Amer. Math. Soc., Providence, RI, 2013, $27-53$.

[4] Barlow, M. T., and R. F. Bass: On the resistance of the Sierpiński carpet. - Proc. Roy. Soc. London Ser. A 431:1882, 1990, 345-360.

[5] Barlow, M. T., R. F. Bass, and J. D. Sherwood: Resistance and spectral dimension of Sierpiński carpets. - J. Phys. A 23:6, 1990, L253-L258.

[6] Bonk, M.: Quasiconformal geometry of fractals. - In: International Congress of Mathematicians. Vol. II, Eur. Math. Soc., Zürich, 2006, 1349-1373.

[7] Bonk, M., and B. KLEINER: Quasisymmetric parametrizations of two-dimensional metric spheres. - Invent. Math. 150:1, 2002, 127-183.

[8] Bonk, M., and B. Kleiner: Conformal dimension and Gromov hyperbolic groups with 2sphere boundary. Geom. Topol. 9, 2005, 219-246.

[9] Bourdon, M., and B. Kleiner: Combinatorial modulus, the combinatorial Loewner property, and Coxeter groups. - Groups Geom. Dyn. 7:1, 2013, 39-107.

[10] Bourdon, M., and B. Kleiner: Some applications of $\ell_{p}$-cohomology to boundaries of Gromov hyperbolic spaces. - Groups Geom. Dyn. 9:2, 2015, 435-478.

[11] Bühler, T., and M. Hein: Spectral clustering based on the graph p-Laplacian. - In: Proceedings of the 26th Annual International Conference on Machine Learning, ACM, 2009, 81-88.

[12] Cannon, J. W.: The combinatorial Riemann mapping theorem. - Acta Math. 173:2, 1994, $155-234$. 
[13] Carrasco Piaggio, M.: On the conformal gauge of a compact metric space. - Ann. Sci. Éc. Norm. Supér. (4) 46:3, 2013, 495-548.

[14] Clarkson, J. A.: Uniformly convex spaces. - Trans. Amer. Math. Soc. 40:3, 1936, 396-414.

[15] De Boor, C.: A practical guide to splines. Revised edition. - Springer, Berlin, Appl. Math. Sci., 2001.

[16] Elmoataz, A., M. Toutain, and D. Tenbrinck: On the $p$-Laplacian and $\infty$-Laplacian on graphs with applications in image and data processing. - SIAM J. Imaging Sci. 8:4, 2015, $2412-2451$.

[17] Haïssinsky, P.: Empilements de cercles et modules combinatoires. - Ann. Inst. Fourier (Grenoble) 59:6, 2009, 2175-2222.

[18] Heinonen, J.: Lectures on analysis on metric spaces. - Universitext, Springer-Verlag, New York, 2001.

[19] Herbster, M., and G. Lever: Predicting the labelling of a graph via minimum p-seminorm interpolation. - In: NIPS Workshop 2010: Networks Across Disciplines: Theory and Applications, 2009.

[20] Kapovich, M., and B. Kleiner: Hyperbolic groups with low-dimensional boundary. - Ann. Sci. École Norm. Sup. (4) 33:5, 2000, 647-669.

[21] Kigami, J.: Quasisymmetric modification of metrics on self-similar sets. - Geometry and analysis of fractals, Springer Proc. Math. Stat. 88, Springer, Heidelberg, 2014, 253-282.

[22] Kigami, J.: Weighted partition of a compact metrizable space, its hyperbolicity and Ahlfors regular conformal dimension. - arXiv e-prints, arXiv:1806.06558, 2018.

[23] LewIS, J.: Applications of boundary Harnack inequalities for $p$ harmonic functions and related topics. - In: Regularity estimates for nonlinear elliptic and parabolic problems, Lecture Notes in Math. 2045, Springer, Heidelberg, 2012, 1-72,

[24] Mackay, J. M., and J. T. Tyson: Conformal dimension. - Univ. Lecture Ser. 54, Amer. Math. Soc., Providence, RI, 2010.

[25] Mackay, J. M., J. T. Tyson, and K. Wildrick: Modulus and Poincaré inequalities on non-self-similar Sierpiński carpets. - Geom. Funct. Anal. 23:3, 2013, 985-1034.

[26] Malo, R. J.: Discrete extremal lengths of graph approximations of Sierpiński carpets. - Thesis (Ph.D.), Montana State University, ProQuest LLC, Ann Arbor, MI, 2015.

[27] Pansu, P.: Dimension conforme et sphère à l'infini des variétés à courbure négative. - Ann. Acad. Sci. Fenn. Ser. A I Math. 14:2, 1989, 177-212.

[28] Ramaswamy, S.: A simple proof of Clarkson's inequality. - Proc. Amer. Math. Soc. 68:2, 1978, 249-250.

[29] Schramm, O.: Square tilings with prescribed combinatorics. - Israel J. Math. 84:1-2, 1993, 97-118.

[30] Sierpiński, W.: Sur une courbe cantorienne qui contient une image biunivoque et continue de toute courbe donnée. - C. R. Acad. Sci. Paris 162, 1916, 629-632 (in French).

[31] Trson, J. T.: Sets of minimal Hausdorff dimension for quasiconformal maps. - Proc. Amer. Math. Soc. 128:11, 2000, 3361-3367.

Received 29 September 2017 • Accepted 8 August 2018 